\title{
Energetics of the human mind : an effort to show the neural correlates of mental efforts
}

Citation for published version (APA):

Otto, T. (2013). Energetics of the human mind : an effort to show the neural correlates of mental efforts. [Doctoral Thesis, Maastricht University]. Datawyse / Universitaire Pers Maastricht.

https://doi.org/10.26481/dis.20131115to

Document status and date:

Published: 01/01/2013

DOI:

$10.26481 /$ dis.20131115to

Document Version:

Publisher's PDF, also known as Version of record

\section{Please check the document version of this publication:}

- A submitted manuscript is the version of the article upon submission and before peer-review. There can be important differences between the submitted version and the official published version of record.

People interested in the research are advised to contact the author for the final version of the publication, or visit the DOI to the publisher's website.

- The final author version and the galley proof are versions of the publication after peer review.

- The final published version features the final layout of the paper including the volume, issue and page numbers.

Link to publication

\footnotetext{
General rights rights.

- You may freely distribute the URL identifying the publication in the public portal. please follow below link for the End User Agreement:

www.umlib.nl/taverne-license

Take down policy

If you believe that this document breaches copyright please contact us at:

repository@maastrichtuniversity.nl

providing details and we will investigate your claim.
}

Copyright and moral rights for the publications made accessible in the public portal are retained by the authors and/or other copyright owners and it is a condition of accessing publications that users recognise and abide by the legal requirements associated with these

- Users may download and print one copy of any publication from the public portal for the purpose of private study or research.

- You may not further distribute the material or use it for any profit-making activity or commercial gain

If the publication is distributed under the terms of Article $25 \mathrm{fa}$ of the Dutch Copyright Act, indicated by the "Taverne" license above, 


\section{Energetics of the human mind}

\section{An effort to show the neural correlates of mental effort}


(C) Copyright Tobias Otto, Maastricht 2013

ISBN 9789461592644

Production: Datawyse | Universitaire Pers Maastricht 


\title{
Energetics of the human mind
}

\section{An effort to show the neural correlates of mental effort}

\author{
DISSERTATION \\ to obtain the degree of Doctor at Maastricht University, \\ on the authority of the Rector Magnificus, Prof. Dr. L.L.G. Soete \\ in accordance with the decision of the Board of Deans, \\ to be defended in public \\ on Friday $15^{\text {th }}$ of November 2013 at 10.00 hours \\ by
}

Tobias Otto

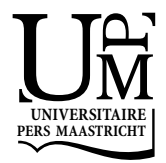




\section{Supervisors}

Prof. Dr. F.R.H. Zijlstra

Prof. Dr. R. Goebel

\section{Assessment Committee}

Prof. Dr. E. Formisano (chairman of the committee)

Prof. Dr. C.K.W. de Dreu, University of Amsterdam, the Netherlands Prof. Dr. B.M. Jansma

Prof. Dr. A. Riedl 


\section{Contents}

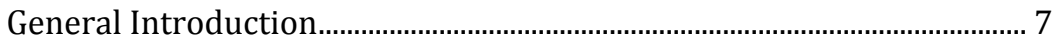

Chapter 1 History and development of the concept of mental effort .......................17

Chapter 2 Assessment of mental effort expenditure and its effects...........................27

Chapter 3 Making mental effort visible......................................................................... 41

Chapter 4 Neural correlates of mental effort evaluation - Involvement of structures related to self-awareness ...............................................................55

Chapter 5 Feeling the Force: Effort investment modulates activity in left anterior insular cortex

Chapter 6 Spatially distributed effects of mental exhaustion on RestingState fMRI networks: An ICA-based analysis of the resting human brain after sustained cognitive performance.

Chapter 7 Discussion 125

Summary 145

Samenvatting (Summary in Dutch) 149

Acknowledgements 153

Curriculum vitae 155 

General Introduction 
GENERAL INTRODUCTION 


\section{General Introduction}

I would like to begin by introducing the reader to the concept of mental effort. In common language, mental effort is often referred to as an abstract kind of "energy" which fuels our ability to perform, to concentrate, to put ourselves together to get something done. "Putting energy" into something is often used synonymously with "putting effort" into something. If we invest effort into something, for example when we prepare a presentation, we are motivated to achieve a good result. In turn, good results are also frequently attributed to a high amount of invested effort, next to more stable factors such as skill. If you imagine yourself for example making a presentation, every step of the process requires effort. You have to invest effort to bring the contents that you want to present up in your mind, you have to think about how you can structure these contents in a way that is suitable for your audience, and you have to actually make the presentation using for example a computer. Every step requires you to carefully process information, and later to transform this information into the output you would like to achieve. You invest effort into this by monitoring and regulating your mental processes, so that your mind works to achieve the desired goal. Effort is not only required for every individual part of this process, but also for general factors such as keeping your attention focused. Mental effort, put shortly, is thus the power that you deploy to regulate your mental processes in order to achieve a goal. Importantly, this regulation is different from the actual mental processes. The role of the mental processes is much alike the role of your muscles in a physical task: While your conscious mind invests the effort to regulate your movements, your muscles actually perform the task. In a mental task, you invest effort to coordinate your "mental muscles", or mental processes.

Investing mental effort is accompanied by a subjective feeling: While you are occupied with your task, you become aware that performing has an effect on you. The feeling might be present at the edge of your mind. Damasio, in his classical work on emotions and feelings, considered this class of "background feelings" to be a conscious readout of the representation of our momentary state (Damasio, 1999). The feeling of mental effort investment is thus related to the fact that you notice the effect that your state is affected by performing a task. You become aware that, to regulate your mental processes, you are investing a limited resource, and that investing this resource has an effect on your state. As you are regulating yourself, you command your mind to function in a way that is different from its momentary natural functioning. This is not to say that you are not motivated, or that you have an aversion against the task you are performing. If you have to invest effort into any task, it simply means that you have to divert your mind from its natural balance and temporarily devote it to achieving your goal. The inherent feeling that accompanies mental effort investment is your awareness of this diversion, paired with the knowledge from your experience that your power to keep your mind diverted is finite. 
Additionally, you are aware that your ability to use a particular mental process is limited, just as, to come back to our physical analogy, a muscle will get tired after sustained performance. To perform the same movement, you now have to invest more effort. The same goes for mental processes: After sustained performance, the same mental task requires more effort investment. At this point, however, we start to see discrepancies between physical and mental activity: While sustained physical activity prompts recovery in form of resting, recovery from sustained mental activity has been shown to benefit not from passive states, but rather from shifting the focus of activity (Rook \& Zijlstra, 2006; Zijlstra \& Sonnentag, 2006; Winwood, Bakker, \& Winefield, 2007). In the case that performance on a particular task must be sustained, though, the continuous use of a limited set of mental resources together with the continuous need to invest effort to steer your mind away from its idle state, will eventually result in the depletion of both the energy used to carry out mental processes and the mental effort used to mobilize this energy. As you thus feel how much effort you invest in a task, you feel how much power to regulate your mind and your mental processes is invested, and also how much energy is consumed by the performance itself. It is important to understand that investing mental effort is the act of mobilizing energy for and regulating its use by processing structures. The act of performance itself is thus separate from the act of investing effort, just as investing effort to lift a box is a mental act separate from the physical utilization of energy by the muscles.

This concept of a limited source that fuels your ability to regulate yourself is not new: as Baumeister et al. (2007) explain, the idea of "willpower" or "energy" as an underlying limited resource which enables a person to inhibit impulses is widely featured in folk literature. Psychology has adapted this view in form of the "strength model of self-control" (Baumeister, Heatherton, \& Tice, 1994; Baumeister et al., 2007). Baumeister et al. (1998) initially termed the process of spending a limited resource "ego depletion", a notion to the apparent limited amount of "active self", the part of the self-concept that actively works to actively steer one`s behavior in situations in which automatic responses might endanger long-term goals such as health.

While this strength model of self-control has on numerous occasions been shown to accurately describe the change of people's ability to regulate their impulses subsequently to facing challenging tasks (Baumeister et al., 1998); but see also Hagger, Wood, Stiff, \& Chatzisarantis, (2010) for a systematic review of the experimental literature), the model does not provide a hint at what this abstract, underlying and, above all, finite resource actually might be (Beedie \& Lane, 2011).

Especially the finite nature of mental effort has an important implication for any performance: At some point, we run out of mental effort to invest. Without the investment of a sufficient amount of mental effort, however, our mind is not devoted to performing the task. Errors start to happen, and at some point our behavior will be no longer helpful in pursuit of our goal. If we have the chance, we change our strategy, implying a pause in the pursuit of our goals at this point, recover, and 
then proceed. As individuals, we are generally able to estimate our "fuel" more or less accurately in relation to a task. Even more, we use our experience about how much effort we have to invest into something as a way to calculate the "costs" of an action in advance (Botvinick \& Rosen, 2008). Problems arise, however, when our estimations are incorrect: In the best case, we are not able to finish a paper in time for the deadline, in the worst case; we underestimate the amount of effort it takes to make a road trip and fall asleep at the wheel. Estimating someone else's need to invest mental effort is even more complicated: The terms "human error" and "largescale industrial accident" share such a close relationship partly as a result of the failure to accurately estimate the effort a person could or would invest into a task. Given both the universal relevance of mental effort in goal-oriented behavior and its implications for job design and safety, it is not surprising that especially occupational psychologists have aimed to define, model and measure mental effort.

The main problem in this lies in the abstract nature of mental effort: Although we do have a very specific feeling of strain that we associate with investing mental effort, this feeling remains a highly subjective experience. Even more, the associated processes happen in the mind, completely invisible to the outside world (Yeo \& Neal, 2004). This makes it very difficult to design a model of mental effort that incorporates all influential factors. The invisibility of mental processes and the subjective nature of the experience also complicate the measurement of mental effort: If we cannot measure it directly, and if we do not have objective correlates, how can we measure it at all?

If, by way of comparison, we take a look at the domain of physical effort, most people have a good idea of the factors that determine the amount of physical effort that needs to be invested in a task. Picture for example a person carrying boxes. In the first instance, we would assume that the muscle mass of the person and the weight of the boxes are the most influential factors determining the amount of physical effort that this person needs to invest. A certain amount of invested effort will result in a certain number of boxes handled in a certain amount of time. If we make the boxes heavier, the person will need to invest more effort to handle them. If the person is tired at the end of the day, he will also have to invest more effort to still handle the boxes, even without them being heavier. The reason for this increased need to invest effort lies in the state of the person himself: As the muscles grow tired and hurting from sustained performance, the natural preferred state of this person becomes a state of resting. To continue with the task, the person must now also overcome this tendency to disengage from the task, and ignore all additional de-motivating factors such as soreness or fatigue (see also Hockey, (1997)). Also, the general capacity of the person carrying out the task influences the amount of effort: A skinny person will have to invest more effort compared to the muscular person to carry the same box.

Our knowledge of the human body enables us not only to design a model of physical effort, but even enables us to measure objective correlates of physical effort investment such as increased pulse or breathing frequency. 
In the case of mental effort, such easy, straightforward predictions about the amount of effort needed by a person cannot be made from the outside. We cannot simply, like in a physical task, look at a person and see that this person is muscular or rather skinny; hence we do not have a straightforward estimate of someone's capacity.

Occupational psychologists have continuously attempted to overcome the elusiveness of mental effort and the framework of psychological factors that it is embedded in. In a wider sense, we try to understand how the capacity of a person is translated into utilizable resources, and how, given the right motivational incentives, a person uses these resources to perform any kind of task. What defines how long this person can perform? Which price does the person have to pay in terms of resources, and what does he or she gain?

Without the possibility to make the hidden processes of the mind and the brain visible, the strategy to investigate the nature of mental effort relied on designing progressively more elaborate models of it. The accuracy of these models could then be tested by experimentally evaluating if predictions derived from the models would correspond, for example, to measurable changes in performance, or in the experienced amount of mental effort that the operator could report. As we cannot simply measure obvious variables such as the pulse frequency to determine the amount of invested effort, we depend solely on the person him-or herself to give an account of his or her amount of invested effort, as all relevant processes happen invisibly in the mind and brain of the operator. Such self-reports of mental effort investment were quickly realized to be of great value, as the subjective account of effort investment by an operator was the closest approximation of the invisible mental process that, in the end, determined performance (Cooper \& Harper Jr, 1969). Efforts have thus been made to construct tools that enable operators to give comparable accounts of their effort investment.

Commonly used devices such as the Rating Scale Mental Effort (RSME; Zijlstra, 1993) or the NASA Task Load Index (NASA-TLX; Hart \& Staveland, 1988) measure the amount of subjectively felt mental effort in relation to a task, thus essentially asking the person who carried out a task to indicate the experienced level of subjective effort on a type of rating scale. These measurement devices assume thus that the acute experience of investing mental effort can be accessed at a later point in time to provide an estimation of that experience.

Our understanding of the process of self-evaluation of mental effort, though, is equally hampered as our understanding of mental effort in general by the hidden nature of the associated processes in the brain. We do not know how the brain of a person that is asked to provide a subjective account of his or her invested mental effort actually estimates this amount. Consequently, we also do not know if this mechanism is involved in the acute experience of mental effort investment.

At this point, however, by using methods from the field of neuroimaging, we are able to make the previously invisible processes related to mental effort and its evaluation by an operator visible. In our opinion, the best way to start this endeav- 
or is to use neuroimaging techniques to better understand the neural processes of self-evaluation of mental effort. Instead of attempting to grasp the nature of the abstract mental resource directly, we first addressed questions from the more defined area of measurements of mental effort. The crucial advantage in this approach is that it enables the use of well-studied instruments quantifying mental effort investment alongside neuroimaging techniques. By first testing the assumptions underlying this sort of instruments, we would learn more about how mental effort is represented in the brain. Equally important, by iteratively developing a paradigm in which we are able to manipulate mental effort investment and consequently its depletion, we would later be able to use these controlled manipulations to learn more about the nature of mental effort as an "energetic" resource.

In particular the specific issues around the underlying assumptions of selfreport tools can now be investigated by studying the brain during self-evaluation of mental effort. In order to contribute to provide a better insight, two questions in particular need to be answered, which form the first two research questions that I will discuss in this book:

1. Which neural mechanism underlies the subjective self-evaluation of mental effort investment?

2. Does the neural mechanism underlying self-evaluation of mental effort investment reflect acute, experienced changes in mental effort investment?

By answering these questions, we would gain evidence telling us if the self-rating tools to measure mental effort investment engage similar neural processes as the ones underlying the experience of mental effort investment. If the act of rating one's own effort investment would recruit the same structures as the ones that are sensitive to changes in required mental effort during performance, this would form an additional argument for the assumption that instruments like the RSME indeed measure the amount of effort that a person experiences. Identifying these neural structures does, however, also have some more fundamental implications: The knowledge about which brain structures play a role in self-evaluation of mental effort investment could tell also more about how the human brain can perform the complex task of evaluating an experience that originally happened in this very brain itself.

The neuroimaging tools that enable us to investigate these questions, however, offer possibilities as well to not only make the experience of mental effort investment visible, but to shed light on mental effort as an energetic resource as well. The characteristic feature of mental effort that inspired the analogy to an energetical resource is its finite nature. We cannot perform tasks endlessly, and performing an effortful task has a lasting effect. How is this reflected in the brain of a person that invested a substantial amount of effort? This forms the third research question that I aim to answer in this book. 
3. How does the investment of a substantial amount of mental effort affect brain functioning?

The importance of understanding mental effort as a finite, energetic resource cannot be overstated; as the universal importance of this resource for human functioning makes it a relevant factor in nearly all areas of life that depend on mental performance. The depletion of this resource signals that a profound change has taken place in the individual. This manifests overtly, in the behavior of the operator, as a lack of motivation to sustain performance any longer by investing excessive amounts of mental effort, inevitably leading to disengagement from the task (Meijman \& Mulder, 1998). Given sufficient investment of self-regulatory activity on previous tasks, even performance on subsequent different tasks necessitating self-regulation are impaired, even if those tasks require different sorts of information processing than the previous ones (Baumeister et al., 1998). This overt manifestation, however, can be assumed to be the result of profound covert changes in the brain of the operator that take place as a result of sustained effort investment. It is not known how exactly these changes in brain function manifest themselves, or which neural structures are affected. Showing how the depletion of this resource affects brain functioning would in particular be a potential first step to develop ways to objectively measure changes in the amount of effort a person has at his or her disposal. In a wider sense, gaining a more complete picture of how mental effort investment is implemented in the brain would make it possible to understand how mental effort as a concept describes the functioning of the brain. In the end, any kind of performance refers to more or less specialized mental activities, which can be linked to specific neural structures. The concept of mental effort seems to describe a functional principle of the brain that is beyond the single task: Mental effort is both required and consumed by every kind of mental performance alike.

\section{Outline of this book}

The aim of this book is to demonstrate how the concepts and instruments concerned with mental effort, as they are used and devised in occupational psychology, have a close relation to specific neural structures and their functioning. Hence, the main part of this book consists of the description and discussion of three experimental studies which we conducted to gain a better insight into the neural processes related to mental effort and its self-evaluation by operators. In the first three chapters, I will give a more detailed account of the theoretical background and the motivation behind this project.

In chapter 1 , I will provide a condensed overview of the development that lead to modern models of mental effort. This process reflects an interesting change in 
the view of the person that invests mental effort: While the person was seen as an almost mechanical, automatic processor in the earlier models, each subsequent model provided more conceptual autonomy to the person, recognizing the ability of humans to interact with their tasks in a flexible way. Especially the integrated model of mental effort by Mulder (1986) is presented in detail, as it forms the conceptual framework on which this work is based.

Chapter 2 focuses on the different approaches that are or have been used to measure mental effort investment. The purpose of this is to give the reader an idea of the multitude of attempts that have been made to find the ideal way to measure mental effort investment, both with cognitive instruments and in physiological correlates. A comparison of different approaches and techniques is made, which demonstrates that some measurements of mental effort investment have crucial advantages over other approaches.

In chapter 3, I describe how the hypotheses that I have derived from the literature can be investigated using neuroimaging methods. The chapter cumulates in the formulation of specific research questions and hypotheses. These first three chapters are intended not only to illustrate the motivation for and the theoretical use of this project, but also to give readers a brief introduction into the concept of mental effort. The experiments that are described in the later chapters are designed with the theoretical framework which encompasses mental effort in mind. Approaching this project from an occupational psychology perspective made it possible to use neuroimaging methods to investigate specific questions originating from the framework of mental effort and to design these experiments to make use of the theoretical possibilities that are offered by this framework. This aspect sets this work apart from earlier neuroimaging studies that utilized isolated aspects of mental effort, an approach which is fit to investigate the affected neural structures, but which does not add to our understanding of mental effort as such.

Over the course of chapters 4, 5 and 6, I present three studies that I have conducted to solve the research questions that I have formulated in chapter 3 . Described in chapter 4 , the first study focused on identifying the neural correlates of the self-evaluation of mental effort investment on a working memory task. In chapter 5, I present a study extending this design in the sense that it included multiple factors influencing the amount of mental effort the participants had to invest. In this second study, I investigated which parts of the brain responded to changing levels of mental effort investment during task performance. This made it possible to compare the neural correlates of effort experience to the earlier identified correlates of effort self-evaluation.

Chapter 6 extends the investigation of acute neural correlates of effortful performance by taking a look at the effects that sustained investment of mental effort has on the functioning of the human brain. The question of how brain functioning is altered by of sustained effort investment is one of the most fundamental questions that occupational psychology must solve. The study that is described in this chapter 
is the first attempt to relate the effect of sustained effort to make these changes visible.

In chapter 7, I will summarize the findings of all three studies and integrate them into the general scope of this book. The results yielded by the experiments seem on first glance to provide mainly an extension of our knowledge about which neural structures are affected by investment and experience of mental effort. The true value of these insights becomes only clear, however, when they are related back to the theoretical framework of mental effort. At this point it becomes clear just how closely related the concept of mental effort is to the functional principles of the human brain. This close relation has a number of implications in both theoretical and applied directions, some touching our concept of effort as an energetical resource, others prompting an extension of the scope of occupational psychology's overarching goal of improving work to also regard the neurobiological effects that work has on an operator. This chapter closes with an outlook of the promising combination of the fields of occupational psychology and neuroscience. 
Chapter 1

History and development of the concept of mental effort 
CHAPTER 1 
The concept of mental effort as it is used today evolved in several characteristic steps: initially, when looking at a person in the act of performing a task (to whom, for the sake of brevity, we shall refer to as "operator"), the task itself stood at the focus of attention. Although this approach did not explicitly include the concept of an energetic resource, it implicitly included the notion that humans have a limited capacity at their disposal, and that varying the nature of a task (the "task load") affects the associated processing costs for an operator. Yet, all factors that were seen as influential for the outcome in terms of performance were mainly sought for in the task. The operator was merely seen as an almost mechanic processor, which would act the same under all circumstances. Although this assumption seemed true for the very basic tasks that it was tested on initially, further research revealed severe shortcomings, hence fostering the development models that allowed for a more interactive role of the operator in relation to the task.

\subsection{Early observations of limited capacity}

The first step toward the concept of mental effort as it is employed today was the observation that humans possess a limited capacity to process information. Early models that try to account for this fact date back to the $19^{\text {th }}$ century, when the Dutch professor F.C. Donders introduced the idea of mental chronometry. This idea paved the road to his now-famous subtractive method (Sartori \& Umiltà, 2000). The basic principle behind his idea was that, compared to the reaction time on a baseline task, an identical task with an added subtask would lead to a longer reaction time. For example, if an operator was tasked with adding up several numbers that are simultaneously presented on a screen, this task would take a certain amount of time. An added subtask, as counting how many numbers are odd or even, would require extra processing time; hence the operator would need longer to perform the whole task. The difference between the two reaction times would be attributed to the processing time consumed by the added subtask. By making this assumption, Donders' model was one of the first models recognizing the fact that the human information processing system is indeed limited in its capacity. Yet, this early view still treated the nature of information processing in a rigid and mechanistic fashion. The limited processing capacity was assumed to be allocated equally over all processes that the task requires, such as reading the numbers, storing numbers in working memory, or calculating the sum.

The first major conceptual improvement to this somewhat rigid view was provided by Moray (1967). Instead of assuming that an operator mechanically carries out a task in a fixed series of processing stages, Moray drew an analogy to the emerging developments in computer science: In his view, the single stages of processing could be seen analogue to specialized computer programs that each perform a specific operation on the data that is given to them. The total processing 
capacity of the human information processing system was still seen as fixed and stable. However, Moray included an additional "executive" program that could strategically allocate the processing capacity to the different programs. Thereby, Moray`s framework was the first one that included the concept of an operator who had the capability of strategically managing his or her limited processing resources instead of performing every part of a task in a rigid fashion.

\subsection{Energetical metaphors}

Using the conceptual improvements of Moray, Kahneman (1973) went a step further by replacing the different serial stages of processing with different processes, which allowed accounting for parallel processing. Kahneman also added a key concept to the processing oriented capacity approach in form of the metaphor of an underlying, limited energy source which plays a crucial role in fuelling processing. He identified this limited resource as arousal, the general psycho-physiological level of activity (see Figure 1). In this model, arousal formed the hierarchically highest parameter in the regulation of processing. An ideal level of arousal would lead to an optimal capacity for task performance. Capacity in this model is used synonymously with effort or attention that is invested in a task. Most important, capacity was only seen as influential for processing itself. Only the occurrence of errors due to a lack of capacity would, triggered by the evaluative feedback mechanism, cause an increase in arousal. Any failure to up-regulate arousal in the face of insufficient performance is attributed to motivational factors or failure of the feedback system. A direct, willful regulation of arousal is not part of the model. Too much arousal, thus, is attributed to factors such as anxiety, which in turn cause a dysfunctional over-focusing of the information processing system. 


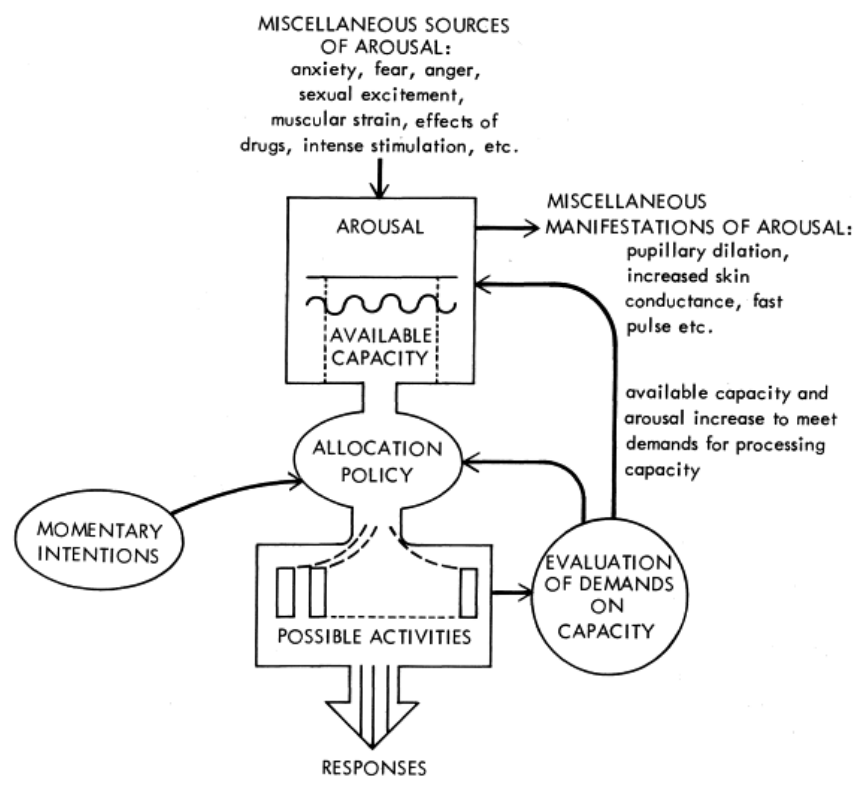

Fig. 1: The capacity model of attention and mental effort (from Kahneman, 1973).

The introduction of arousal as well as a limited capacity in form of a resource was an explicit recognition of the fact that the operator could be influenced by maladjusted levels of arousal. Although this fact had been stated already in the beginning of the $20^{\text {th }}$ century in form of the Yerkes-Dobson law, Kahneman's model was novel in emphasizing the role of arousal as a central factor that also had particular effects on information processing. Obviously, under real-world conditions, interfering factors such as fatigue, but also sudden distractions, sickness or drugs can interfere with the state of the operator. By integrating arousal as the limited and necessary source of energy into his model, Kahneman established a more accurate view of the operator. As noted by Hockey (1997), Kahneman was also first in actually separating the concepts of arousal as generalized energy and employed energy in the form of capacity, or processing effort. In this model, effort was seen as the mobilized form of energy that is used to regulate information processing. In summary, according to Kahneman`s model, effort only played a role as a regulative resource which requirements is determined solely by the task demands.

\subsection{Two distinct approaches}

Kahneman's view of mental effort as employed energy can be seen as the first part of the modern mental effort construct. Still, in this view, the amount of mental effort that an operator needs to invest for successful task performance was determined solely by the task load. This view of mental effort being analogous to processing 
demands has been called the "processing effort" approach. The processing effort approach still focused mainly on the task-sided depletion of resources, thereby locating the main part of the workload in the computational demands of the task itself. Yet, as Kahneman (1973) already stated himself, the amount of available energy in the form of arousal depends on the psycho-physiological state of the operator.

Broadbent (1971), as cited in Mulder (1986), pictured arousal regulation as the most important regulative mechanism that can influence the actual state of an operator. Kahneman had already integrated arousal as a central part of his model. Broadbent adapted the role of arousal: arousal was still the direct fuel of information processing, yet the role of effort was radically changed: mental effort was now seen as a more universal regulative resource. In addition to enabling control of information processing, Broadbent pictured the regulative abilities of effort to extend also to the adjustment of arousal. For this, Broadbent devised two mechanisms that determine the level of arousal. One generally responsible lower mechanism, influenced by stressors such as noise or intoxication, would determine the level of arousal automatically. A higher, incidental mechanism, influenced by acute conscious devotion towards a purpose, could be used to consciously regulate one's levels of arousal. This might be necessary in cases were one is to tired (low arousal), or too anxious (high arousal) to concentrate on a task. In such a case of a maladjusted level of arousal, the higher mechanism can override the lower one to secure a level of arousal that optimizes performance under the given circumstances. This regulative action comes at the cost of effort.

The consideration of an operator who has to invest effort in order to reach and maintain a state that allows sufficient performance in a task is known as "state approach" (Zijlstra, 1993). This approach forms a complimentary view to the processing effort approach, as it allows the (effortful) regulation of the operator's arousal level. This allowed for a conceptual inclusion of dynamically changing states of the operator, as they would occur in real-world scenarios for example in the case of fatigue. The need for regulative action to adjust levels of arousal, as caused by a suboptimal psycho-physiological state of the operator, is referred to as the state load.

It becomes clear at this point that workload cannot be determined by focusing on the demands of a task (the task load) alone, as it was done in the earlier models. As the state of an operator changes, this does not automatically change the performance of the operator. Instead, the operator is faced with the need for additional regulation in the form of the state load. Only the combination of state and task load forms a meaningful concept of the total workload that needs to be met by the investment of mental effort. 


\subsection{Development of a unified concept of Mental Effort}

A conceptual unification of the processing approach and the state approach was first brought forward by Sanders (1983). He employed a staged processing view with four different stages of processing: Stimulus preprocessing, Feature extraction, response choice and response evaluation. In Sanders' model those processing stages were fueled by three types of resources: Arousal, activation, and effort. The new key elements of his model were first of all the existence of an evaluative mechanism, which signaled the need for adjustment in one or more domains. Second, arousal and activation were considered to be energetical resources linked to specific functions or stages. The role of effort was split and extended: It was seen as both necessary for the conscious, non-automatic processing of information and for the adjustment of arousal and activation.

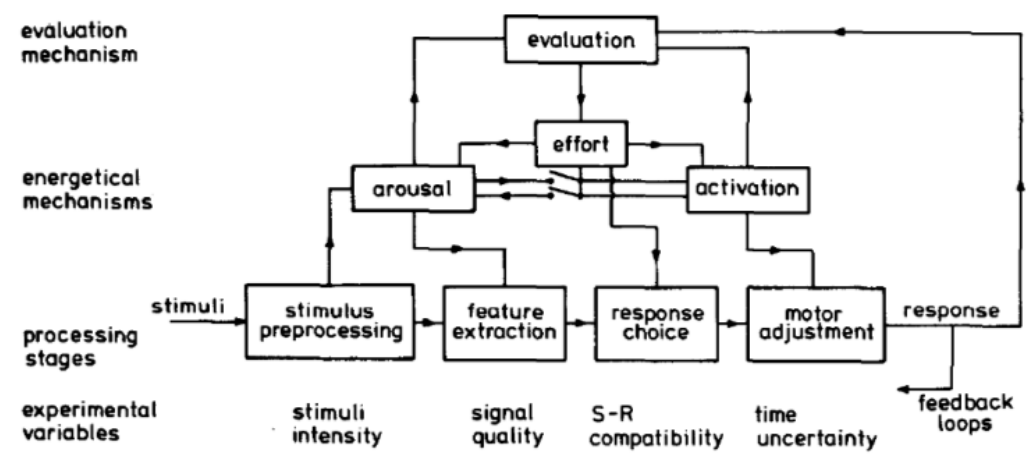

Fig. 2: Sanders' model of mental effort was the first attempt to combine the dual roles of effort as regulating both the processing of information and the level of arousal. Effort was pictured to influence the response processing stage, as the earlier stages of processing are not open to conscious intervention. Activation, also subject to effortful regulation, refers to the readiness to perform motor responses or similar output. (from Sanders, 1983)

Mulder (1980) further developed this concept into his "integrated model of mental effort" (see Figure 3). Mulder continued to use Sander's three separate energetical resources, now related distinctively to input (arousal), output (activation) and controlled executive function (effort). In his model, effort is simply required for any activity which involves controlled processing or adjustment, either during the conscious processing of information or due to regulation of the two other energetical resources. The origin of the need for effort can be internal or external, but controlled executive functions are treated as equally tapping on the same specific resource.

In the integrated model of mental effort, effort fuels both the regulation of processing and the regulation of arousal, similar to the model of Sanders (1983). The integrated model of mental effort, however, emphasizes the role of effort as a compensating resource. Mulder`s model furthermore permits an indirect regulation of 
other processing stages via the central processing module, accounting for top-down control. Such top-down control takes place for example when modules at an earlier part of the sensory processing chain are "instructed" to be especially sensitive to a certain type of stimulus (think about playing who sees the most VW Beetles as a child) (Shulman et al., 1997). Mulder also integrated a basic reservoir underlying effort in form of the overall activation of the brain as controlled by the reticular formation, thereby incorporating the dependency of cortical functions on the appropriate basic state of the individual.

The integrated model of mental effort makes it possible to account for the complex interaction of operator and task, and for the outcomes in terms of performance. As stated earlier, the total workload is determined by the task load as well as by state load. This means that even despite a constant task load, the total workload can increase when for example the state of the operator deteriorates due to sustained performance. This is not, however, immediately reflected in a drop in performance: As long as the effort reservoir is able to provide additional mental effort, the operator can choose to invest this extra effort to maintain the performance level. In this case, two things are bound to happen: On the one hand, the prolonged sustained performance will lead to a further increase in state load and thereby to an increased requirement for mental effort investment; on the other hand, the available amount of mental effort will be further drained. Note that the only obvious output, the task performance, remains unchanged, while the amount of invested effort increases. In the end, the operator can either change the performance goals to decrease the task load, disengage completely from the task to recharge the effort reservoir, or risk a possibly sudden drop in performance when effort runs out.

By permitting a flexible role of effort in both the regulation of processing and state, the integrated model of mental effort is able to account for real-life scenarios better than models which only focus on task-sided factors and performance as an outcome. Even more, the integrated model also allows making predictions about the subjective experience and the behavior of the operator. As the earlier models were focused on the task and took a more engineering-based approach, the integrated model does not treat the operator as a fixed, mechanistic factor, but as an autonomous agent in a complex interaction. 


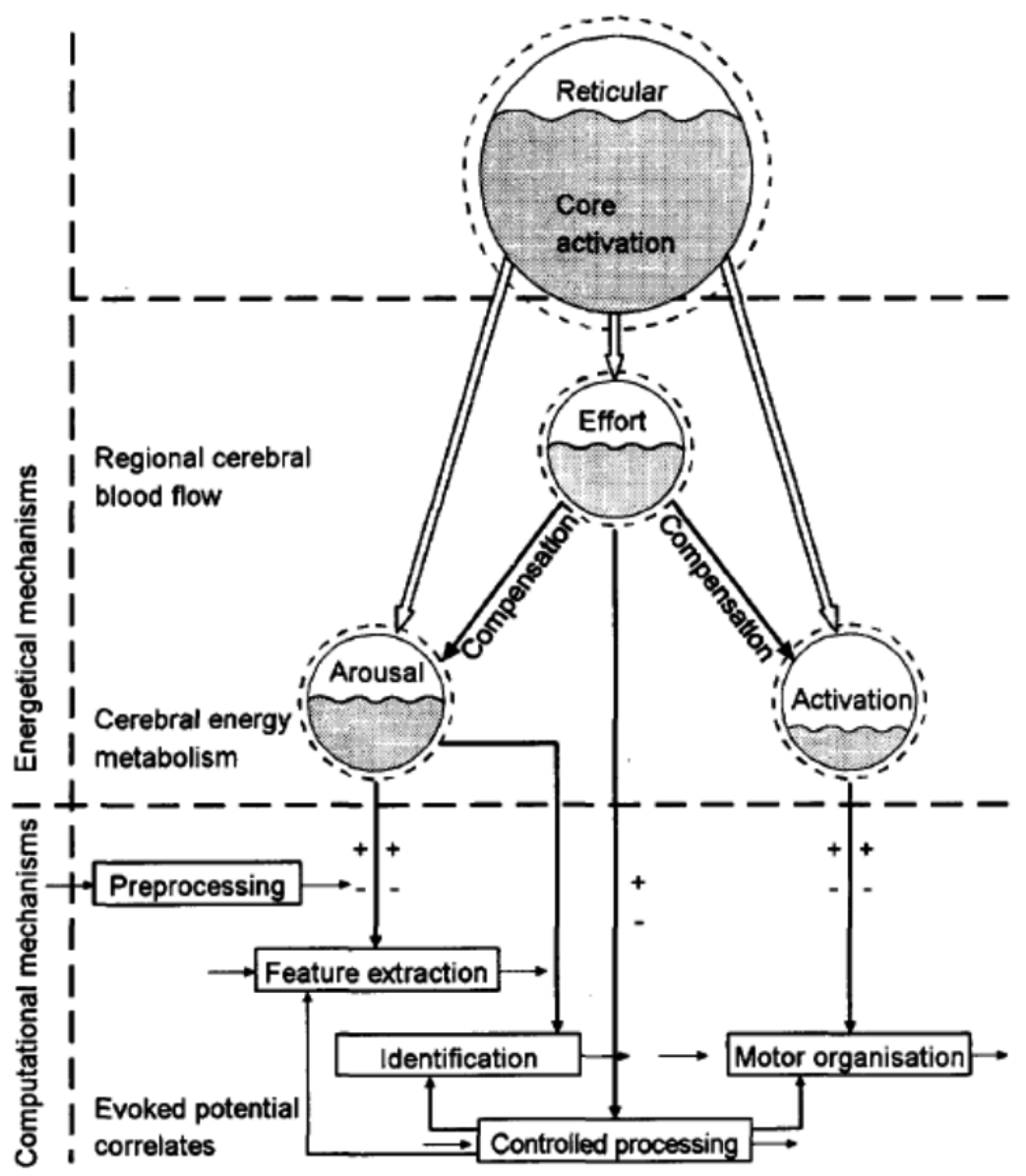

Fig. 3: The integrated model of mental effort (from Mulder, 1986)

The integrated model of mental effort provides a comprehensive account of the factors present in a task-operator relationship that define the amount of mental effort that needs to be invested to meet the mental workload. Effort is seen as the resource that drives all regulative processes, no matter if they concern processing, attentional control or regulation of the state of the operator. More recently, this conceptualization of a single resource underlying all regulative processes has become a central feature in a prominent model of self-control impairments, the strength model of self-control (Baumeister et al., 1994; Baumeister et al., 2007). Central to this model is the notion that investment of self-control, meaning the active regulation of one`s thoughts and behaviors, consumes a limited resource. Hence, on subsequent tasks requiring self-control, the ability of subjects to effectively exert self-regulation is reduced, increasing the probability of a failure to, for example, inhibit an undesirable behavior. Evaluation of this model by means of experimental studies has consistently produced corroborating evidence (Hagger et 
al., 2010). This shared assumption of a limited resource underlying self-regulation closely links the strength model of self-control and the integrated model of mental effort very closely together. Yet, while this resource, in the form of mental effort, is modeled more in its relation to regulative and cognitive processes in the integrated model of mental effort (Mulder, 1986), it remains more obscure in the strength model of self-control.

The concept of mental effort as it is used today is the product of an iterative process. Starting with the notion of a limited processing capacity of an individual, the focus shifted towards the factors that influence the ability of the individual to perform tasks. As a result, the individual itself and its cognitive processes became the subject of interest. This, in turn, provided the foundation for the construction of models that incorporate the interaction of an individual with a task in an interactive manner. As I will elaborate in Chapter 3, this level of refinement has reached a point in which the current models converge on a conceptualization of mental effort as a coherent resource underlying self-regulation. As a result, the question of the nature of this resource poses itself as the next logical research goal, but, at the same time, constitutes a methodological challenge: In order to improve our understanding of this abstract resource, we have to integrate approaches beyond the traditional cognitive experimental setups, as these seem to merely confirm the current models at this stage, not adding fundamentally new insights. Our chosen approach to use neuroimaging methods to gain knowledge about the neural correlates of mental effort in this context relies on the utilization of the known features of mental effort to develop a research line that extends our knowledge related to the present concept of mental effort. Without a very close integration of the concept of mental effort in our research designs, we would merely create data that would, at best, yield questionable relevance for said concept. Our approach thus initially focusses on extending our understanding of the quantification of mental effort, as these provide explicit indicators of changing levels of effort investment in operators as a result of controlled manipulations of the factors that have been shown to influence it.

The view of effort as the single underlying resource has an important implication for any tool that aims to measure mental effort investment according to this rationale. It would not be sufficient to just measure the effort that is needed for example for the regulation of processing. A measurement device that aims to measure mental effort investment according to the integrated model of mental effort needs to measure the total combined amount of invested effort. In the next chapter, I will describe a number of measurement devices and techniques which have been designed with this goal in mind. As approaches to measure mental effort also includes investigations of the usefulness of indicators of neural activity for this purpose, I will also discuss how these approaches so far are related to the wider body of neuroimaging research that has used, alas mostly limited, experimental manipulations of mental effort. 
Chapter 2

Assessment of mental effort expenditure and its effects 
CHAPTER 2 
In this chapter, I will elaborate on why it is important to be able to measure the amount of invested mental effort. I will also describe some ways used to measure mental effort investment in relation to a task. Previous research into measurements of mental effort investment was conducted mainly before neuroimaging methods were widely available, hence it was not possible to investigate the neural foundations of mental effort expenditure, its experience by an operator and the consequences that this expenditure has on brain functioning. The concept of mental effort clearly does refer to the neurobiological basis of information processing in the brain. However, the understanding of the neural correlates of mental effort was, at the time, limited to the regulation of arousal by the brain stem (Mulder, 1986) or the effort-related peripheral reactions and regulations by the autonomous nervous system and the neuroendocrine system (Meijman, 1989).

Most neuroimaging research that touches mental effort, on the other hand, is limited by only employing the concept of mental effort in an incomplete fashion, e.g. only employing variations in task load throughout an experimental paradigm. As described by Mulder (1986) in the integrated model of mental effort, task load is only one factor that determines the total amount of mental workload. State load, the component of the total workload that results from the need to regulate one`s own state to perform a task, forms a substantial determinant of the total amount of mental effort required for successful performance. Most important, this factor is not directly related to the requirements of the task itself: Even the act of performing a simple task, in itself requiring moderate amounts of effort investment, can require disproportionate amounts of effort if the operator is in a state that is not suitable for performance.

Thus, neuroimaging research that only employs variations in task load neglects the second influential component of the total workload. This entails two consequences: First, it can be difficult or impossible to separate neural changes mediated by changes in effort investment from neural changes mediated by variations in task complexity or processing load. Second, the state load component of the mental effort construct refers to the occurrence of varying states or dispositions of the very information processing system that needs to perform the mental task. Without including controlled manipulations of state load in experimental paradigms, this important aspect of mental effort in particular, but also of the human information processing system in general would remain obscure, no matter how advanced the research tools are.

\subsubsection{Development of measuring devices for mental effort}

As illustrated in the integrated model of mental effort, a great number of factors influence the amount of effort that an operator needs to invest to perform a task. All factors that influence the amount of required mental effort must be taken into account, as the performance of the operator is only warranted if the need of effort investment does not exceed the amount of effort the operator is willing or able to 
invest into the task. This makes the question of how much effort a task will require from an operator quite non-trivial. However, being able to estimate the amount of mental effort that operators need to invest to deliver sufficient task performance is crucial in a lot of situations, most prominently in the design of work places and interfaces. Especially in work environments that rely on uncompromised operator performance, it must be assured that the total workload stays within the capacity of the operator, and that this is ensured also at the end of the shift.

An example of such an environment can be found in the aviation industry. 0perating an aircraft is a highly complex process, and the consequences of an operator not being able to invest the required amount of effort for this task are potentially severe. If we are thus interested in the amount of mental effort a pilot has to invest into the task of flying a particular airplane, it is not sufficient to measure the amount of mental effort that is needed to perform the single parts of the larger task separately. We could, of course, measure how much mental effort a pilot needs to invest for the subtask of keeping the plane on a certain course and altitude. We could also measure how much mental effort other subtasks as checking the fuel level require. In a complex work environment, however, subtasks can interact with each other in an unpredictable way. Checking fuel levels during flight, while keeping a plane on course, might initially not exceed the amount of effort that a pilot can invest at any moment. If, however, keeping the plane on course requires more effort due to bad weather conditions, the need to additionally interact with the fuel management system could increase the total amount of effort the operator needs to invest to a level that the operator is not able to sustain. Even more, the attentional capacity of the pilot might be overloaded, a case that is beyond any remedy by further increase of effort investment. Also, a pilot has not only to be able to momentarily sustain the required amount of mental effort. Performance must be secured for the total duration of his shift, and reserves of effort must be kept for critical phases as landing the plane. While factors as stormy weather or the duration of a flight are likely to be regarded in the design phase of the aircraft, other situations where subtasks interact in a dangerous way might not be as predictable. As a result, it might be impossible to predict the feasibility of a work interface without actually testing the operator-task interaction under the aspect of mental effort expenditure (Cooper \& Harper Jr, 1969).

There are several different approaches to measure or estimate the amount of mental effort that an operator needs to invest into a task. Subjective measurement devices require the operator to rate his or her own invested mental effort on one or several scales after performing a task. Other methods try to derive an estimation of the amount of mental effort from physiological correlates during performance. These methods have been tested under various circumstances, and the results of these tests have led to a number of questions. 


\subsubsection{Subjective ratings of mental effort}

Historically, there have been different approaches to measure the subjective experience of mental effort investment. These approaches can be divided into multi and one-dimensional approaches. Multidimensional approaches follow the reasoning that perceived workload (and thus the amount of effort required to meet it) is inherently influenced by different factors, for example the processing complexity, the time pressure under which the task has to be performed, but also if there is the need for the operator to modulate his or her own state, for example in terms of arousal. Multidimensional scales aim to measure all of these influential factors by assessing them separately.

Two well-known examples of scales that are designed in this way are the Subjective Workload Assessment Technique (SWAT; Reid, Eggemeier, \& Shingledecker, 1982) and the NASA Task load index (NASA-TLX; Hart \& Staveland, 1988). The SWAT distinguishes three different dimensions: time pressure (determining possibilities for parallel processing or task overlap), mental effort (in this case defined as processing capacity consumption) and stress load (the amount of frustration, confusion or anxiety elicited by the task) (O’Donnell \& Eggemeier, 1986).

The NASA-TLX is composed of six different dimensions: Mental task demands, physical task demands, time pressure, and success of the performance, effort and frustration level. Additionally, operators have to weigh the contribution of each dimension to the overall experienced workload. The NASA-TLX was not exclusively tailored for the use in mental tasks, but for workload assessment in general, covering all relevant factors. Both the SWAT and the NASA-TLX, but also multidimensional subjective workload assessment scales in general have drawn some critical comments on them. First of all, multidimensionality refers to certain aspects of the subjective experience of the operator. It is highly questionable if these aspects are reliably defined in the same way across operators. It depends furthermore on the task and on the interpretation of the operator if these dimensions encompass the full spectrum of the subjective experience of workload. Additionally, the single factors have been shown to be highly correlated (Zijlstra, 1993).

The first application of a simple one-dimensional rating scale was the CooperHarper-Scale, a simple ten point scale on which pilots could rate the "flyability" of an aircraft. This scale is a very simple way to investigate the mental workload posed on an operator by a plane, evaluating the ratings of different operators. The subjective rating is important, as especially in an airplane the flight control task demands are supplemented by a vast number of additional stressors that are very difficult to foresee (Cooper \& Harper Jr, 1969). Interestingly, in their more application-focused study, Cooper and Harper actually describe the implications that only later became the foundation of Mulder's integrated concept of mental effort. In the field of aviation psychology it is important to assess if pilot and vehicle interact in a way that makes the combination suitable for fulfilling a goal. Knowing the task demands alone is not sufficient to predict the sustainability of the pilot-vehicle- 
interaction. The Cooper-Harper scale is only of limited value for other research, though, as it is tailored for the use in the aviation industry and associated research (Zijlstra, 1993).

A later approach to establish a one-dimensional scale was made by Borg (1972), who developed a rating scale with verbal descriptions of different levels of task difficulty. The verbal descriptions were used as anchor points on a scale, which helped operators to orient themselves in terms of the magnitude of the variable. Although these scales have been employed with great success, the method of choosing the anchor points and their distances has been the main source of critique for the constructed scales, especially for the one developed by Borg. As Zijlstra (1993) arguments, the employed adjectives such as "average" might rationally indicate a medium level, but are often used ambiguously in common language. Zijlstra therefore went on to construct his own scale, the Rating Scale Mental Effort (RSME). In this rating scale, he employed a total of nine anchor point items which were extensively evaluated for their common interpretation in terms of related levels of effort investment. The second improvement was the spacing of the anchor points according to the ratings of operators: The distances between the actual anchor points were not equal, but reflected the intuitive ratings of the single items given by groups of subjects. The result, shown in figure 4, was a one-dimensional scale ranging from $0-150$, on which the anchor points were placed between zero and 150, but not too close to those values ( 2 and 114, respectively). The ratio behind the latter design choice was that the numerical values do not represent absolute values, but rather relative differences between levels of invested mental effort. The RSME has been validated using both manipulations of task- and of state load (Zijlstra, 1993). In direct comparisons, it was found to be more sensitive in estimating mental effort than the NASA-TLX (Veltman \& Gaillard, 1996) and was found to be both more sensitive and less complex than the SWAT (Verwey \& Veltman, 1996). The evidence is thus in favor of a rating scale that asks the operator to provide one coherent estimation of invested mental effort, as opposed to instruments that try to separately measure different aspects of the experience that might be influenced by either taskor state load - related factors.

The validation research of the RSME thus provided evidence that shows that the ratings provided by the operators are indeed influenced by factors from both the domain of task load and state load. 


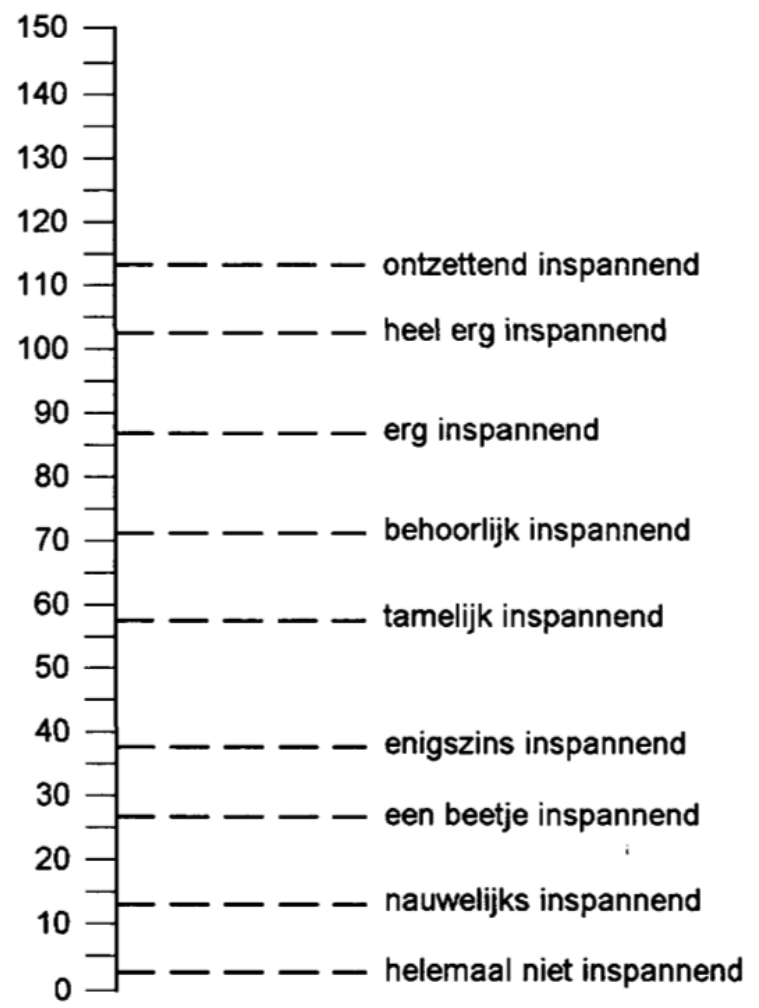

Fig. 4: the Rating Scale Mental Effort (RSME; from Zijlstra, 1993)

Nevertheless, the RSME and all other subjective scales work with the assumption that the estimations provided by an operator after the act of performing a task reflect the averaged amount of mental effort investment that the operator experienced during task performance. To test this assumption, it would take a different measure of mental effort that could be taken during task performance. Ideally, such a variable would not rely on the operator providing a similar kind of subjective selfevaluation, and it should be possible to measure it without the measurement causing changing levels of interference with the operator`s task. Previous studies have attempted to locate such a variable among the large number of physiological variables that can be measured in an operator.

\subsection{Physiological measurement methods}

Physiological variables offer the advantage of mostly being easily accessible without interfering with the operator's performance on a task. Most of these variables, however, suffer from various shortcomings which prevent them from providing an estimate of the total amount of invested effort. Nevertheless, the accumulated evi- 
dence from previous research provides a direction for a different approach that I will discuss.

Physiological variables that have been researched include, among others: pupil dilation, eye scanning randomness, visual fixation frequency, muscle tension, blink rate, respiratory rate, adrenaline level, blood glucose levels and various cardiac variables. While these variables represent quite indirect correlates of acute mental effort investment that are measured in the periphery of the body, attempts have also been made to measure correlates of mental effort using measurements of brain activity. Comparative reviews of the validity of these variables as indicators of mental effort investment have been conducted previously, thus interested readers are referred for example to Veltmann and Gaillard (1996), Brookhuis and de Waard (2010) or to the extensive review by Staal (2004). I will first describe a cardiac variable that has been researched extensively, and which also has been compared directly with the RSME as a subjective measurement device.

\subsubsection{Cardiovascular effects of Mental Effort}

The connection between mental effort and changes either in cardiac variables such as blood pressure or heart rate is believed to be mediated by the autonomic nerve system. The autonomic nerve system is tasked with configuring the various systems of the body so that physiological requirements for task execution can be met. For instance, the heart rate will increase during physical activity to ensure a sufficient supply of oxygen. Next to well-known parameters such as the heart rate or blood pressure, there are also other parameters of cardiac functioning that form potential indicators of effort investment. Mulder (1980) focused his attention on the socalled $0.1 \mathrm{~Hz}$ component of heart rate variability. This component can be understood as a measurement of the regularity of the heart rate. The heart rate of a human is not completely regular, but varies slightly. Most of the variation happens in the frequency band of $0.1 \mathrm{~Hz}$, thus comparing periods of ten seconds with each other yields prominent differences in heart rate. These differences are mediated by the system that serves to regulate the blood pressure according to the physiological needs of the body. This system is called the baroreceptor blood pressure control system. The baroreceptors are pressure sensors located in the larger arteries. By varying blood vessel constriction, heart rate and heart constriction power, this system serves to regulate the blood pressure. The control of the different variables is mediated via the autonomous nerve system. Mulder (1980) conducted one of the first attempts to utilize the observation that this $0.1 \mathrm{~Hz}$ component is greatly reduced by mental workload. He explained this reduction in variability with a decreased sensitivity of the brainstem for the impulses of the baroreceptors during an effortful state. In particular, the $0.1 \mathrm{~Hz}$ component of the HRV was found to correlate with a person consciously facing a threat to mental equilibrium. Interestingly, it was found that this can be both a difficult task on which the subject is willing to perform or also a threatening situation in which a subject finds it effortful to remain 
calm (for example a dentist exam). There is a substantial body of evidence supporting the validity of the $0.1 \mathrm{~Hz}$ component for indicating mental effort, although the sensitivity of this method for changes in task load vs. changes in emotional load or stress is disputed by some authors (Nickel \& Nachreiner, 2003).

Mulder himself considered making this difference in this particular case as not practical, as an increased task load actually would correlate with an increase in emotional load. The relevance becomes obvious, however, when comparing the 0.1 $\mathrm{Hz}$ component method with subjective rating scales: as a part of the validation procedure for the RSME, Zijlstra (1993) compared the ratings on the RSME to the changes in the $0.1 \mathrm{~Hz}$ component in a laboratory setting and discovered a discrepancy of the two methods. While the conditions of a laboratory task were designed to induce different levels of task load, the resulting changes in mental workload were only picked up by the RSME. A more thorough analysis revealed that, apparently, the arousal of the subjects influenced the measured $0.1 \mathrm{HZ}$ component. When subjects were for example more aroused because they could easily succeed (win) at a game-like task, the $0.1 \mathrm{~Hz}$ component was actually reduced, which would originally be assumed to reflect a high level of experienced workload. Zijlstra came to the conclusion that, although both methods supposedly measured the same construct, the sensitivity of the RSME for differences in task load is much higher. In his interpretation, the $0.1 \mathrm{~Hz} \mathrm{HRV}$ component is rather an index of the transient feeling of strain and tension perceived during task performance, or of increased levels of arousal during task performance.

This discrepancy illustrates a conceptual problem with peripheral measurements of mental effort investment. While these measurements are easily accessible, they pose only indirect correlates of the actual variable that is supposed to be measured. Even if the $0.1 \mathrm{~Hz}$ component indeed accurately reflects arousal or strain that an operator perceives during task performance, this would make it an indicator of changes that compose only one part of the total experience of mental effort investment. This total experience is influenced by the investment of mental effort into all regulative processes that are necessary to perform a task. Peripheral variables might form an indicator of one or more aspects of mental effort investment, but they do not equal nor indicate the subjective experience of the operator. Hence, no single peripheral variable on itself would fully reflect the amount of subjectively experienced mental effort. Therefore, the role of such variables as a means to test validity of subjective rating scales by providing an account of subjectively experienced mental effort investment during task performance is at least severely limited.

\subsubsection{Metabolic indicators of mental effort investment}

Another approach to assess mental effort is to use metabolic measures which indicate the actual use of energy in form of glucose. Glucose is the bodies' main fuel. The human brain consumes a not at all marginal portion of this fuel during mental tasks. Fairclough and Houston (2004) investigated the possibility of using this fact to 
deduce the amount of exerted effort from the changes in blood glucose levels in their subjects during the execution of mental tasks. Their manipulation included differences in task load, but also prolonged task performance to induce time-ontask-effects (TOT), which change the total amount of mental work load by increasing the state load that the operator has to meet with his or her effort investment. The control measurement methods were the $0.1 \mathrm{~Hz}$ component described above as well participants' performance on the mental tasks. Fairclough and Houston utilized Stroop tasks with different levels of distraction to manipulate task load. As Fairclough and Houston state, blood glucose decreases as a function of task difficulty and time. Even though they did not observe a related decrease in performance over time, other studies have established the link between decreasing blood sugar and decreasing performance in high-demanding tasks of different sorts (Feldman \& Barshi, 2007).

With this approach, Fairclough and Houston tried to quantify the total invested mental effort by measuring the expenditure of the source of energy that fuels all mental processes on a neural level. This follows the assumption that the amount of invested effort is reflected in the amount of metabolized glucose. Additional evidence indicates that the decrease in performance shown by Fairclough and Houston (2004) is mainly due to a loss of self-regulative ability that does not depend on the precise nature of the task. In a series of experiments, Gailliot et al. (2007) showed that several kinds of self-regulative activities decrease blood glucose levels. The lower the levels of blood glucose, the lower an individual performed on a subsequent self-control task. This view of blood glucose as an indicator of energy consumption of the brain seems invitingly straightforward at first glance, as the role of glucose as an energy source for most processes in the human body is common knowledge. Yet, in case of the brain, energy supply and consumption is a quite complex process in which levels of blood glucose merely are one of several factors, which make the relation between blood glucose and neural functioning all but straightforward (Beedie \& Lane, 2011). For example, blood glucose is not directly available to the neurons, and both the extracellular fluid and the astrocytes in the human brain act as an intermediate energy storage, which introduces a delay for any influence of changing blood glucose levels (Gibson, 2007). This underlines a major limitation of peripheral variables as indicators of a psychological construct as mental effort: While those variables might ultimately be influenced by the metabolic processes underlying effort investment, the indirect nature of a variable`s relation to the psychological processes in question acts as what can best be described as a "filter" with unknown properties. Changes in the psychological process somehow result in changes in the peripheral variable; but without knowing the precise functional relation between the psychological process and the peripheral variable, it is not sound to treat changes in the level of the peripheral variable as unambiguous evidence that a change in for instance the level of mental effort investment has taken place. Levels of blood glucose, after all, are influenced as well by a multitude of other physiological processes. Hence, the validity of the approach to use blood 
glucose levels as indicator of previously invested effort on the one hand depends on the understanding of the precise mechanics that connect mental effort investment to changes in the level of blood glucose. Furthermore, the use of this approach is limited to settings in which the influence of other, secondary physiological processes on blood glucose can be controlled or ruled out.

Nevertheless, this approach poses an attempt to find not only an objective correlate of the subjective investment of mental effort, but to also find a correlate of the operator`s decreasing resources in relation to sustained performance using a peripheral indicator. What especially the results by Gailliot et al. (2007) show, above all, is that the ability to perform acts of effortful self-regulation is a general, meta-level ability of the human brain that is not specific to a certain task, and that depleting this resource results in performance decrease that is not specific to the domain of the task by which the resource was depleted in the first place. This is consistent with the notion that the concept of mental effort refers to a domainunspecific functional principle of brain functioning. At the same time, these results form a strong indicator for a biological basis of the regulative resource of mental effort. It seems thus warranted to take a closer look at the functioning of the human brain as the location in which the regulative action takes place. Peripheral indicators of energy consumption do indicate that, somewhere, a consumption of glucose has taken place. By using neuroimaging tools, we can investigate where exactly the neurons of the brain exhibit this glucose-consuming activity, and how this changes in the face of changing levels of mental effort investment. Knowing which neural structures are employed in the process of effort investment would help to bridge the gap between mental effort as a concept of brain functioning and actual, observable brain functioning.

\subsubsection{Neuroimaging: A more direct way of studying mental effort}

As became evident from the research into physiological correlates of mental effort investment, peripheral indicators suffer from the fact that they are only indirectly related to the process of interest. A more direct approach might therefore be beneficial. Mental effort investment happens in the brain, therefore measures of neural correlates of mental effort investment and its effects might be able to provide less perturbed indicators of the subjective experience.

Such investigations have a long tradition in psychological research: One of the earliest neuroscientific experiments, was conducted by Angelo Mosso in the late $19^{\text {th }}$ century, and was actually a basic study of the physiological correlates of mental effort: It measured the extra weight of blood in a person's head when the person engaged in effortful thinking. Mosso employed a simple device to measure the relative increase of blood volume in the brain: His setup consisted of a bench that was rested on a single pivot in the middle. The subject was placed carefully in a supine position on the bench, and the bench was balanced (see Fig. 5). Letting the subject perform a mental task would, according to Mosso, lead to increased blood flow to 
the brain, which would cause the head end of the bench to tip over. While the outcome of this particular experiment has been disputed, Mosso`s approach to measure a correlate of the activation of the whole brain is an example of an attempt to measure the combined effect of all factors that play a role in mental effort investment.

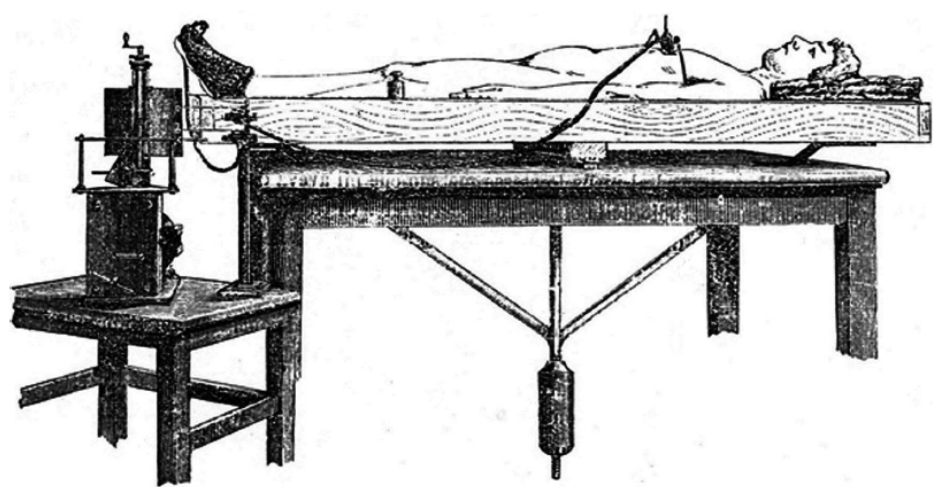

Fig. 5: Mosso's bench experiment. The participant was placed on a wooden bench that was pivoted in the middle, much like a see-saw. The bench was carefully balanced while the participant was at rest. Supposedly, cerebral activation would increase the total amount of blood in the brain, causing the bench to tip over at the head end.

Modern neuroscientific tools have advanced far beyond wooden bench setups, and they offer ways to investigate brain functioning in a more detailed way.

The most prominent for in-vivo research are Electroencephalography (EEG) and functional Magnetic Resonance Imaging (fMRI). In EEG, the electrical current associated with neuronal activity is measured by electrodes that are attached to the outside of the head. A commonly used technique in EEG research is the measurement of event-related potentials (ERP). ERP are essentially the electrical response that can be measured in relation to the start of an event, such as tone that is presented to the subject (Luck, Woodman, \& Vogel, 2000). FMRI measures local changes in oxygen saturation that have been found to correlate with the activation of local populations of neurons (Logothetis, Pauls, Augath, Trinath, \& Oeltermann, 2001). Those methods differ in their characteristics: EEG has a high temporal solution in the range of milliseconds, while its spatial resolution is coarse. The case of fMRI is the exact opposite, with a spatial resolution that can be in the submillimeter range, but a temporal resolution that is more in a range of about a second. With the help of these techniques, it is thus possible to assess changes in variables that are indicators of brain activity. This makes it possible to investigate the effects of mental effort investment directly in the brain during the acute experience of mental effort investment. Several studies have already been conducted to this end, and their results demonstrate that mental effort investment indeed affects a large number of parameters of brain functioning. 
A straightforward approach to study the effects of different levels of mental effort investment has been followed by a number of researchers. In this approach, the level of required mental workload is varied while some parameters of brain functioning are measured. Different levels of required mental workload can be achieved by several means. One possibility is to change the amount of effort that subjects invest into an unchanged task by altering the task instructions. An example of such an experiment can be found in a study by Mulert et al. (2008). In this study, subjects performed an auditory reaction task under with wither the instruction to perform the task in a relaxed manner or to react as fast and precise as possible, to induce low and high effort conditions, respectively. Simultaneously, brain activation was measured using both EEG and fMRI. The results showed an increase in the so-called N1 ERP component (a negative peak in the EEG signal roughly 100 milliseconds after an event). This increase in N1 amplitude was found to be related to increased activation in the Anterior Cingulate Cortex (ACC), as shown in the fMRI data. Another way to achieve different levels of mental workload is to manipulate a task so that it requires different levels of processing effort (think for example of changing the number of items a subject has to keep in working memory). Such manipulations have been used for example in working memory tasks (Owen, McMillan, Laird, \& Bullmore, 2005; Ragland et al., 2002), in visual discrimination tasks (Deary et al., 2004), but see also Duncan and Owen (2000) for an overview of the effects of changing levels of task difficulty on classical paradigms such as the Stroop task.

In summary, these studies have demonstrated that changing task demands entail effects on a wide range of brain areas, among which the anterior cingulate cortex (Mulert, Menzinger, Leicht, Pogarell, \& Hegerl, 2005) or the medial ventroand dorsolateral prefrontal cortices, the insula, and parts of the inferior parietal cortex (Ragland et al., 2002). It remains unclear, however, which role these specific neural structures play in the investment and experience of mental effort. Even stronger, the design of these studies does not allow to actually relate specific neural structures to mental effort, rather than to the specific cognitive processes that were performed by the participants of the respective studies. Is a specific structure more active in a condition with high workload because it is part of a neural mechanism that invests or monitors mental effort? Or is it simply more active because it processes information at a higher load? As all of the above studies only manipulate task load, only one of the two classes of loads that the total mental workload comprises is manipulated. The concept of mental effort, however, describes how the overall investment of a regulative resource is deployed. The crucial notion about this deployment is that any parameter in an operator can be the target of this regulation, not depending if this parameter is related to information processing or the regulation of mental state in order to perform a task. In order to identify neural structures which are responsible for the management of mental effort a different strategy needs to be employed, which includes both the manipulation of mental effort by varying both the task and the state load. 

Chapter 3

Making mental effort visible 
CHAPTER 3 


\subsection{The necessity to combine expertise from occupational psychology with neuroimaging methods}

The results of the studies described in the previous chapter show that a large number of brain areas can be shown to change their activation level when task demands are varied. This forms an obvious limitation to any approach that simply contrasts different levels in task demands: It can be difficult to separate activation that is specific for performing a task and activation that can be attributed to the experience and the investment of mental effort. Even when data from paradigms that use a number of different tasks are compared, the changes in brain functioning that are measured during task performance still reflect two things: the changes in brain function that occur due to performing the task, but also the changes in brain function that occur due to the act of investing mental effort. As a consequence, it is impossible to interpret these results in relation to the concept of mental effort, as changes in neural activity cannot be unambiguously attributed to changes in effort investment or the experience thereof. This limitation entails that the results harvested from experimental paradigms which only employ differences in task load only pose mostly isolated findings about the effect of a cognitive manipulation on a neural structure or a pattern of neural activation, without offering the possibility of understanding the more general process of effort investment and experience. Of course, most of the described studies were conducted with very specific research questions in mind, which in some cases explicitly focused on structure-function relationships, or even on the brain activation related to one single paradigm. It is only in the retrospective perspective of this discussion that their relevance to the study of mental effort becomes clearly visible. What this limitation demonstrates, however, is that in order to identify the neural correlates of mental effort, a careful design of paradigms utilizing the existing knowledge that the field of occupational psychology has amassed on mental effort is absolutely paramount. The concept of mental effort refers to two sorts of loads: The task load, which has been employed as an experimental variable in a number of neuroimaging studies, entailing aforementioned limitations, but also the state load. The state load describes the amount of effort investment an operator needs to make to regulate his or her state to be suitable for task performance. In the theoretical framework, this state load is pictured as depending on the amount of arousal (Mulder, 1986; see also figure 3 in chapter 1). A maladjusted level of arousal will need to be regulated and kept at a level suitable for performing a task, which poses additional workload, the so-called state load (see also section 1.3). Factors that influence the arousal level of a person can be manifold, such as intoxication, sleep length, food intake (think of the feeling of relaxation and reduced desire for activity that overcomes one after a huge meal). In the context of occupational research, one of the most prominent factors is the influence of sustained performance. Experimental evidence for the influence of prolonged performance can be found for example in a study by Zijlstra (1993) 
which was carried out to validate the RSME by demonstrating its sensitivity to both changes in task load and to changes in state load. In this study, Zijlstra used the RSME to measure the amount of effort that participants had to invest into a working memory task. Two groups of participants performed the same working memory task with an easy and a difficult condition. Afterwards the participants indicated the invested amount of effort on the RSME These two task conditions represented two different levels of task load. Additionally, however, the groups received different pre-treatments in order to manipulate their states: One group spend the several hours before the task with effortful activities, while the other group was ordered to abstain from any effortful activity. By this pre-treatment, Zijlstra (1993) sought to induce two different levels of state load, as the group of participants that performed effortful tasks before performing the working memory task would additionally have to regulate their arousal levels. The results of this study clearly show that not only the two task load conditions, but also the two state load conditions influence the amount of mental effort invested in the task, as indicated by the participants on the RSME. The RSME was thereby shown to measure effort investment in accordance with the concept of mental effort as modeled by Mulder (1986) in his integrated model of mental effort, as the RSME was able to pick up variations in the required amount of effort due to both changes in task and state load.

Thus, both in the theoretical accounts of mental effort and in the evidence provided by earlier research using self-report measurements of mental effort, state load is an integral, demonstrated factor influencing the total amount of mental effort an individual needs to invest to achieve successful task performance. Especially the paradigm implemented by Zijlstra (1993) provides a valuable example of how both the task load and the state load can be incorporated as controlled experimental variables in laboratory research of mental effort. It is exactly this expertise and experience from the field of occupational psychology which is needed to design an approach to extend our understanding of mental effort with neuroimaging techniques. Experimental paradigms which only employ an incomplete operationalization of the mental effort concept only yield very limited information about the neural processes related to mental effort. Using an experimental design in which both main factors that influence mental effort investment are incorporated in a controlled manner, however, offer the possibility to make use of the possibilities offered to us by neuroimaging methods.

This 'extension of the occupational psychologist's toolbox', in turn, is in our opinion a most necessary advance that needs to be taken in order to refine and extend the understanding of mental effort from the perspective of occupational psychology. Using the conventional methods of performance-based experimental paradigms and cognitive modeling, occupational psychology has arrived at what can be described as a peak. The modern conceptualizations of mental effort such as the integrated model of mental effort (Mulder, 1986) form an abstract, but apparently accurate description of the concept of mental effort. The central aspect of this model, the notion that a limited resource is fueling all regulation, remains an un- 
challenged central aspect of the concept of mental effort (Fairclough \& Mulder, 2011). Moreover, this conceptual notion has independently been incorporated in other areas of psychological research, corroborating the findings and conceptualizations in occupational psychology: The concept of a finite resource is also the central pillar of the strength model of self-control (Baumeister et al., 1994), in which it is termed the "limited resource assumption". It is the rich body of experimental literature related to the field of self-control and regulation which further demonstrates the need for employing additional research approaches, as the probability of substantially adding to our understanding of the nature of this limited resource by conducting additional research using conventional methods seems small.. The limited resource assumption has been continuously supported by the vast majority of the numerous studies that have been conducted to test it (Hagger et al., 2010). Hagger et al. (2010) analyzed 83 experimental studies, of which 81 showed results consistent with the assumption of a limited resource underlying regulative abilities. The majority of these studies followed a pattern of measuring performance on tow tasks, a primary task intended to deplete the subject's resources, and a secondary task on which, due to the previous depletion of resources, performance was shown to be decreased. A decrease in performance due to the depletion of resources becomes only apparent after the subjects are either not willing or not able to sustain the increased level of mental effort investment required to secure a stable level of performance (Meijman, 1997). Thus, it can be assumed that if we were to replicate these studies while measuring the amount of mental effort that the subjects need to invest, the results would yield similarly corroborative evidence supporting a model in which mental effort is a limited resource.

We have thus arrived at a point in the development of theoretical concept of mental effort as well as the related concept of self-regulation at which the resulting models have become convergent in their description of this aspect of the human information processing system. The experimental techniques and approaches that have delivered the evidence that lead to the formation of the converging models seem to be currently unable to provide any fundamental extension of the concepts of mental effort, or of self-regulation. At the same time, the relation of the concept of a limited resource with other, clearly influential, factors such as motivation or affect remains obscure. Alternative theories to the strength model have been proposing a more central role of affect and motivation in explaining the observed changes in self-regulative abilities in paradigms incorporating sustained performance (Hagger et al., 2010). Instead of focusing on a finite regulative resource, such alternative explanations assume that for example sustained performance builds up negative affect, which in turn prompts the individual to engage in behavior alleviating the negative mood (in the context of performance, the cessation of further increasing the negative affect by ceasing performance) (Bruyneel, Dewitte, Franses, \& Dekimpe, 2009), or that a subject's capacity is increasingly occupied to compensate a steady shift of the motivational balance in disfavor of sustained self-regulation (Meijman, 1997). These alternative explanations would technically also work under 
the assumption of a fixed capacity assumption in which the resource underlying self-regulation is not depleted, but rather progressively bound in compensating the increasing threat to performance. Yet, the experimental evidence still supports a finite resource model: lack of motivation or negative affect can unfold their detrimental effects on performance only when a person reaches a state of depletion in which the a person`s ability to engage in successful self-regulation is impaired (Bruyneel et al., 2009; Meijman \& Mulder, 1998). Hagger et al. (2010) name a number of additional factors such as fatigue or change in affect that are consistently observed to occur in when people performed sustained exhaustive tasks (Govorun \& Payne, 2006; Tice, Bratslavsky, \& Baumeister, 2001). These factors have been proposed as central elements of alternative theories that aim to explain the observed pattern of performance decrease. As one of the lead conclusions of their extensive review, they interpret the analyzed studies as supportive for the strength model. At the same time, the evidence is not sufficient to rule out alternative explanatory models. As Hagger et al. note, understanding the specific mechanisms that link sustained self-regulation to motivational or affective processes is paramount to understand the interplay of these different, clearly associated factors. Yet, as the authors point out, the lack of knowledge about the nature of the limited resource that underlies self-regulation forms a major hurdle for further research, as for example performance remains the main objective dependent variable that can be used to detect the depletion of the underlying resource.

In the context of mental effort, factors as fatigue, or changes in affect are included, as well as their influence on motivation, are an integral part of the concept. The basic assumption is that effort investment is based on the decision of the individual to adopt a goal and to willingly invest a certain amount of regulative resources into reaching the set goal. In this view, effortful self-regulation can even be used to counter factors such as mood, fatigue, and even provide a protection of the initial motivational state against the de-motivating effects of the other factors (Meijman \& Mulder, 1998). Models like the integrated model of mental effort (Mulder, 1986) fully accommodate the notion that any additional need for regulation in order to keep the individual in a state that allows performance requires additional effort in form of the state load. Together with the state load, the integrated model of mental effort thus provides a complete account of the changing amount of effort that an individual must invest to perform a sustained task. In this, the model pictures the relationship between task and operator as interactive, thus including the notion that the operator's state will change over time, and that, in turn, the operator might change his or her strategies or even alter the set task goals as well. What the integrated model of mental effort does not describe, however, is how exactly the concept of mental effort is linked to factors such as affect, motivation or fatigue. Akin to the research challenges identified for the strength model identified by Hagger et al. (2010), a truly integrated perspective on mental effort requires the understanding of how mental effort is linked to affective, cognitive and motivational processes. Research efforts into mental effort and into self-control seemingly 
have approached the same concept of a limited regulative resource: The concept of mental effort describes the mobilization of an energetic resource that, in the strength model of self-control, is applied to actively regulate one`s behavior. For the moment, the experimental evidence and theoretical considerations of both of these directions have arrived at a common challenge. This challenge is to understand exactly what limits the self-regulative power of the human brain. The crucial gap in our understanding is the lack of a physiological manifestation of this limited resource. Thereby, naturally, the possibilities to measure the investment of the limited resource are restricted. Performance can only indirectly hint at the depletion of the resource, while the relation of peripheral physiological indicators of its depletion such as blood glucose to the actual limited resource that underlies regulation is not sufficiently understood. The most direct estimation of the actual usage of mental effort that is available are self-reports that can be provided by operators. Yet, such self-reports cannot, on their own, teach us anything new about the abstract resource that underlies them.

What Hagger et al. (2010) identified as the course of action for overcoming the gap in knowledge for the strength model of self-control, "Identifying physiological analogs for ego depletion may offer a potential solution" (p. 205), is in our opinion the necessary next step in extending our understanding of mental effort. What we know is that the way that an individual is able to regulate him- or herself changes as an effect of sustained regulation. We thus assume that the brain of an individual functions differently before and after sustained investment of mental effort. In order to extend our knowledge about the nature of mental effort as a resource, we thus have to investigate how the use and consumption of this resource is reflected in the brain. The challenge is to isolate the changes in brain activation related to experienced investment of mental effort from the changes that are specific for the processes related to task performance. In order to achieve this goal, it is necessary to utilize both the possibilities offered to us by modern neuroimaging techniques, and the theoretically sound design of experimental manipulations of mental effort investment that have been successfully employed in cognitive investigations of the matter so far.

\subsection{A strategy to identify neural correlates of mental effort}

The identification of neural correlates of mental effort as such is a quite complex problem. The only quantification available is the subjective, self-reported experience of operators, which additionally is given only after performance on a laboratory task has actually taken place. Thus, normally, we would only be able to obtain a quantification of mental effort after the act of investing effort has taken place, and we would have to rely on the assumption that the post-hoc evaluation reflects the acute experience of the operator while performing the task. However, the possibil- 
ity to measure brain activation without interfering with a subject's task performance offers us a way to evaluate the similarity of experience and post-hoc selfevaluation of mental effort investment. Brain activation can be measured during task performance itself, but also during the process of rating one`s own mental effort investment. Thereby, it can be assessed if experiencing mental effort investment acutely and the act of evaluating it afterwards share neural structures.

The existence of such a specialized, shared mechanism for experience and selfevaluation of mental effort has been proposed earlier by Naccache et al (2005) based on observations in a patient. This patient, abbreviated RMB, was found to lack any sense of mental effort investment, following an ischemic lesion. Her cognitive abilities were largely preserved, and she was able to react to changes in task demands in a flexible way. Also, RMB was able to deduce changes in task demands on a pure cognitive level. However, she reported a distinct lack of experiencing the investment of mental effort, or any sense of difference across task conditions that obviously were designed to create different levels of task load. Presented with different task conditions that only covertly varied in their associated task load, thereby precluding a cognitive deduction of the different task levels, patient RMB was not able to give any account of differences in the required effort investment at all. Based on this evidence, Naccache et al. (2005) propose the existence of a specialized, dissociable mechanism that underlies both the experience and the selfevaluation of mental effort. While the extensive nature of RMB`s lesion does not allow to evaluate in how far identical neural structures would be responsible for both the deficit in experience and in post-hoc evaluation, her case provides strong evidence for specialized common neural correlates of mental effort investment and evaluation.

The dissociation displayed by RMB allowed for the opportunity to isolate neural correlates of mental effort evaluation from other evaluative processes related to a previously performed task. An experimental paradigm that confronts a participant with different levels of task load would induce different levels of mental effort investment, which the participant later had to indicate using a commonly used selfreport instrument such as the RSME. By measuring the brain activation during this rating process and contrasting it with brain activation when a participant rates task difficulty, we would reproduce the functional difference that manifested in the dissociation of patient RMB. We would then be able to subsequently study the behavior of the thereby identified neural structures under varying levels of acute mental effort investment during performance. This necessitates the careful construction of a paradigm based on the fact that mental effort is the communal resource for meeting all regulative need. Paradigms that exclusively manipulate task load will find widespread activation over a large number of brain areas (Duncan \& Owen, 2000). However, differences in task load are only one class of need for regulative action that an individual faces. The solution is to design a paradigm that allows for a manipulation in both task and state load. Thereby, an additional criterion, namely the sensitivity to changes in state load could offer a way to identify changes in brain 
activation that reflect changes in subjectively experienced effort during task performance. This would allow testing the assumption that post-hoc evaluations of mental effort investment and acute experience thereof indeed share common neural structures. More important, the combination of the subjective self-report technique and the measured brain activation would allow, for the first time, to actually relate the experience of effort investment to localized neural changes. Taking into account findings from other subjective modalities, it is reasonable to hypothesize that parametrical changes in the subjective experience of mental effort are reflected in parametrical changes in brain activation. Similar observations have been made in several subjective modalities such as emotional valence (Anders, Lotze, Erb, Grodd, \& Birbaumer, 2004) or pain perception (Kong et al., 2006), where the strength of the subjective experience was reflected in changes in the activation of related brain areas.

Most important, measuring brain activation in relation to sustained performance might also offer a way to better understand the effects that investing mental effort has on the way that the human brain functions. Sustained investment of mental effort, no matter in which respect, on which task, or in which context, has been shown to change the way that people are able to bring their actions into accordance with their goals. A fundamental change in the ability of healthy people to regulate their own behavior takes place, accompanied by measurable changes in the psychological constructs which surround self-regulation (Hagger et al., 2010). Achieving a systemic, truly integrated perspective on mental effort is only possible by understanding how the functioning of the brain is changed by sustained effort investment. Individually, neural correlates of affective or motivational processes have been identified (Anders et al., 2004; Taylor et al., 2004). Yet, all of these individual processes are embedded in the "computational platform" of the brain. More recent techniques in functional neuroimaging have made it possible to examine not only contrasting differences in brain activation, but also the way that brain regions function together (McKeown et al., 1998; Esposito et al., 2008). Information processing in the brain, put brief, relies on the fact that different brain regions can organize themselves in a flexible manner into functional networks. A large number of neurological conditions typically associated with fatigue, like for example multiple sclerosis, have been associated with changes in functional connectivity in the functional brain networks (van den Heuvel \& Hulshoff Pol, 2010; Stam \& van Straaten, 2012). Also in healthy participants, classical debilitating factors as intoxication by alcohol have been demonstrated to affect the functional connectivity in the brain in a dosedependent fashion (Esposito et al., 2010), and intoxication-mediated attenuation in the reactivity of brain areas that form a part of such functional networks are associated with decreases in cognitive performance in intoxicated participants (Marinkovic, Rickenbacher, Azma, \& Artsy, 2012). We have thus reason to hypothesize that the changes in self-regulative capabilities and fatigue associated with sustained investment of mental effort will be reflected in measurable changes in functional networks in the brain. Investigating this so-called functional connectivity in 
relation to sustained effort investment will likely show how the brain is affected by a sustained, depleting investment of effort.

Summarizing, it is thus possible to test the hypotheses stating that

- There are neural correlates of subjectively experienced mental effort investment that are sensitive to changes in task and state load in the same way as subjective rating scales, in this case the RSME.

- Experiencing and evaluating mental effort investment recruits shared neural structures.

- The effects of sustained task performance on brain functioning can be identified using neuroimaging methods.

Over the course of the next three chapters of this book, I present three studies in which these hypotheses are tested. The studies that we conducted were designed while building on all the efforts that have been made by other researchers before. Testing the hypotheses that became immanent from this earlier work will be, most important, a further step in extending our understanding of mental effort, and of the ways that we measure it. Measurement devices that are used to assess mental effort investment, in particular the RSME, provide valuable data about the subjective experience of people in the process of investing mental effort. In turn, by combining such proven instruments with more recent neuroimaging techniques, we also have the opportunity to learn more about instruments as the RSME as well. Yet, the implications of extending our fundamental understanding of the concept of mental effort stretch much further than the mere direct theoretical considerations. As occupational psychologists, one of our central goals is to understand and improve the relation of individuals with the tasks that they perform. Gaining a more holistic understanding of how the act of performing a task - any task - changes the individual in such a fundamental respect as to alter the functioning of the brain is simply imperative to our continued effort to pursue this goal. 


\section{References}

Anders, S., Lotze, M., Erb, M., Grodd, W., \& Birbaumer, N. (2004). Brain activity underlying emotional valence and arousal: A response-related fMRI study. Human Brain Mapping, 23(4), 200-209. doi:10.1002/hbm.20048

Baumeister, R. F., Bratslavsky, E., Muraven, M., \& Tice, D. M. (1998). Ego depletion: Is the active self a limited resource? Journal of personality and social psychology, 74, 1252-1265.

Baumeister, R. F., Heatherton, T. F., \& Tice, D. M. (1994). Losing control. Academic Press San Diego, CA.

Baumeister, R. F., Vohs, K. D., \& Tice, D. M. (2007). The Strength Model of Self-Control. Current Directions in Psychological Science, 16(6), 351-355. doi:10.1111/j.1467-8721.2007.00534.x

Beedie, C. J., \& Lane, A. M. (2011). The Role of Glucose in Self-Control: Another Look at the Evidence and an Alternative Conceptualization. Personality and Social Psychology Review, 16(2), 143-153. doi: $10.1177 / 1088868311419817$

Borg, G. (1972). A ratio scaling method for interindividual comparisons. University of Stockholm, Institute of Applied Psychology.

Botvinick, M. M., \& Rosen, Z. B. (2008). Anticipation of cognitive demand during decision-making. Psychological Research Psychologische Forschung, 73(6), 835-842. doi:10.1007/s00426-008-0197-8

Brookhuis, K. A., \& de Waard, D. (2010). Monitoring drivers' mental workload in driving simulators using physiological measures. Accident Analysis \& Prevention, 42(3), 898-903.

Bruyneel, S. D., Dewitte, S., Franses, P. H., \& Dekimpe, M. G. (2009). I felt low and my purse feels light: depleting mood regulation attempts affect risk decision making. Journal of Behavioral Decision Making, 22(2), 153-170. doi:10.1002/bdm.619

Cooper, G. E., \& Harper Jr, R. P. (1969). The use of pilot rating in the evaluation of aircraft handling qualities. DTIC Document.

Damasio, A. (1999). The feeling of what happens. New York: Harcourt Brace \& Co.

Deary, I. J., Simonotto, E., Meyer, M., Marshall, A., Marshall, I., Goddard, N., \& Wardlaw, J. M. (2004). The functional anatomy of inspection time: an event-related fMRI study. Neuroimage, 22(4), 1466-1479.

Duncan, J., \& Owen, A. M. (2000). Common regions of the human frontal lobe recruited by diverse cognitive demands. Trends in neurosciences.

Esposito, F., Aragri, A., Pesaresi, I., Cirillo, S., Tedeschi, G., Marciano, E., ... Di Salle, F. (2008). Independent component model of the default-mode brain function: combining individual-level and populationlevel analyses in resting-state fMRI. Magnetic Resonance Imaging, 26(7), 905-913. doi:10.1016/j.mri.2008.01.045

Esposito, F., Pignataro, G., Di Renzo, G., Spinali, A., Paccone, A., Tedeschi, G., \& Annunziato, L. (2010). Alcohol increases spontaneous BOLD signal fluctuations in the visual network. NeuroImage, 53(2), 534-543. doi:10.1016/j.neuroimage.2010.06.061

Fairclough, S. H., \& Houston, K. (2004). A metabolic measure of mental effort. Biological Psychology, 66(2), 177-190.

Fairclough, S. H., \& Mulder, L. M. J. (2011). Psychophysiological Processes of Mental Effort Investment. In R. Wright \& G. Gendolla (Eds.), How Motivation Affects the Cardiovascular Response: mechanisms and applications. Washington DC: American Psychological Association.

Feldman, J., \& Barshi, I. (2007). The Effects of Blood Glucose Levels on Cognitive Performance: a Review of Literature. Moffett Field, California, NASA Ames Research Center.

Gailliot, M. T., Baumeister, R. F., DeWall, C. N., Maner, J. K., Plant, E. A., Tice, D. M., ... Schmeichel, B. J. (2007). Self-control relies on glucose as a limited energy source: Willpower is more than a metaphor. Journal of personality and social psychology, 92(2), 325.

Gibson, E. L. (2007). Carbohydrates and mental function: feeding or impeding the brain? Nutrition Bulletin, 32(s1), 71-83.

Govorun, O., \& Payne, B. K. (2006). Ego-Depletion and Prejudice: Separating Automatic and Controlled Components. Social Cognition, 24(2), 111-136.

Hagger, M. S., Wood, C., Stiff, C., \& Chatzisarantis, N. L. D. (2010). Ego depletion and the strength model of self-control: A meta-analysis. Psychological Bulletin, 136(4), 495-525. doi:10.1037/a0019486

Hart, S. G., \& Staveland, L. E. (1988). Development of NASA-TLX (Task Load Index): Results of empirical and theoretical research. Human mental workload, 1, 139-183. 
Hockey, G. R. J. (1997). Compensatory control in the regulation of human performance under stress and high workload: A cognitive-energetical framework. Biological Psychology, 45(1-3), 73-93. doi:doi: 10.1016/S0301-0511(96)05223-4

Kahneman, D. (1973). Attention and effort. Englewood Cliffs, NJ: Prentice-Hall.

Kong, J., White, N. S., Kwong, K. K., Vangel, M. G., Rosman, I. S., Gracely, R. H., \& Gollub, R. L. (2006). Using fMRI to dissociate sensory encoding from cognitive evaluation of heat pain intensity. Human Brain Mapping, 27(9), 715-721. doi:10.1002/hbm.20213

Logothetis, N. K., Pauls, J., Augath, M., Trinath, T., \& Oeltermann, A. (2001). Neurophysiological investigation of the basis of the fMRI signal. Nature, 412(6843), 150-157.

Luck, S. J., Woodman, G. F., \& Vogel, E. K. (2000). Event-related potential studies of attention. Trends in Cognitive Sciences, 4(11), 432-440. doi:10.1016/S1364-6613(00)01545-X

Marinkovic, K., Rickenbacher, E., Azma, S., \& Artsy, E. (2012). Acute alcohol intoxication impairs topdown regulation of stroop incongruity as revealed by blood oxygen level-dependent functional magnetic resonance imaging. Human Brain Mapping, 33(2), 319-333. doi:10.1002/hbm.21213

McKeown, M. J., Makeig, S., Brown, G. G., Jung, T. P., Kindermann, S. S., Bell, A. J., \& Sejnowski, T. J. (1998). Analysis of fMRI data by blind separation into independent spatial components. Human brain mapping, 6(3), 160-188.

Meijman, T. F. (1989). Neuroendocrine activatie: Mobilisatie en compensatie. In T. F. Meijman \& J. Furda (Eds.), Mentale belasting en werkstress: een arbeidspsychologische benadering. Van Gorcum.

Meijman, T. F. (1997). Mental fatigue and the efficiency of information processing in relation to work times. International Journal of Industrial Ergonomics, 20(1), 31-38.

Meijman, T. F., \& Mulder, G. (1998). Psychological aspects of workload. Handbook of work and organizational psychology: Work psychology, 2, 5-33.

Moray, N. (1967). Where is capacity limited? A survey and a model. Acta Psychologica, 27, 84-92.

Mulder, G. (1980). The heart of mental effort. Unpublished doctoral dissertation, University of Groningen, Groningen, The Netherlands.

Mulder, G. (1986). The concept and measurement of mental effort. In G. R. J. Hockey, A. W. K. Gaillard, \& M. G. H. Coles (Eds.), Energetics and Human Information Processing. Dordrecht: Martinus Nijhoff.

Mulert, C., Menzinger, E., Leicht, G., Pogarell, O., \& Hegerl, U. (2005). Evidence for a close relationship between conscious effort and anterior cingulate cortex activity. International Journal of Psychophysiology, 56(1), 65-80.

Mulert, C., Seifert, C., Leicht, G., Kirsch, V., Ertl, M., Karch, S., ... others. (2008). Single-trial coupling of EEG and fMRI reveals the involvement of early anterior cingulate cortex activation in effortful decision making. Neuroimage, 42(1), 158-168.

Naccache, L., Dehaene, S., Cohen, L., Habert, M. O., Guichart-Gomez, E., Galanaud, D., \& Willer, J. C. (2005). Effortless control: executive attention and conscious feeling of mental effort are dissociable. Neuropsychologia, 43(9), 1318-1328.

Nickel, P., \& Nachreiner, F. (2003). Sensitivity and Diagnosticity of the 0.1-Hz Component of Heart Rate Variability as an Indicator of Mental Workload. Human Factors: The Journal of the Human Factors and Ergonomics Society, 45(4), 575-590. doi:10.1518/hfes.45.4.575.27094

O'donnell, R., \& Eggemeier, F. T. (1986). Workload assessment methodology. Measurement Technique, 42, 5.

Owen, A. M., McMillan, K. M., Laird, A. R., \& Bullmore, E. (2005). N-back working memory paradigm: A meta-analysis of normative functional neuroimaging studies. Human Brain Mapping, 25(1), 46-59. doi:10.1002/hbm.20131

Ragland, J. D., Turetsky, B. I., Gur, R. C., Gunning-Dixon, F., Turner, T., Schroeder, L., ... Gur, R. E. (2002). Working memory for complex figures: An fMRI comparison of letter and fractal n-back tasks. Neuropsychology, 16(3), 370.

Reid, G. B., Eggemeier, F. T., \& Shingledecker, C. A. (1982). Subjective workload assessment technique. DTIC Document.

Rook, J. W., \& Zijlstra, F. R. H. (2006). The contribution of various types of activities to recovery. European Journal of Work and Organizational Psychology, 15(2), 218-240. doi:10.1080/13594320500513962 
Sanders, A. (1983). Towards a model of stress and human performance. Acta psychologica, 53(1), 61-97.

Sartori, G., \& Umiltà, C. (2000). How to Avoid the Fallacies of Cognitive Subtraction in Brain Imaging. Brain and Language, 74(2), 191-212. doi:10.1006/brln.2000.2334

Shulman, G. L., Corbetta, M., Buckner, R. L., Raichle, M. E., Fiez, J. A., Miezin, F. M., \& Petersen, S. E. (1997). Top-down modulation of early sensory cortex. Cerebral Cortex, 7(3), 193-206. doi:10.1093/cercor/7.3.193

Staal, M. A. (2004). Stress, cognition, and human performance: A literature review and conceptual framework. Ames Research Center.

Stam, C. J., \& van Straaten, E. C. W. (2012). The organization of physiological brain networks. Clinical Neurophysiology, 123(6), 1067-1087. doi:10.1016/j.clinph.2012.01.011

Taylor, S. F., Welsh, R. C., Wager, T. D., Luan Phan, K., Fitzgerald, K. D., \& Gehring, W. J. (2004). A functional neuroimaging study of motivation and executive function. Neurolmage, 21(3), 1045-1054. doi:10.1016/j.neuroimage.2003.10.032

Tice, D. M., Bratslavsky, E., \& Baumeister, R. F. (2001). Emotional distress regulation takes precedence over impulse control: If you feel bad, do it! Journal of Personality and Social Psychology, 80(1), 53-67. doi:10.1037//0022-3514.80.1.53

Van den Heuvel, M. P., \& Hulshoff Pol, H. E. (2010). Exploring the brain network: A review on restingstate fMRI functional connectivity. European Neuropsychopharmacology, 20(8), 519-534. doi:10.1016/j.euroneuro.2010.03.008

Veltman, J. A., \& Gaillard, A. W. K. (1996). Physiological indices of workload in a simulated flight task. Biological psychology, 42(3), 323-342.

Verwey, W. B., \& Veltman, H. A. (1996). Detecting short periods of elevated workload: A comparison of nine workload assessment techniques. Journal of Experimental Psychology: Applied, 2(3), 270-285. doi:doi:10.1037/1076-898X.2.3.270

Winwood, P. C., Bakker, A. B., \& Winefield, A. H. (2007). An Investigation of the Role of Non-Work-Time Behavior in Buffering the Effects of Work Strain. Journal of Occupational and Environmental Medicine, 49(8), 862-871. doi:10.1097/JOM.0b013e318124a8dc

Zijlstra, F. R. H. (1993). Efficiency in Work Behavior: A Design Approach for Modern Tools. Delft: Delft University Press.

Zijlstra, F. R. H., \& Sonnentag, S. (2006). After work is done: Psychological perspectives on recovery from work. European Journal of Work and Organizational Psychology, 15(2), 129-138. doi:10.1080/135943205005138 



\section{Chapter 4}

\section{Neural correlates of mental effort evaluation - Involvement of structures related to self-awareness}

Published as Otto, T., Zijlstra, F. R. H., \& Goebel, R. (2012). Neural correlates of mental effort evaluation - Involvement of structures related to self awareness. Social Cognitive and Affective Neuroscience. doi:10.1093/scan/nss136 


\begin{abstract}
Mental effort is a limited resource which must be invested in order to perform mental tasks. The amount of mental effort investment that an individual experiences during task performance can be measured afterwards with the help of self-rating scales.

Earlier research suggests that integration of information about somatic state changes is crucial for the self-evaluation of mental effort investment. Damage to the pathways which convey information about somatic state changes can lead to an inability to self-evaluate mental effort investment, while conceptually similar evaluations of task difficulty can still be performed.

We used fMRI to investigate brain activation while subjects rated their mental effort investment and the difficulty of a previously performed task. Our results show stronger activation of the left anterior insular cortex (aIC) during evaluation of mental effort. Additionally, the activity in left aIC during task performance was modulated by changes in task demand in a similar way as the self-ratings of mental effort. We argue that aIC does not only play a role in the integration of self-related information during self-evaluation of mental effort investment, but that left aIC might also be involved in the experience of mental effort during task performance.
\end{abstract}




\subsection{Introduction}

\subsubsection{Scope of the study}

Mental effort is generally experienced while performing any sort of mental task, from compiling a grocery list to planning a scientific study. It is described as spending energy and consequently experiencing a feeling of strain, thus ultimately investing a limited energetic resource to perform a mental task (Zijlstra, 1993). Damasio (1999) considered mental effort to be not an emotion as such, but rather a feeling which refers to a conscious appraisal of one's own state. The amount of mental effort experienced while engaging in a task is determined by a variety of factors not limited to its mere computational demands (Mulder, 1986). Being aware of levels of mental effort investment provides information about the sustainability of a current activity. This awareness also makes it possible to evaluate the level of mental effort investment related to a task, and to communicate it.

The goal of this article is to identify neural structures that are involved in the process of mental effort evaluation. We will briefly describe the concept and measurement of mental effort. We will identify two crucial functions of a neural mechanism that enables mental effort evaluation. Evidence from research into brain areas with matching functional characteristics are then used to form hypotheses about the role of these areas in mental effort evaluation that will be tested using functional magnetic resonance imaging (fMRI).

\subsubsection{The concept of mental effort and its implications for neural structures}

Engaging in any mental task confronts an individual with a variety of demands. The integrated model of mental effort (Mulder, 1986) divides these in two classes of demands: The task load, which is related to the control of mental processes in order to produce the desired result, and the state load, which refers to all compensatory control that needs to be invested to reach and maintain a suitable working state. The actively invested control that an individual deploys to meet the combination of these two loads is referred to as mental effort. Investment of mental effort is accompanied by a subjective feeling of strain, and sustained high mental effort investment results in negative affect and fatigue (Hockey, 1997). The self-evaluation of mental effort investment has been described as a quasi-emotional appreciation of one's own state, reflecting the changes that occur in the state due to meeting the mental workload (Damasio, 1999).

The ability to self-evaluate one's own mental effort investment is necessary to manage one's performance in a sustainable way regarding one's own state. The existence of such an ability to intuitively self-assess the amount of mental effort related to the execution of a task has been demonstrated, and it is used routinely in occupational psychology. Modern tools measuring workload such as the rating 
scale mental effort (RSME; (Zijlstra, 1993) make use of this feature to provide an assessment of regulative effort in real-life work environments. Yet, to identify the impact of each of the many influences a subject faces in a work environment, it is necessary to understand the mechanism that leads to the coherent subjective experience of mental effort. Identifying the brain areas which are involved in the evaluation process provides an overview of the components of this mechanism.

By outlining necessary capacities of such an evaluative mechanism, it is possible to search for brain areas which have been shown to play a role in similar functions. Two crucial capacities of a mechanism for evaluating mental effort can be derived from the theoretical model of mental effort and from research into its subjective measurement. On the one hand, the mechanism must have the capacity to monitor different information streams that carry information about the state of the individual and to integrate this information into a coherent impression. Stressors related to task execution can affect all domains in the experience of a subject, from bodily homeostasis to cognitive resources. Thus, the integration of information originating from different domains is necessary to give an indication of mental effort. A brain region involved in joining these different information streams will need input from the respective regions that are concerned with the lower processing stages of this information.

The second necessary capacity of the mechanism is to make the result of the integrative process available for self-evaluation. It enables the subject to become aware of recently experienced mental effort levels and to communicate it. Conceptionally, self-evaluation of mental effort investment is a recall of the effect of the act of performing a task on the state of the self. The state of the self is thus used as a reference point, and changes in this state due to task performance are attributed to the investment of mental effort. Scales such as the RSME rely on the fact that this self-evaluation of mental effort can be performed fast and intuitively.

\subsubsection{A possible role for structures related to self-awareness}

Evidence from earlier research (see Craig (2009) for a review) suggests the insular cortex (IC), especially the anterior part, as a likely candidate region to provide the described necessary functionality. The IC receives input from a vast number of cortical and subcortical regions that provide it with information about the somatic, emotional and cognitive aspects of the state of the individual. The IC integrates this information in a posterior to anterior order. The anterior IC (aIC) thus receives and integrates information about all major aspects of an individual's state, which would enable it to determine the changes in this state due to mental effort investment. Thereby, it would fulfill the first criterion for a mental effort evaluation mechanism.

The second criterion, namely the capacity of a mechanism to perform a selfreferential evaluation, initially makes the involvement of an additional number of regions seem plausible. In particular, a set of regions termed Cortical Midline Structures (CMS; (Northoff \& Bermpohl, 2004), consisting of the anterior and posterior 
cingulate cortices/precuneus and the medial prefrontal cortex have been found to be activated in paradigms contrasting self-and other related information (see (van der Meer, Costafreda, Aleman, \& David, 2010) or Lieberman (2010) for a review).

While these regions react to changes in the degree of general self-reference, studies specifically investigating emotional self-awareness and interoception rather point towards a prominent role of aIC in this specific sub-domain of self-awareness.

Craig (2009) compared the results of several studies which employed experimental paradigms centered around awareness of self-state or emotions. In his review, Craig proposed that due to the extended integrative nature of aIC, it possesses the ability to essentially create a comprehensive momentary meta-representation of the subjective state of the self. Of particular interest in this context is the notion that overlapping regions in aIC have been shown to be reactive to changes in emotion as well as to interoceptive awareness (Zaki, Davis, \& Ochsner, 2012). A strong relation of somatic information and the subjective self-appraisal of one's state has been proposed earlier (Damasio, 1999). The results of Zaki et al. (2012) demonstrate that experience of emotion and attending to one's heartbeat actually do activate overlapping areas in aIC in the same participants, while a number of earlier studies have shown a connection between interoceptive awareness and emotional reactivity (see Herbert and Pollatos (2012) for a review).

For one emotion, namely anxiety, a mechanism has been proposed in which aIC compares states of the bodily self at different times, signaling the occurrence of unexpected discrepancies between predicted and actual state (Paulus \& Stein, 2006). Further evidence of such a functionality of aIC comes from its consistent, overlapping activation during both actual experience of pain and empathizing with other's pain, the latter of which involves emulating the state changes caused by the pain stimulus (Singer, Critchley, \& Preuschoff, 2009). Such a state-comparing functionality of aIC would fit very closely with the conceptual description of selfevaluation of mental effort investment, especially as mental effort investment has long been known to cause changes in the somatic state of an individual (Fairclough \& Mulder, 2011).

The involvement of several parts of the IC in evaluative processes has already been demonstrated in a number of paradigms. In a meta-analysis of studies investigating brain activation during explicit emotional evaluation, (Lee \& Siegle, 2009) identified the IC as being activated specifically during evaluation of one's own emotion-related states by subjects. Although the locations of activated clusters in IC vary over different paradigms used in the analyzed studies, the computed peak activation is located towards aIC.

These two features, the integration of information from different domains and the proposed relation to emotional awareness and self-evaluation, provide the aIC with two prerequisites for mental effort evaluation according to the outlined theory above: First, necessary information about the state of the self, most important the bodily aspect, are projected to it. Second, the combined evidence suggests a func- 
tionality of aIC in which different states are compared, thereby enabling it of assessing the influence of task performance on the state of the self.

Findings from a patient case study (Naccache et al., 2005) provide further evidence supporting the crucial role of bodily state information in the self-evaluation of mental effort. In this case, it was demonstrated that mental effort evaluation is dissociable from other evaluations concerning a recently performed task. After suffering an extensive lesion in the left anterior cingulate cortex (ACC), patient RMB was left with a specific impairment concerning her ability to evaluate her own invested mental effort. While she had no problems reasoning about the various difficulty levels of a task in terms of its computational demands, she did report to not feel any changes in her sense of mental effort investment. According to the interpretation of the authors, the function of the ACC is mainly the generation of emotional somatic markers in coordination with left ventromedial prefrontal regions; due to the lesion, these structures were disconnected which lead to the described inability to perform self-evaluation of mental effort investment. While in this case a lesion in ACC was responsible for the functional deficit, results from several studies reviewed by Craig (2009) showed no significant changes in ACC activation in relation to paradigms stimulating awareness of the self. Thus, in our view, ACC performs a necessary prerequisite role in self-related evaluation processes, but the main role of integrating and evaluating bodily information is performed by aIC.

Nevertheless, this case study presents a valuable observation that can be used to study the neural correlates of mental effort self-evaluation. The functional dissociation of evaluating task difficulty and invested effort offer a narrow contrast that can be used to assess the role of aIC in mental effort evaluation. While the evaluation of task difficulty seems similar to evaluating the mental effort, there is a crucial difference: Difficulty concerns the objective demands of a task, as perceived and estimated by the subject in a purely rational manner. Mental effort evaluation concerns the same task-sided loads, but it adds the subject itself in the evaluation. The subjective appraisal of one's own state is a necessary element in this process (Zijlstra, 1993). Therefore, the two key aspects of mental effort evaluation, namely integration of information across somatic/emotional/cognitive domains and the involvement of emotional self-awareness, do not play a prominent role in the evaluation of task difficulty. Thus, structures that support these two aspects of mental effort evaluation should be more active during the evaluation of mental effort than during task difficulty evaluation.

Still, both evaluations aim at the same recent task performance, and it has been demonstrated that nearly identical Visual Analogue Scale (VAS) interfaces can be used to assess the magnitudes of both effort and difficulty (Yeo \& Neal, 2004). Among the available tools for measuring mental effort, a one-dimensional VAS has the advantage of minimal intrusion, while, as proven for the RSME, still providing high sensitivity (Verwey \& Veltman, 1996). 


\subsubsection{Goal of the study and hypotheses}

The goal of our study was to provide insight in the neural structures that are activated stronger during the evaluation of mental effort than during the evaluation of task difficulty. We devised a design that makes use of the contrast between difficulty and mental effort evaluation. The paradigm employed a common working memory task, the n-back task (Gevins \& Cutillo, 1993). This task was used on three different levels of difficulty. Earlier findings demonstrated that ratings of both difficulty and amount of mental effort would be highly correlated (Yeo \& Neal, 2004) and would correspond to the memory load of the n-back task in a parametric fashion.

\section{Hypothesis 1:}

The difficulty and mental effort rating scores will show a significant correlation and will follow the parametric changes in task load.

The evaluation of a recent task using a VAS is in itself a complex process. The experience must be recalled, it must be evaluated, the outcome must be translated to a value on a scale, and finally a cursor must be moved to the chosen location on the scale. Lee and Siegle (2009) provided an overview of regions which, independent of the actual emotional content, would support the mere performance of a rating task as such. This includes expected task interface specific networks, which in our study would refer to networks engaged in visuo-motor coordination. The rating process itself relies on the recall of information over the task and on attention-related and higher cognitive functions to reflect and evaluate this information.

\section{Hypothesis 2:}

During both rating tasks, areas associated with visual and motor activity, attention, working memory and higher cognitive functioning will be activated. Specifically, this should involve areas in the anterior and posterior cingulate cortex (ACC/PCC) and the dorsolateral prefrontal cortex (dlPFC).

The main focus of our study concerned the contrast between those two rating conditions. Both the theoretical accounts of mental effort (Mulder, 1986) and the findings in patient RMB of Naccache et al. (2005) make it plausible that integration of information is a crucial part of mental effort evaluation. We propose that the aIC is suited to fulfill this role. The role of the aIC in self-awareness (Craig, 2009) makes it furthermore plausible that it serves to make the outcome of this process accessible to the subject as a part of the awareness of one's own state.

We, thus, expected this region to show stronger activation during mental effort evaluation than task difficulty evaluation. 
Hypothesis 3:

The aIC will be activated more during the evaluation of mental effort than during evaluation of task difficulty.

\subsection{Methods}

\subsubsection{Subjects}

14 Dutch - speaking subjects (3 male, 11 female, mean age 21.8 years, 12 right handed and 2 left-handed as measured by Edinburgh handedness inventory) were recruited using standard criteria for MRI safety and suitability for the experiment (no reports of mental health or extensive vision or motor problems). In order to minimize the chance for artifacts in the lower regions of the frontal cortex, we also excluded candidates with orthodontic retainers. Screened subjects were invited for the testing session. All subjects gave informed consent before the start of the experiment and were rewarded with either a fixed monetary reward or with a fixed number of student participation points.

2 male, right-handed subjects were excluded from analysis because of movement artifacts in the functional scans that exceeded the limits described under 4.2.3.1. This cut back our total sample size to 12 , yet this reduction is far less severe than the effects of including low-quality data sets.

\subsubsection{Task and procedure}

At the beginning of the experiment, the task was explained and practiced in a short version (one block of each condition) under supervision of the experimenter to check proper understanding. Subsequently, subjects were placed in the MRI scanner and performed 2 sessions of 15 task blocks each. The duration of one session was approximately 28 minutes. Between the two sessions, a T1 - weighted anatomical scan of the brain was carried out while the subjects were allowed to rest. The employed task was a version of the n-back task. Subjects saw letters appearing one after another on a computer screen. Subjects had to indicate if the letter currently presented on screen was identical to a letter that was presented either one, two or three letters back. These conditions were referred to as "1-back", "2-back" and "3back" conditions, respectively. N-back paradigms have been used to induce different levels of load in a number of previous studies (Owen, McMillan, Laird, \& Bullmore, 2005). The conditions were quasi-randomized, with 5 instances of each condition in each of the two sessions of the experiment.

At the beginning of each block, an instruction screen told subjects which condition they would encounter. Subjects than had the task of responding to 20 letters with either left or right button press to indicate that the letters were identical or 
not. After each letter they received feedback by either being presented with a green "Right" or a red "Wrong" for $500 \mathrm{~ms}$. The letters in question appeared on screen for $2000 \mathrm{~ms}$, which was also the response window. After each block of 20 trials, the subjects were given two consecutive VAS to rate either experienced mental effort or task difficulty. The scales were presented in random order. Each scale was preceded by a rest period of $8000 \mathrm{~ms}$ and an instruction screen of $3000 \mathrm{~ms}$, which primed subjects on which of the two VAS would be presented next. Following the instruction screen, the scales were presented for $10000 \mathrm{~ms}$. During this period, subjects could move a cursor on the scale and press a button to make a rating. Rating periods were kept at this standard duration to encourage actual rating instead of clicking on as fast as possible. For the subsequent analysis, only the actual time of rating up until the button press would be defined as the rating period in the fMRI protocol.

We employed the 150-point Rating Scale Mental Effort (RSME; Zijlstra, 1993) to allow subjects to assess the amount of invested mental effort they experienced. To assess difficulty, we designed a simple 150-point scale with three anchor points, following the argumentation of Yeo and Neal (2004) that visual scales used in the same experiment should be similar in design and scaling. The RSME is a vertical one-dimensional visual analogue scale (VAS), ranging from 0 to 150, with nine anchor points describing various levels of effort. These labels were carefully chosen and placed on the scale to ensure they have the same meaning for different subjects. The RSME assesses the combined regulative demands that are experienced by a subject as a result of both task load and state load. It is similar to other, routinely used instruments in measuring the construct of mental effort and is commonly used in the field of work psychology (Verwey \& Veltman, 1996). The reliability of the RSME is given in the literature with $r=.78$ (Zijlstra, 1993). This apparently low value is explained by the author as resulting from the fact that, even under laboratory conditions, the subjectively experienced amount of effort will vary over time even in the same subject. Previous research has demonstrated that the RSME does not rely on obvious cues to indicate differences in levels of mental work load (Zijlstra, 1993). Therefore it is unlikely that the obviousness of the differences between the conditions determines the resulting RSME scores. These obvious changes in task load were necessary for the evaluation of task difficulty.

The task and the two VAS were programmed in E-Prime. They were presented using E-Studio on a Windows XP PC connected to a MRI compatible optic system consisting of a projector and mirror goggles. Task and rating input was collected via an MRI compatible optical 2-button Joystick (Current Designs Inc., Philadelphia, USA). Subjects trained the handling of the Joystick for a brief period before the experiment by marking values on a VAS analogue to the ones used in the actual experiment.

Magnetic resonance imaging was performed on a Siemens Allegra 3T head scanner (Siemens AG, Erlangen, Germany) at the facilities of the Maastricht Brain Imaging Center. 
Anatomical imaging was carried out with a standard ADNI T1 weighted sequence, Voxel size 1 cubic $\mathrm{mm}$; flip angle $=9 \mathrm{deg}$; $\mathrm{TR}=2250 \mathrm{~ms}$; $\mathrm{TE}=2,6 \mathrm{~ms}$

Whole brain Echo-Planar Imaging (EPI) was performed using the following parameters: Matrix size 64x64; slice thickness 3,5mm; Slice order descending and interleaved; no gap; FOV 224x224mm; TE=30ms; TR=2000ms. Slice orientation was tilted 30 degrees backwards in order to minimize susceptibility artifacts in the orbitofrontal regions (Deichmann, Gottfried, Hutton, \& Turner, 2003)

\subsubsection{Analysis}

Behavioral data was analyzed using SPSS 15.0. A mixed model analysis was carried out in order to reflect the nested structure of the data. In order to check for exhaustion effects, the number of the session (1 or 2) was also included as a covariance factor.

\subsubsection{Imaging data treatment}

Analysis of fMRI data was performed in BrainVoyager QX 2.1 (Brain Innovation BV, Maastricht, The Netherlands). Anatomical images were individually preprocessed by inhomogeneity correction and extracranial noise filtering. The data was subsequently transformed into stereotactic space (Talairach \& Tournoux, 1988).The transformed anatomical scans from all subjects were then averaged into a single anatomical data set used as background for the visualization of group analyses.

The first three volumes of the functional scans were discarded because of magnetic saturation effects. The functional scans were preprocessed by slice scan time correction, motion correction and high pass filtering. Data of two subjects showed translation/rotation exceeding $3 \mathrm{~mm} / \mathrm{deg}$. Those datasets were excluded from further analysis. High pass filtering was performed using a General Linear Model (GLM) approach with a Fourier basis set which was adjusted to subtract the time course for predictors with up to 2 sine/cosine cycles per run and eventual linear trends from the time course of the data. Volume Time Course (VTC) files were calculated for each separate run.

\subsubsection{Statistical data analysis}

The E-Primer script for BrainVoyager (Hester Breman, Brain Innovation B. V., 2009) was used to extract the timing information of the single conditions from the E-Prime protocol files for each separate run. This timing information was used to build a design matrix. The single boxcar predictor time courses were adjusted for the shape and delay of the hemodynamic response by convolving them with a twogamma-function (Friston et al., 1998). Predictors for the translation/rotation of the subject's head were derived during the motion correction of the functional data and added in the design matrix. All predictors were z-transformed.

A random effects (RFX) GLM was computed for all runs of all subjects. For explorative analysis, the difficulty rating and the RSME rating condition were con- 
trasted with the baseline. To explore the specific effect of the effort-related RSME evaluation, both ratings were contrasted against each other. The resulting activation map was adjusted to a single-voxel threshold of $t=3.13(p<0.0096)$. This map was subsequently corrected for multiple comparisons by using the Cluster Threshold estimation plugin of BrainVoyager. This plugin runs a Monte-Carlo-Simulation extension (Forman et al., 1995) in order to determine the minimal cluster size given a user-defined confidence level, which was set to alpha $=.05$. The minimal size for the current data was calculated to be 11 contiguous voxels.

Supra-threshold clusters of active voxels were labeled using a microatlas of the human brain (Mai, Voss, \& Paxinos, 2007) and the Talairach daemon applet (Research Imaging Center, Texas, USA). Corresponding anatomical locations and approximate Broadmann areas (where applicable) were identified for each cluster.

\subsection{Results}

All subjects were able to perform the task at a sufficient level (mean score 18.29 correct out of 20 trials, $S D=0.91$ ). A sufficient level was defined as 15 correct responses out of 20 trials.

Mean RSME scores for the single n-back conditions were 27.99 (Stdev $=20.68)$, $42.91($ Stdev $=18.98)$ and $54.94($ Stdev $=20.71)$, for the 1-back, 2-back and 3-back, respectively. The according mean difficulty rating scores were 25.78 (Stdev = 23.81), 50.16 (Stdev = 24.92) and 66.65 (Stdev = 26.56) (see fig. 1). There was a significant correlation $(\mathrm{r}=.88, \mathrm{p}<.01)$ between the difficulty and RSME scores variables. There was no significant interaction effect between condition and the number of the session. Session did not have a significant main effect, either. The only significant main effect was elicited by condition: Pairwise comparisons of subject's evaluation scores on both the RSME and the difficulty scale showed significant differences $(\mathrm{p}<.01)$ between the conditions, confirming that the different n-back conditions indeed differed in perceived workload in a parametric fashion. This pattern could be expected from the original validation research of the RSME (Zijlstra, 1993) 


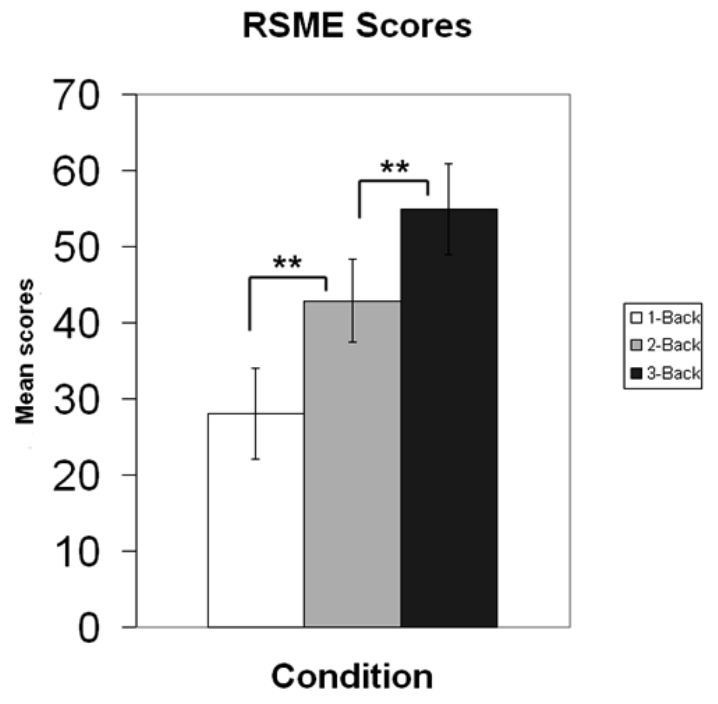

Fig. 1: Effort scores across different n-back conditions, illustrating the influence of task load on perceived effort. ${ }^{* *}=\mathrm{p}<.01$

As expected, the rating procedures as compared to baseline activated a large range of areas. Additionally to visual and motor areas, a number of working memory related areas in DLPFC and PPC became active during both rating tasks. Also, large clusters in the dorsal part of the mid-cingulate gyrus and in the anterior and posterior insula were activated. Furthermore, the inferior frontal gyrus (IFG) was activated. Various parts of the basal ganglia were activated, with strong bilateral thalamic activation present in both tasks. Bilateral activity was also found in the border region of external globus pallidus (GPe) and putamen, albeit localized slightly more lateral in the difficulty rating condition.

\begin{tabular}{lcccccl}
\hline Region & Peak X & Peak Y & Peak Z & n (vox) & \multicolumn{1}{l}{ t } & \multicolumn{1}{l}{$p$} \\
\hline Operculum & -46 & 25 & 0 & 214 & 4,86 & 0,000504 \\
Anterior Insula & -34 & 25 & 3 & 258 & 5,74 & 0,000131 \\
Precentral Gyrus & -52 & -5 & 42 & 417 & 7,32 & 0,000015 \\
Thalamus & -22 & -23 & -3 & 396 & 8,30 & 0,000005 \\
Superior Temporal Sulcus & -64 & -26 & 6 & 1085 & 6,63 & 0,000037 \\
Middle occipital gyrus & 5 & -74 & -6 & 9351 & 11,57 & 0,000000 \\
\hline
\end{tabular}

Fig. 2: Significantly more active clusters and their approximate locations in a contrast of mental effort vs. difficulty rating. P-values represent the peak p-value of a cluster.

A contrast of the two rating conditions showed clusters of significantly stronger activation in the left aIC and IFG/operculum, as well as in the thalamus. Also, stronger activation was seen in the right inferior parietal sulcus (IPS), bilateral occipital gyrus and in the left superior temporal sulcus (STS). Separate fixed effects group analysis of the two left handed subjects did not reveal any results that would 
point towards differences in the lateralization of the reported clusters due to handedness.

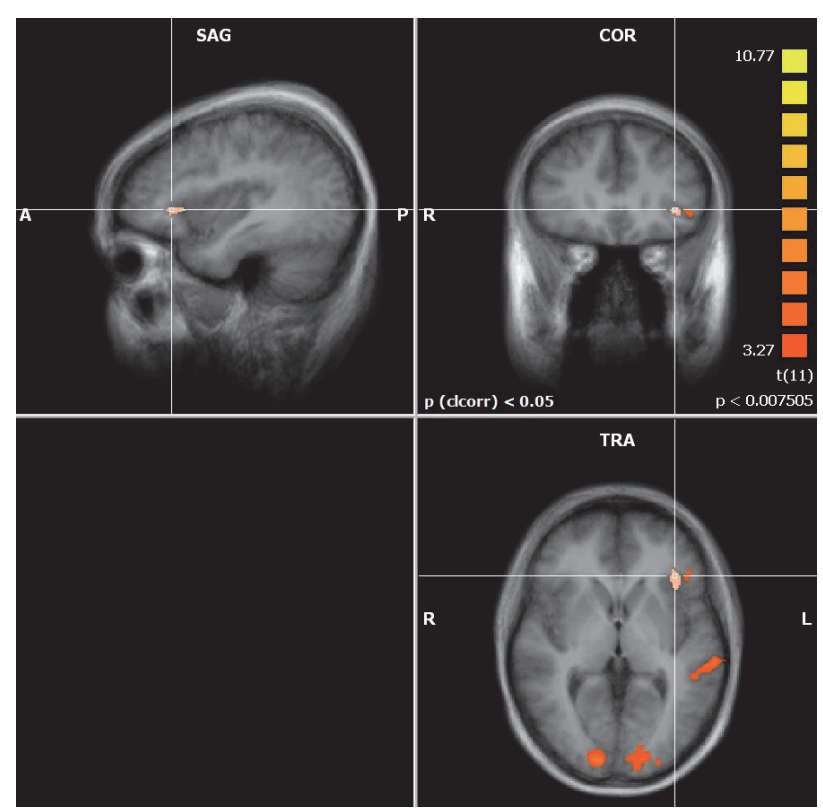

left AIC

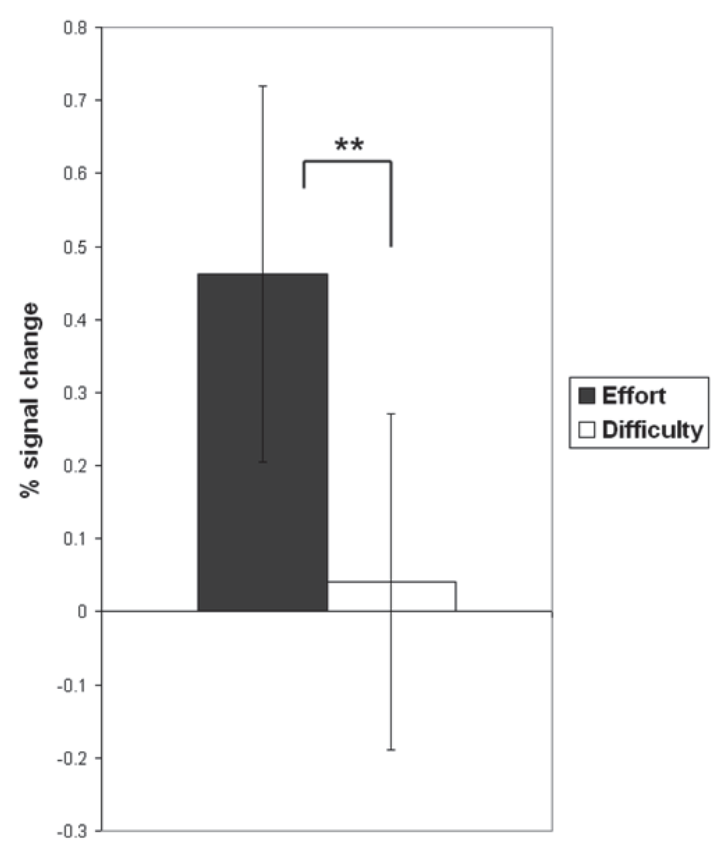

Fig. 3: Activation map showing the contrast mental effort rating $>$ difficulty rating

Fig. 4: Bar plot showing percent signal change during mental effort and difficulty rating in left aIC. ${ }^{* *}=p<.01$ 


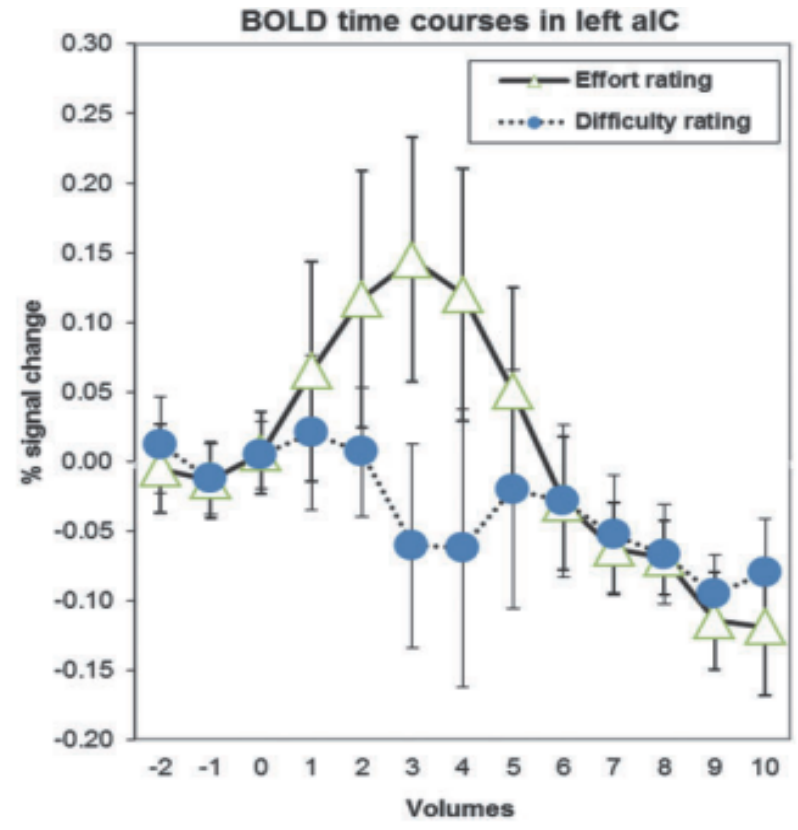

lalC Cluster during task execution

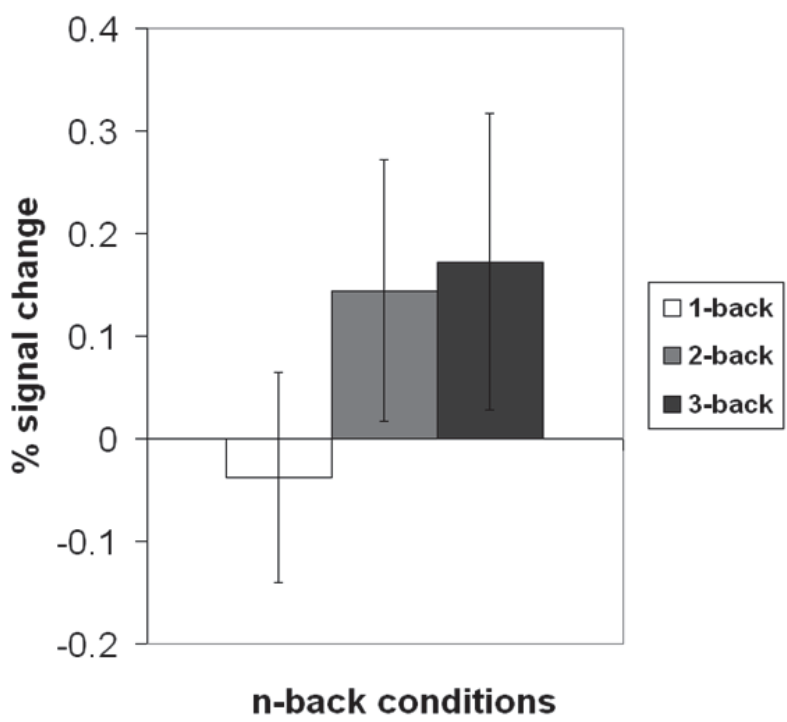

Fig. 5: BOLD curves showing percent signal change in left aIC during mental effort and difficulty rating, relative to the start of the actual rating period.

Fig. 6: Signal changes in left aIC during the execution of the $n$ back task. See also fig. 1. 


\subsection{Discussion}

\subsubsection{Summary of the findings}

We conducted this study to identify areas in the human brain that are relevant for the evaluation of mental effort. In particular, we investigated which areas would be significantly more active during mental effort evaluation when compared with a highly similar evaluation of task difficulty.

Our behavioral results confirmed our first hypothesis, regarding the correlation of difficulty and effort ratings and the induced differences in task load. As shown in earlier studies (Yeo \& Neal, 2004), difficulty and effort ratings were correlated. Also, the scores followed the induced differences in a parametric fashion, illustrating the effectiveness of our task load manipulation (fig. 1).

The brain imaging results for both rating tasks were in line with our second hypothesis. Common activation in both rating tasks included generally expected areas recruited for the basic execution of the VAS rating task, namely various visual and motor areas. Common activation also included anterior and posterior cingulate areas and dIPFC, i.e. results were very similar as those reported in several studies reviewed by Lee and Siegle (2009). During the rating task we furthermore observed increased activation in parts of the basal ganglia, as can be expected for tasks involving motor output as response.

\subsubsection{Activation in aIC}

Our main hypothesis concerned the contrast of the two rating task conditions. This hypothesis was confirmed: we found a cluster of significantly stronger activated voxels in the left aIC during mental effort evaluation (fig. $2 \& 3$ ). The aIC has been proposed to combine two necessary capabilities for the evaluation of mental effort: integrating information from different domains and making the result accessible to the subject as a part of self-awareness (Craig, 2009). This is in line with our findings.

We interpret the stronger activation in the left aIC in our subjects as evidence that mental effort evaluation indeed relies on this integrative process, compared to a more rational evaluation such as one of task difficulty.

The proposed function of aIC in self-awareness could also be related to the activation pattern we found. While both the assessments of difficulty and effort incorporate a cognitive appraisal of the task itself, mental effort evaluation additionally relies on emotional and somatic self-related factors. This difference in the self-referential aspect of our two evaluations resulted in a difference of aIC activation during the rating conditions. Similar results have been found in a study by Modinos et al. (2009), who investigated the neural correlates of self-evaluation. They employed a paradigm contrasting self-referential versus non-self-referential 
evaluation. Subjects had to evaluate the accuracy of various statements in relation to themselves or in relation to peers. The results of their study showed a cluster of activation in the left aIC, closely matching the location of the active cluster in our results. The left aIC showed a significantly stronger response when subjects were evaluating statements related to themselves contrasted with statements about peers. This was interpreted as evidence for the role of this region in self-related evaluations. In line with this evidence, we thus interpret the stronger activation in aIC found in our own results as reflecting the fact that mental effort evaluation relied more on the effect of the task on the state experienced by the subject during task performance. The contrast employed in our paradigm is, thus, more specific than the one used by Modinos et al. (2009), in the sense that it compared two selfcentered evaluations. This explains why we did not find differences in activation in medial prefrontal regions as Modinos et al. reported for a contrast of self- versus other-related evaluations.

An alternative explanation given by Modinos et al. (2009) was that self-related evaluation merely elicits enhanced awareness of somatic and emotional information reflected in aIC activation. This alternative interpretation is also relevant for the interpretation of our results, as it would imply that aIC is not actually recruited in the rating of mental effort, but merely becomes more activated by the resulting increase in self-awareness of self-interrogated somatic and emotional state. However, the results of Naccache et al. (2005) lend evidence against this interpretation. The disturbance in the somatic feedback loop left patient RMB bare of the capability to evaluate mental effort. If increased self-awareness was a mere by-product of the evaluative process, the process itself should not be impaired by a disturbance of the information streams related to self-awareness. Also, no changes were noted in patient RMB's general levels of self-awareness, demonstrating that other domains of self-referential processing which rely less on somatic information can still work normally in the light of this specific functional deficit. Our results support this interpretation, as our narrow experimental contrast of two self-centered evaluations did not elicit measurable differences in activation in the CMS, which is associated with differences in the level of general self-awareness. Our results illustrate the combination of processing capabilities of the aIC which are recruited significantly more during the evaluation of mental effort.

Additionally, our results suggest a more specific role of the left aIC connected to mental effort. When plotting the activation of the left aIC cluster during task execution, the activity varied in a monotonic fashion across the levels of task load, mirroring the pattern we observed in the corresponding RSME ratings (fig. 1). Similar observations have been reported earlier: Deary et al. (2004) found a change of activation in aIC as an effect of changing task difficulty, most prominent indeed in the left aIC. Although their design included no evaluations of task difficulty or mental effort, their behavioral results suggest effective changes in mental workload across the different levels of task difficulty. This indicates that in the case of mental effort, the left aIC has not only a role in offline evaluation, but also in experiencing mental 
effort during task execution. Shared insular regions that are recruited both during perceptual encoding of a self-related experience and during later evaluation with a VAS have been reported earlier (Kong et al., 2006).

Our findings are in line with evidence from these two studies, demonstrating that the experience of mental effort and later evaluations thereof share underlying neural structures.

\subsubsection{Other involved brain areas}

Additionally to the activation in left aIC, our maps showed a number of other areas that were stronger activated during mental effort evaluation than during task difficulty evaluation.

We interpret the activity in the left IFG / operculum as a result of the proposed strong connectivity between aIC and IFG / operculum (Craig, 2009). The activity in the left thalamus is in our view a sign of the heightened activity of the somatic loop, which in this case conveys information about bodily states to higher cognitive areas. The thalamic activation in our data was most prominent in the posterior ventral part of the thalamus. This part has been shown to project towards the aIC and the operculum. These projections have been proposed to play an important role in the awareness and evaluation of bodily states (Craig, 2002).

We also found a substantial cluster of stronger activated voxels in the left STS. Modinos et al. also reported a cluster in the superior temporal region, albeit contralateral to our results, in the right superior temporal gyrus (STG).

Their interpretation is that self-evaluation includes the estimation of what others might think about one. The STG has been reported to play a role in such theoryof-mind-related processes (Frith \& Frith, 2006).

Although we cannot rule out a similar explanation for our findings, our own interpretation is that the activation in STS is not related to a social feature of mental effort evaluation. The superior temporal area, especially the STS, is a functionally diverse region. It has been proposed that it might actually not be divided in specialized functional subregions, but that it provides a supportive role for different cognitive processes (Hein \& Knight, 2008). Our interpretation of the activation in the superior temporal region in our results is thus that it provided a supportive role. Differences in the task difficulty VAS and the RSME might have accounted for part of the reported clusters in our results as well; the difficulty scale did not use the eleven anchor points of the RSME, but merely three indications of difficulty. This already resulted in a richer visual stimulation, which we attribute as the cause of the more active clusters in the occipital cortex. The effects of the more precise scaling of the RSME might have affected the rating as such as well. The subjects might have tried to place their rating more precisely on a designated point on the RSME, compared with placing it in a general area on the difficulty scale. This would explain the stronger recruitment of the primary motor cortex, but also of the IPS as an area 
which has been shown to be essential for numerical distance processing (Ansari, Garcia, Lucas, Hamon, \& Dhital, 2005).

\subsubsection{Conclusion}

The goal of this study was to investigate the neural correlates of mental effort evaluation. By employing the specific contrast between the objective evaluation of task difficulty and the subjective evaluation of mental effort, we were able to identify a number of brain areas that partake stronger in this process. The relation of mental effort evaluation with somatic awareness had been proposed earlier on the basis of the observations in patient RMB (Naccache et al., 2005). These findings, together with the theoretical implications of the integrated model of mental effort regarding integration and awareness, made an involvement of the aIC plausible. Our results support the view that the aIC unifies two important capabilities necessary for mental effort evaluation. This, together with the stronger activation in the left thalamus, demonstrates the importance of somatic information for the emotionally salient awareness and evaluation of the self.

The relation between the RSME scores and the activation in left aIC during task performance suggests further research in this direction. Future studies could investigate the effect of various manipulations of mental workload on this area, which would solve the question if the apparent similarity originates in changes of task load or total perceived mental workload. Manipulating the state load, a variable which remained constant in the present study, could help to attribute these effects. 


\section{References}

Ansari, D., Garcia, N., Lucas, E., Hamon, K., \& Dhital, B. (2005). Neural correlates of symbolic number processing in children and adults. Neuroreport, 16(16), 1769.

Craig, A. D. (2002). How do you feel? Interoception: the sense of the physiological condition of the body. Nature Reviews Neuroscience, 3(8), 655-666.

Craig, A. D. (2009). How do you feel - now? The anterior insula and human awareness. Nature Reviews Neuroscience, 10(1), 59-70.

Damasio, A. (1999). The feeling of what happens. New York: Harcourt Brace \& Co.

Deary, I. J., Simonotto, E., Meyer, M., Marshall, A., Marshall, I., Goddard, N., \& Wardlaw, J. M. (2004). The functional anatomy of inspection time: an event-related fMRI study. Neuroimage, 22(4), 1466-1479.

Deichmann, R., Gottfried, J. A., Hutton, C., \& Turner, R. (2003). Optimized EPI for fMRI studies of the orbitofrontal cortex. NeuroImage, 19(2), 430-441.

Fairclough, S. H., \& Mulder, L. M. J. (2011). Psychophysiological Processes of Mental Effort Investment. In R. Wright \& G. Gendolla (Eds.), How Motivation Affects the Cardiovascular Response: mechanisms and applications. Washington DC: American Psychological Association.

Forman, S. D., Cohen, J. D., Fitzgerald, M., Eddy, W. F., Mintun, M. A., \& Noll, D. C. (1995). Improved Assessment of Significant Activation in Functional Magnetic Resonance Imaging (fMRI): Use of a Cluster-Size Threshold. Magnetic Resonance in Medicine, 33(5), 636-647. doi:10.1002/mrm.1910330508

Friston, K. J., Fletcher, P., Josephs, O., Holmes, A., Rugg, M. D., \& Turner, R. (1998). Event-Related fMRI: Characterizing Differential Responses. NeuroImage, 7(1), 30-40. doi:doi: DOI: 10.1006/nimg.1997 .0306

Frith, C. D., \& Frith, U. (2006). The Neural Basis of Mentalizing. Neuron, 50(4), 531-534. doi:doi: DOI: 10.1016/j.neuron.2006.05.001

Gevins, A., \& Cutillo, B. (1993). Spatiotemporal dynamics of component processes in human working memory. Electroencephalography and clinical Neurophysiology, 87(3), 128-143.

Hein, G., \& Knight, R. T. (2008). Superior temporal sulcus-It's my area: Or is it? Journal of Cognitive Neuroscience, 20(12), 2125-2136. doi:10.1162/jocn.2008.20148

Hockey, G. R. J. (1997). Compensatory control in the regulation of human performance under stress and high workload: A cognitive-energetical framework. Biological Psychology, 45(1-3), 73-93. doi: 10.1016/S0301-0511(96)05223-4

Kong, J., White, N. S., Kwong, K. K., Vangel, M. G., Rosman, I. S., Gracely, R. H., \& Gollub, R. L. (2006). Using fMRI to dissociate sensory encoding from cognitive evaluation of heat pain intensity. Human Brain Mapping, 27(9), 715-721. doi:10.1002/hbm.20213

Lee, K. H., \& Siegle, G. J. (2009). Common and distinct brain networks underlying explicit emotional evaluation: a meta-analytic study. Social Cognitive and Affective Neuroscience. doi:10.1093/scan/nsp001

Lieberman, M. D. (2010). Social cognitive neuroscience. In S. T. Fiske, D. T. Gilbert, \& Lindzey, G. (Eds.), Handbook of Social Psychology (5th ed., pp. 143-193). New York: McGraw-Hill. Retrieved from http://onlinelibrary.wiley.com/mrw_content/socpsy/articles/socpsy001005/socpsy001005.pdf

Mai, J. K., Voss, T., \& Paxinos, G. (2007). Atlas of the human brain (3rd ed.). Amsterdam, Boston: Elsevier/ Academic Press.

Modinos, G., Ormel, J., \& Aleman, A. (2009). Activation of Anterior Insula during Self-Reflection. (A. V. García, Ed.)PLoS ONE, 4(2), e4618. doi:10.1371/journal.pone.0004618

Mulder, G. (1986). The concept and measurement of mental effort. In G. R. J. Hockey, A. W. K. Gaillard, \& M. G. H. Coles (Eds.), Energetics and Human Information Processing. Dordrecht: Martinus Nijhoff.

Naccache, L., Dehaene, S., Cohen, L., Habert, M. O., Guichart-Gomez, E., Galanaud, D., \& Willer, J. C. (2005). Effortless control: executive attention and conscious feeling of mental effort are dissociable. Neuropsychologia, 43(9), 1318-1328.

Northoff, G., \& Bermpohl, F. (2004). Cortical midline structures and the self. Trends in Cognitive Sciences, 8(3), 102-107. doi:10.1016/j.tics.2004.01.004 
Owen, A. M., McMillan, K. M., Laird, A. R., \& Bullmore, E. (2005). N-back working memory paradigm: A meta-analysis of normative functional neuroimaging studies. Human Brain Mapping, 25(1), 46-59. doi:10.1002/hbm.20131

Paulus, M. P., \& Stein, M. B. (2006). An Insular View of Anxiety. Biological Psychiatry, 60(4), 383-387. doi:10.1016/j.biopsych.2006.03.042

Singer, T., Critchley, H. D., \& Preuschoff, K. (2009). A common role of insula in feelings, empathy and uncertainty. Trends in Cognitive Sciences, 13(8), 334-340. doi:10.1016/j.tics.2009.05.001

Talairach, J., \& Tournoux, P. (1988). Co-planar stereotaxic atlas of the human brain. New York: Thieme.

van der Meer, L., Costafreda, S., Aleman, A., \& David, A. S. (2010). Self-reflection and the brain: A theoretical review and meta-analysis of neuroimaging studies with implications for schizophrenia. Neuroscience \& Biobehavioral Reviews, 34(6), 935-946. doi:10.1016/j.neubiorev.2009.12.004

Verwey, W. B., \& Veltman, H. A. (1996). Detecting short periods of elevated workload: A comparison of nine workload assessment techniques. Journal of Experimental Psychology: Applied, 2(3), 270-285. doi:doi:10.1037/1076-898X.2.3.270

Yeo, G. B., \& Neal, A. (2004). A Multilevel Analysis of Effort, Practice, and Performance: Effects; of Ability, Conscientiousness, and Goal Orientation. Journal of Applied Psychology, 89(2), 231-247.

Zaki, J., Davis, J. I., \& Ochsner, K. N. (2012). Overlapping activity in anterior insula during interoception and emotional experience. NeuroImage, 62(1), 493-499. doi:10.1016/j.neuroimage.2012.05.012

Zijlstra, F. R. H. (1993). Efficiency in Work Behavior: A Design Approach for Modern Tools. Delft: Delft University Press. 


\section{Chapter 5 \\ Feeling the Force: Effort investment modulates activity in left anterior insular cortex}

Submitted as Otto, T., Zijlstra, F. R. H. \& Goebel, R. (2013). Feeling the Force: Effort investment modulates activity in left anterior insular cortex. 


\section{Abstract}

The left anterior Insular Cortex (laIC) has been shown to play a role in the selfevaluation of mental effort investment after performing a task. The amount of experienced effort investment related to carrying out a task depends on both task characteristics and the state of the person who carries out the task.

Earlier studies have also shown that laIC activation covariates with the task load during performance. The question remains if changes in laIC activation covariate only with task load, or rather with changes in experienced mental effort. In our study, we manipulate task as well as state load to identify brain areas which react to changes in experienced mental effort. 12 healthy participants performed an $n$ back task before and after either an exhausting or a free day while measuring brain activation with fMRI. Subjective mental effort was measured using the Rating Scale Mental Effort (RSME) and was found to be influenced by both task and state load changes, with an interaction of task and state effects on the exhaustive day. While bilateral anterior Insular Cortex (aIC) and several other areas reacted to changes in task load, only laIC also showed the same interaction effect that was present in the effort ratings. Available literature shows that laIC is involved in processes highly relevant to mental effort experience. This includes the representation of task rules and strategies, performance monitoring, and awareness of stressful stimuli. We propose that laIC unifies several functions which serve to manage effortful behavior. 


\subsection{Introduction}

\subsubsection{Scope of the study}

In the field of occupational psychology, the amount of effort an operator needs to invest to perform a task is a crucial factor in designing work places and interfaces. Mental effort investment refers to the energy invested in the task and is accompanied by a subjective feeling of strain, and its prolonged investment can pose a considerable stress on the organism. This, in turn, can lead to a depletion of resources, which can jeopardize further performance (Hockey, 1997). The amount of effort necessary for an individual to carry out a mental task is influenced by both the task difficulty and the individual's current state (Mulder, 1986). The subjectively experienced amount of mental effort can be measured using various instruments such as the one-dimensional Rating Scale Mental Effort (RSME; (Zijlstra, 1993). In order to measure all changes in mental effort investment, such instruments need to be sensitive to changes in the amount of effort experienced by the operator, and this ability has been confirmed in the original validation research of the RSME.

Next to rating scales, which can only be applied after task performance, various attempts have been made to also identify physiological indicators of acute mental effort investment. Such physiological indicators would enable the measurement of mental effort investment during task performance, without interrupting the task. Additionally, if a physiological indicator of mental effort reacts in the same way to changes in effort-inducing factors, this would provide evidence validating cognitive models of mental effort and the subjective self-report measurement instruments based on them.

Until now, research of such variables has targeted accessible, mostly peripheral variables, such as changes in cardiac functioning. Although certain components in these changes appeared to be promising (Mulder, 1986), more recent studies have demonstrated that these components lack the universal sensitivity necessary to map changes in mental effort expenditure (Veltman and Gaillard, 1996); (Nickel and Nachreiner, 2003). In the present study, we investigated the presence of indicators of mental effort investment directly in the brain. Such a direct approach has the advantage of eliminating the majority of distortions that might influence peripheral variables. A candidate for such an indicator would be the activity of the left anterior Insular Cortex (laIC). In an earlier study (Otto et al., 2012), we already identified this region as specifically involved during post-task self-evaluation of mental effort. Observations from this earlier study and a number of isolated findings from several other studies suggest an effort-dependent variation in laIC activation during task execution as well. In the current work, we describe the first coherent study into the reactivity of laIC to changes in mental effort. 


\subsubsection{Mental effort, state demands and task demands}

Performing a mental task, no matter how simple, requires regulative action from an individual. To ensure successful task completion, it takes monitoring and controlling of attention, information processing and behavior. The individual will also need to maintain a state of arousal that is suitable for performing the task. Together, all the regulative actions that an individual needs to perform are referred to as the mental workload. The integrated model of mental effort (Mulder, 1986) refers to two sources of demands: The task demands and the state demands. All regulative actions that are determined by the task itself, such as the need to control attention, information processing or responses, form the task demands. These can be varied by changes in task complexity, such as the number of items that need to be maintained in a working memory task. All factors that make it necessary for the individual to take regulative action in order to reach and maintain a psycho-physiological working state that allows task performance, however, form the state demands. Any mismatch of the actual and the required state of the individual, as for example in a situation where the individual is fatigued, increases the state demands,

The combination of these demands forms the total mental workload that a given task poses for an individual at a specific moment. In order to meet the mental workload, an individual needs to invest an according amount of mental effort. In the integrated model of mental effort, this is seen as a finite resource that fuels all regulative action. Mental effort is limited, thus it must be invested in a sustainable way.

\subsubsection{Measuring mental effort}

The fact that the amount of mental effort available to an individual is limited has important implications in the field of occupational psychology. On the one hand, in work environments where operator failure might entail critical consequences, it is vital to limit the total workload to an amount which is safely within the margin of the operator's resource. When designing such work environments, both the task complexity at every moment, but also the changes in the operator's state during the course of sustained performance need to be taken into account.

Investing effort as such is a willful action that results from the decision of an individual to accept a performance goal. Effort is invested to regulate all actions needed to reach the performance goal. At the same time, the investment of effort is experienced by the individual. The nature of this perception has been categorized as a quasi-emotional appraisal of one's own state (Damasio, 1999), meaning that one becomes conscious of how investing effort in a particular context influences one`s state.

This subjective experience of mental effort investment by an individual enables the use of self-report instruments to quantify the amount of mental effort that this individual needs to invest to perform a certain task. One-dimensional rating scales 
belong to the most commonly used and reliable self-report instruments that are employed in the field (Verwey and Veltman, 1996).

A point of concern remains in the fact that the concept of mental effort itself and the instruments constructed on its assumptions have been developed largely on the basis of behavioral evidence. The identification of objective variables that are sensitive to the same changes in demands would help to evaluate these assumptions and the derived instruments. Peripheral physiological variables were thoroughly investigated as indicators. One of the first and most promising variables was the $0.1 \mathrm{~Hz}$ heart rate variability (HRV) component (Mulder 1980). The evidence on the sensitivity of the $0.1 \mathrm{~Hz}$ component is inconclusive, though. More recently, it has been stated that the component might correspond rather to mental stress (Nickel and Nachreiner, 2003). While HRV and other peripheral variables are easily accessible, they only mediate the effect of mental effort expenditure in an indirect way. The possibility to infer a psychological variable from a physiological one depends on the specific connection between the two, and a causal relation is rarely found (Cacioppo and Tassinary, 1990). One of the exceptions is the relation of psychological processes and measures of brain functioning. Although this relation is also far from trivial, it can be used to gain fundamental insights into the neural correlates of cognitive concepts. As stated before, mental effort is a highly abstract concept. Finding a relation between the factors which have been found influential for it and measures of brain functioning would make it possible to validate and possibly refine the instruments used to measure it.

\subsubsection{The role of laIC in self-reports of mental effort investment}

In an earlier study (Otto et al., 2012), we already investigated which brain regions are specifically related to post-task self-evaluation of mental effort. Changes in regional cerebral blood flow (rCBF) were measured with functional Magnetic Resonance Imaging (fMRI) while participants performed short blocks of an n-back working memory task. The task had three levels of difficulty (1-, 2- and 3-back). After each block, the participants had to rate their experienced mental effort investment and the task difficulty on the RSME and on a separate visual analogue scale (VAS) modeled after the RSME. The contrast of subjective mental effort and task difficulty was chosen to compare similar task-related evaluations using similar instruments. A cluster of voxels in laIC showed increases in rCBF during mental effort evaluation compared to during difficulty evaluation. Yet, our results suggest a role of this area not only during post-task mental effort experience but also for mental effort experience, as the beta weights of the laIC cluster varied in a monotonic fashion over the levels of difficult during task performance.

This observation from our earlier study is in line with theoretical accounts and evidence from other studies: Brass and Haggard (2010) propose in a recent metaanalysis that the aIC provides an affective evaluation of the outcome of an intentional action, which serves as a guidance for sustainable, yet successful perfor- 
mance. The authors suggest that an increase in effort investment should be related to an increased recruitment of aIC, as the evaluation of the outcome of high-effortactions would be of increased importance for future decisions of effort investment. This view is corroborated by several observations of task-load-modulations of rCBF in aIC, most prominent on the left side (Deary et al., 2004); (Chee et al., 2004). These studies demonstrate evidence for a relation between task-related changes in workload and activation in laIC.

However, the amount of experienced mental effort expenditure depends not only on the task, but also on the state of the person. If laIC is indeed reactive to changes in experienced mental workload, this should also hold true under circumstances in which the workload varies as a result of differences in state, that is when a sub-optimal working state requires the operator to invest more effort to perform an unchanged task (Zijlstra, 1993); (Zijlstra, 1996).

There are few studies investigating the effects of state differences on task related brain activation in healthy participants. Mostly, state change is related to induction of fatigue. Examples of fatigue-inducing manipulations include sleep deprivation (Drummond et al., 2004), or administration of fatigue-inducing tryptophan (Morgan et al., 2006). Other examples of state change include recent (but not acute) consumption of cannabis versus sustained abstinence (Schweinsburg et al., 2010) or vaccination-related acute inflammation (Harrison et al., 2009). The results of these studies with respect to the aIC are inconsistent, and also the effects of the manipulation on experienced mental workload are either absent or not measured directly. Schweinsburg et al. (2010) investigated the effect of recent cannabis use versus sustained abstinence on fMRI - assessed brain activation during a spatial working memory (SWM) task and a vigilance control task. As expected, SWM task performance, compared to vigilance task performance, lead to an increase in activation in a number of cortical areas. The main finding, however, was that this increase was stronger for recent users compared to abstinent controls in several regions, including the bilateral aIC. This increase was more pronounced in laIC. While there was no direct measure of mental effort expenditure, there was a trend suggesting increased reaction time during the SWM task in recent users. It could be argued that previous cannabis use does not per se constitute a state difference, yet it forms a transient and reversible impairment to otherwise healthy brain functioning. This evidence indeed suggests a workload-related increased in aIC activity, albeit bilateral.

Harrison et al. (2009) employed the acute inflammation response due to vaccination together with a Stroop task featuring neutral and incongruent conditions. Two groups of participants received either a saline or a typhoid vaccine injection at two sessions. The inflammatory response to the vaccine caused a feeling of sickness, with increased fatigue and confusion in participants. During subsequent task performance while measuring changes in $\mathrm{rCBF}$ with fMRI, participants who received the vaccine showed increased activation in the bilateral aIC and mid-insular cortex, next to anterior and mid cingulate cortices (ACC/MCC), Thalamus, amygdala 
and the brainstem. The authors interpreted the absence of performance decrease in the vaccination condition as a sign of increased mental effort expenditure. This interpretation relies on the assumption that there was indeed an effect of treatment on mental workload. In that case, the findings of Harrison et al. would indeed suggest that changes in workload due to changes in state modulate insular activation. Yet, the absence of a measurement of this variable makes this impossible to test. Changes in state might be related to changes in related variables such as fatigue, but this does not automatically result in changes in mental workload. Drummond et al. (2004), for example, investigated a similar rationale with $35 \mathrm{~h}$ sleep deprivation versus normal sleep as experimental treatment, and a logical reasoning task at three levels of difficulty. While there was an effect of treatment on sleepiness as a state-related variable, there was no effect of treatment on perceived mental effort expenditure during task execution.

Summarizing, the current literature provides evidence which makes it seem plausible that state load - mediated changes in mental workload have an effect similar to that mediated by task load changes on aIC activation, possibly stronger on the left side. Yet, the absence of measures of mental effort expenditure in these studies does not allow for any conclusion in this question. Any brain region that is modulated by changes in mental workload has to be sensitive to changes in both task load and state load alike.

\subsubsection{Aims of the current study}

In the current study, we aimed to clarify the question if laIC is reactive to changes in subjectively experienced effort investment due to changes in total workload. We approached this question by employing a design featuring both changes in state and task load while monitoring changes in rCBF with fMRI and changes in subjective mental effort expenditure. Thereby, we aimed to identify brain regions which are sensitive to a change in perceived mental effort expenditure, resulting from changes in both state and task demands.

In order to manipulate state demands, we incorporated two different experimental treatments in our study. We chose to achieve changes in state by requiring participants to engage in sustained performance over the course of several hours, simulating the strain of a working day. Such long, effortful tasks have been successfully used to deplete the resources of participants, which leads to not only an increased feeling of mental fatigue, but also to higher costs for performing otherwise unchanged tasks (Scholey et al., 2009); (Boksem et al., 2005). To simulate a mentally exhausting day, participants absolved theoretical and practical helicopter takeoff training in the university's helicopter simulation lab. Similar tasks have been successfully employed to induce variations in state load reflected by decreases in vigilance and increases in drowsiness (Campagne et al., 2004) as well as, most important, increases in mental workload (Riese, 1999). The other treatment consisted 
of a day of self-chosen activities which explicitly did not incorporate any work, learning or other effortful activities.

Consequently, several hypotheses can be formulated:

\section{Hypothesis 1:}

Self-ratings of fatigue, well-being and emotional exhaustion will be influenced by the experimental day activity. Well-being will be reduced in the afternoon of the work day when compared to the afternoon of the free day. Fatigue and emotional exhaustion, however, will be increased.

\section{Hypothesis 2:}

The amount of subjectively experienced mental effort, measured with the RSME, will be influenced by the task conditions and the experimental day activities. Higher task load and higher state load will lead to increased scores on the RSME. The day activity is logically expected to influence the ratings only after the respective days.

Variations in the task load on working memory tasks affect rCBF in a number of cortical and subcortical regions. Ragland et al. (2002) showed an increase in rCBF due to increasing n-back task load in the bilateral insula, in several foci in the dorsolateral prefrontal cortex (DLPFC), the right anterior cingulate cortex (ACC) , bilateral inferior parietal cortex and in the left thalamus. We therefore expect to find an increase of rCBF in these regions due to increasing task load.

\section{Hypothesis 3:}

Increased task demands during n-back-task performance will be reflected in increased BOLD signal in the bilateral insula, DLPFC, right ACC, bilateral inferior parietal cortex and in the left thalamus.

The main research interest of this study is to combine changes in task- and state load in the same participants. Evidence from the literature leads us to expect that the activation in laIC will be modulated by changes in effort investment both due to changes in task load and due to changes in state load.

\section{Hypothesis 4:}

The BOLD signal in laIC will be influenced by the n-back - condition and the daycondition. Specifically, higher task load and higher state load will lead to an increased BOLD signal. We expect that the BOLD signal will thus show the same pattern of modulation as the behavioral scores measured with the RSME. 


\subsection{Methods}

\subsubsection{Participants}

15 (fifteen) healthy participants were recruited from the student body at Maastricht University. [Mean age 24.1 years, 6 male,]

Participants were invited only after being screened for any condition that would exclude participation in MRI research.

MRI - naïve participants were invited to participate in the piloting scans in the planning phase of the experiment. This was done in order to minimize the effect of the unfamiliar and potentially stressful MRI environment on participants, as we assumed that this would interfere with the effects of our experimental manipulation.

All participants were introduced to the working memory task and the rating interface before the start of the experiment. Informed consent was acquired from all participants before participation.

\subsubsection{Procedure}

Participants arrived at the facilities of the Maastricht Brain Imaging Center (MBIC) around 09:30h, (+- 1h). Previously, they were instructed to get their normal amount of sleep, and not to exceed moderate caffeine levels in the morning (maximum of two cups of coffee for habitual users not less than one hour preexperiment). The participants were then placed in the MRI scanner. Scanner-naïve participants were accustomed to the scanner 1-2 weeks prior to the experiment in order to reduce novelty effects or stress due to being exposed to the scanner for the first time.

While whole brain functional scans were acquired, participants performed a version of the n-back task (Gevins and Cutillo, 1993). The task consisted of 15 blocks of one minute each. Participants had to memorize letters appearing on a screen and indicate through a button press response if those letters were identical to the letter 1, 2, or 3 trials back. Each of the three conditions was presented five times, in a quasi-randomized order. Performance was measured as the number of correct button presses within the $2000 \mathrm{~ms}$ response window. Participants received feedback on each trial in order to be able to adjust their effort expenditure. After each one-minute block, participants rated their subjective expenditure of mental effort on the RSME (Zijlstra, 1993). The task and the RSME were programmed in EPrime (Psychology Software Tools, Inc., US). They were presented using E-Studio on a Windows XP PC connected to a MRI compatible optic system consisting of a projector and mirror goggles. Task and rating input was collected via an MRI compatible optical 2-button Joystick (Current Designs Inc., Philadelphia, USA). Partici- 
pants trained the handling of the Joystick for a brief period before the experiment by marking values on a VAS analogue to the one used in the actual experiment.

After this session, participants either underwent a $4 \mathrm{~h}$ training session in the university's helicopter cockpit mock-up or spend the same amount of time with self-chosen, low-effort activities. The training treatment was designed to induce the exhaustion level of a demanding work day. The helicopter training session consisted of a short theoretical instruction on helicopter take-off procedures and a practical part of trying to perform a takeoff procedure according to the presented guidelines. At around 16:00h, participants returned to the MRI lab. After again indicating their level of exhaustion on the two VAS, they were placed back in the scanner. The participants performed the same n-back paradigm as in the morning session. All subjects both underwent the free day treatment and the work day treatment in quasi-randomized order.

\subsubsection{Measurements}

In order to check for the effectiveness of the working day to induce a change in state, we had our participants provide self-ratings of exhaustion, fatigue and wellbeing. The degree of exhaustion was rated on two self-constructed 0-150 Visual Analogue Scales (VAS). On one VAS participants were asked to indicate their degree of tiredness, on the other their degree of being rested. Scores of the latter scale were reversed and a mean score of the two scales was calculated. Fatigue was assessed with four items from the Profiles of Mood Scale (POMS; McNair et al., 1971) as used for example by Sonnentag, Binnewies and Mojza (2008). Well-being was evaluated with four items from the revised English version of the Eigenzustands ("own state") scale (EZ-Scale) (Nitsch, 1976), as used by Sonnentag and Zijlstra (2006). All items were rated on five-point-scales. Fatigue and well-being have been demonstrated to be an estimate of experienced strain (Apenburg, 1986).

Mental effort expenditure was rated using the RSME (Zijlstra, 1993). Participants were able to use an optical joystick device to move the cursor along the scale in the fMRI scanner.

Magnetic resonance imaging was performed on a Siemens Allegra 3T head scanner (Siemens AG, Erlangen, Germany) at the facilities of the Maastricht Brain Imaging Center.

Anatomical imaging was carried out with a standard ADNI T1 weighted sequence, Voxel size1 cubic $\mathrm{mm}$; flip angle $=9 \mathrm{deg}$; $\mathrm{TR}=2250 \mathrm{~ms} ; \mathrm{TE}=2.6 \mathrm{~ms}$. Whole brain Echo-Planar Imaging (EPI) was performed using the following parameters: Matrix size 64x64; slice thickness 3,5mm; Slice order descending and interleaved; no gap; FOV 224x224mm; TE=30ms; TR= 2000ms. Slice orientation was tilted 30 degrees backwards in order to minimize susceptibility artifacts in the orbitofrontal regions (Deichmann et al., 2003) 


\subsubsection{Analysis of behavioral data}

Behavioral data was analyzed using SPSS 18. A mixed model analysis of the RSME scores was carried out in order to reflect the nested structure of the data. Factors included time-of-day (morning; T1, versus evening; T2); the day activity (working day, abbreviated WD, versus free day, abbreviated FD) the n-back condition (1-3) and the day order (first day versus second day). The day order was included in the model as, due to excluding a number of participants from the analysis for, the order of work day and free day was not balanced over the participants (4/12 starting with the free day). Reasons for exclusion consisted of misinterpretation of the rating instruments (1 case) and excessive movement during fMRI - measurements (two participants, criteria specified in section 5.2.5). The factors were centered and a model with interaction terms was build.

Well-being and fatigue items from each participant were pooled separately to form accumulated scores for the four respective time points (morning and afternoon of the working day and the free day). The accumulated scores were than compared using a paired-samples t-test, comparing the morning and afternoon scores per day and also the two afternoon scores. One subject reported a misinterpretation of the directionality on some of the items which lead to a number of missing values on the scales. Well-being and fatigue data from this subject was excluded from the behavioral analysis.

\subsubsection{Imaging data treatment}

Analysis of fMRI data was performed in BrainVoyager QX 2.3 (Brain Innovation BV, Maastricht, The Netherlands). Anatomical images were individually preprocessed by inhomogeneity correction and extracranial noise filtering. The data was subsequently transformed into stereotactic space (Talairach and Tournoux, 1988). The transformed anatomical scans from all subjects were then averaged into a single anatomical data set used as background for the visualization of group analyses. One participant was excluded as we found an anatomical abnormality. The participant was informed of this according to the guidelines set in the MBIC standard procedures.

The first three volumes of the functional scans were discarded because of magnetic saturation effects. The functional scans were preprocessed by slice scan time correction, motion correction and high pass filtering. Data of two subjects showed translation/rotation exceeding $3 \mathrm{~mm} / \mathrm{deg}$. Those datasets were excluded from further analysis. High pass filtering was performed using a General Linear Model (GLM) approach with a Fourier basis set which was adjusted to subtract the time course for predictors with up to 2 sine/cosine cycles per run and eventual linear trends from the time course of the data. Volume Time Course (VTC) files were calculated for each separate run. 


\subsubsection{Analysis of the imaging data}

The E-Primer script for BrainVoyager (Hester Breman, Brain Innovation bv., 2009) was used to extract the timing information of the single conditions from the EPrime protocol files for each separate run. This timing information was used to build a design matrix. The single boxcar predictor time courses were adjusted for the shape and delay of the hemodynamic response by convoluting them with a twogamma-function (Friston et al., 1998). Predictors for the translation/rotation of the subject's head were derived during the motion correction of the functional data and added in the design matrix.

A random effects (RFX) GLM was computed for the runs of all remaining 12 participants. To identify brain areas that react to changes in task load, we computed a contrast over all four measurement points between 1-back and 3-back task execution. The resulting activation map was adjusted to a single-voxel threshold of $\mathrm{t}=$ $4.18(\mathrm{p}<0.0015)$. As the RSME - scores revealed an interaction of task load and state load see results), we then proceeded to identify brain areas in which such a specific would also take place. Therefore, we computed an interaction contrast in which the difference between the difference of 1-back and 3-back in the morning and the difference of 1-back and 3- back in the afternoon of the working day was tested. We then subtracted the same difference calculated over the free day from this contrast in order to remain with voxels which would show the interaction effect stronger on the working day. This was done to correct for circadian effects, which we assumed to be equal on both days. The resulting activation map was adjusted to a single-voxel threshold of $t=3.36(\mathrm{p}<0.0064)$.

Both maps were subsequently corrected for multiple comparisons by using the Cluster Threshold estimation plugin of BrainVoyager. This plugin runs a MonteCarlo-Simulation extension (Forman et al., 1995) in order to determine the minimal cluster size given a user-defined confidence level, which was set to alpha $=.05$.

Locations of the remaining supra-threshold clusters of active voxels were localized using a microatlas of the human brain (Mai et al., 2007) and the Talairach daemon applet (Research Imaging Center, Texas, USA).

\subsection{Results}

\subsubsection{Task performance}

All participants were able to perform the task successfully (group mean 1.26 errors per 20 trials, group SD 1.55, minimum of .63 and maximum of 2.21 errors per 20 trials). 


\subsubsection{Self-reports}

One participant reported a misinterpretation of the scales for exhaustion and wellbeing. Data from this participant was excluded from this analysis. There was a clear effect of the day activity on self-reports of fatigue and well-being. Fatigue was rated higher in the afternoon of the working day than in the morning (mean / SD: 9.91 / 3.42 and $6.64 / 3.53 ; t(10)=-3.74, p<.05)$. It was also rated higher in the afternoon of the working day when compared to the afternoon of the free day (mean / SD: $9.91 / 3.42$ and $5.18 / 2.96 ; t(10)=5.84, p<.05)$. Fatigue was actually rated lower in the afternoon of the free day when compared to the morning of the free day (mean / SD: 5.18 / 2.96 and 6.45 / 3.36; $t(10)=-2,61, p<.05)$. Well-being was rated higher in the morning of the working day than in the afternoon (mean/SD: 16.45 / 3.05 and $13.64 / 4.23 ; t(10)=3.6, p<.05)$. There was no difference in well-being on the free day. Well-being was rated higher in the afternoon of the free day than in the afternoon of the working day (mean / SD: $17 / 2.05$ and 13.64 / 4.23; $t(10)=-3.39$, $p<.05)$. These results show that our state manipulation worked as predicted in hypothesis 1. Furthermore, fatigue was actually reduced over the course of the free day, which might indicate recovery from work stress unrelated to the experiment.

\subsubsection{Mental effort scores}

The mixed model analysis of the RSME scores showed several interaction effects. First, there was an interaction of time-of-day and the day activity $(F(1,177,94)=$ $4.81, p<.05)$. This was to be expected, as the effect of the state difference induction would be present after the respective day only. More important, we found an interaction of $n$-back condition with time-of-day $(F(1,733.92)=13.76, p<.05)$. This is in line with our expectations: The reported amount of mental effort expenditure is influenced by both the n-back condition and changes in state (which only happened on the evening of the working day), thus confirming hypothesis 2 . 


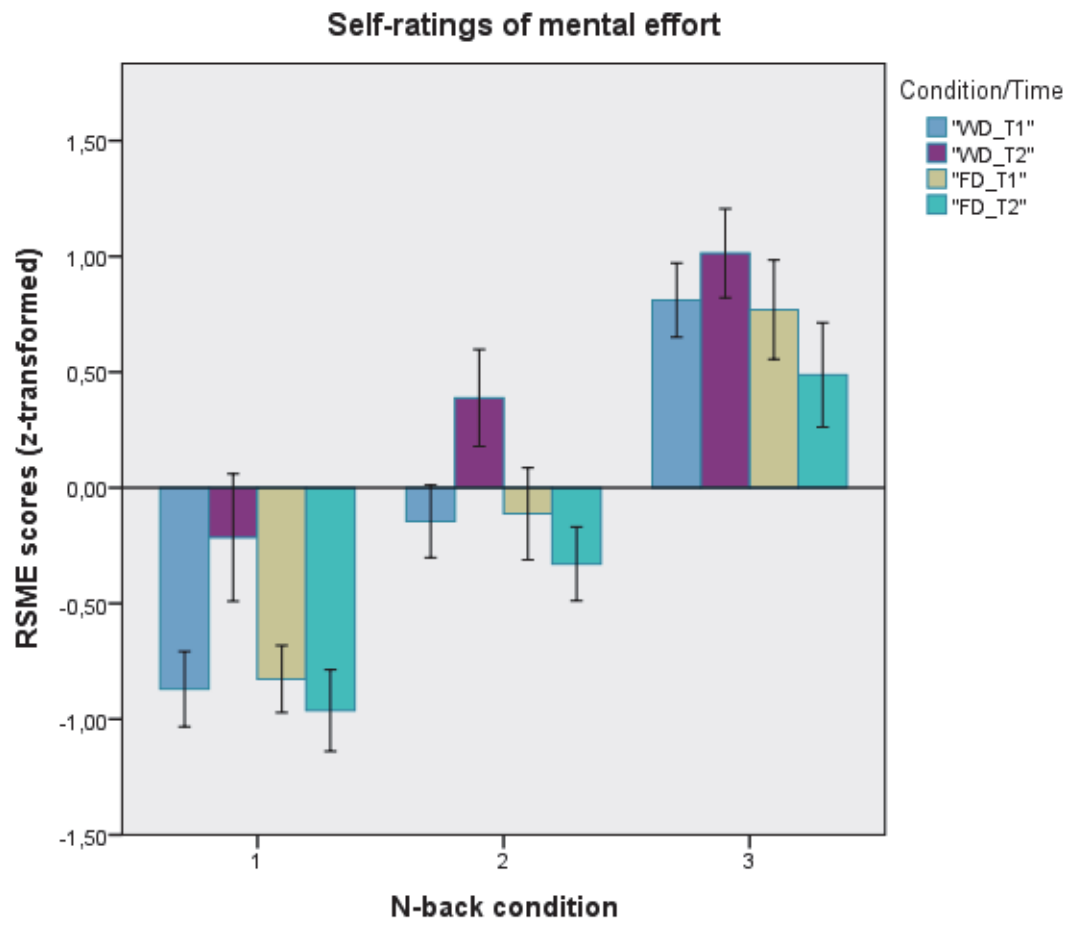

Error Bars: $95 . \% \mathrm{Cl}$

Fig. 1: Group means of the RSME scores over the four time points (Working Day (WD) and Free Day (FD), morning (T1) and evening (T2)) and the three n-back conditions. The effect of the three different n-back levels was influenced by the day activity at T2: At the evening of the work day (WD_T2), the three n-back conditions did not influence the experienced amount of mental effort as strongly, as even the most simple n-back condition required more regulative action. Increasing the task load of the n-back task lead to increased selfratings of mental effort investment. Also, the influence of changing state load due to the day treatment is clearly visible; at the evening of the work day, more effort was needed for the same task. Error bars represent $95 \%$ confidence intervals

\subsection{4. fMRI data}

The fMRI data revealed a robust effect of changes in task load over a wide range of brain areas. Contrasting 1-back versus 3-back, we found changes in rCBF in extended clusters in bilateral DLPFC/middle frontal gyrus (MFG), bilateral aIC, bilateral inferior parietal cortex, left thalamus and ACC ( $\mathrm{p}<.0015)$. Additionally, we found increased activity in the right thalamus, bilateral precentral sulcus and bilateral precuneus (Fig.2). Except for the latter differences, this replicates the results of Ragland et al. (2002) and in line with our third hypothesis. 

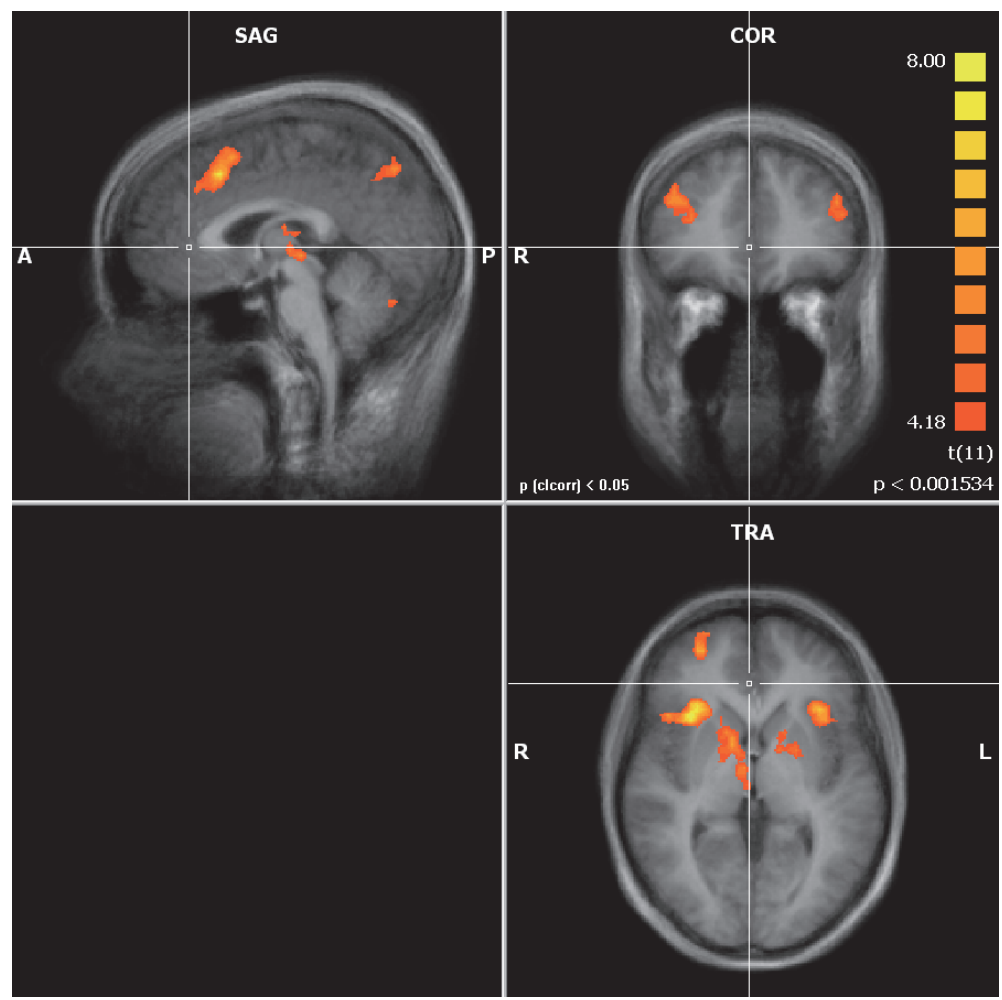

Fig. 2: Contrast showing 3-back > 1-back condition of the n-back task. Note the influence of increased task difficulty on bilateral aIC.

As a second step, we computed a contrast analogue to the interaction between task and state load we found in the mental effort scores.

This contrast represents the difference between the 1-back and the 3-back condition of the afternoon of the working day minus the difference between 1- and 3-back in the morning of the working day. Furthermore, we subtracted from this contrast the same contrast calculated over the free day. Thus, the map shows clusters of voxels in which there was a significant interaction between n-back-condition and time-of-day on the working day, corrected for the occurrence of such an effect on the free day.

Clusters that react in this way were found in laIC (218 voxels, center of gravity at Talairach coordinates $-31 ; 25 ; 7)$, at the border of the posterior medial superior frontal gyrus and the precentral gyrus (216 voxels, center of gravity at Talairach coordinates -3 ; -12 ; 57) and in the posterior cingulate cortex (PCC; 417 voxels, center of gravity at Talairach coordinates $-38 ;-17 ; 51$ ). From these clusters, only the one in laIC was also present in the contrast for task load. This is in line with hypothesis 4 . 

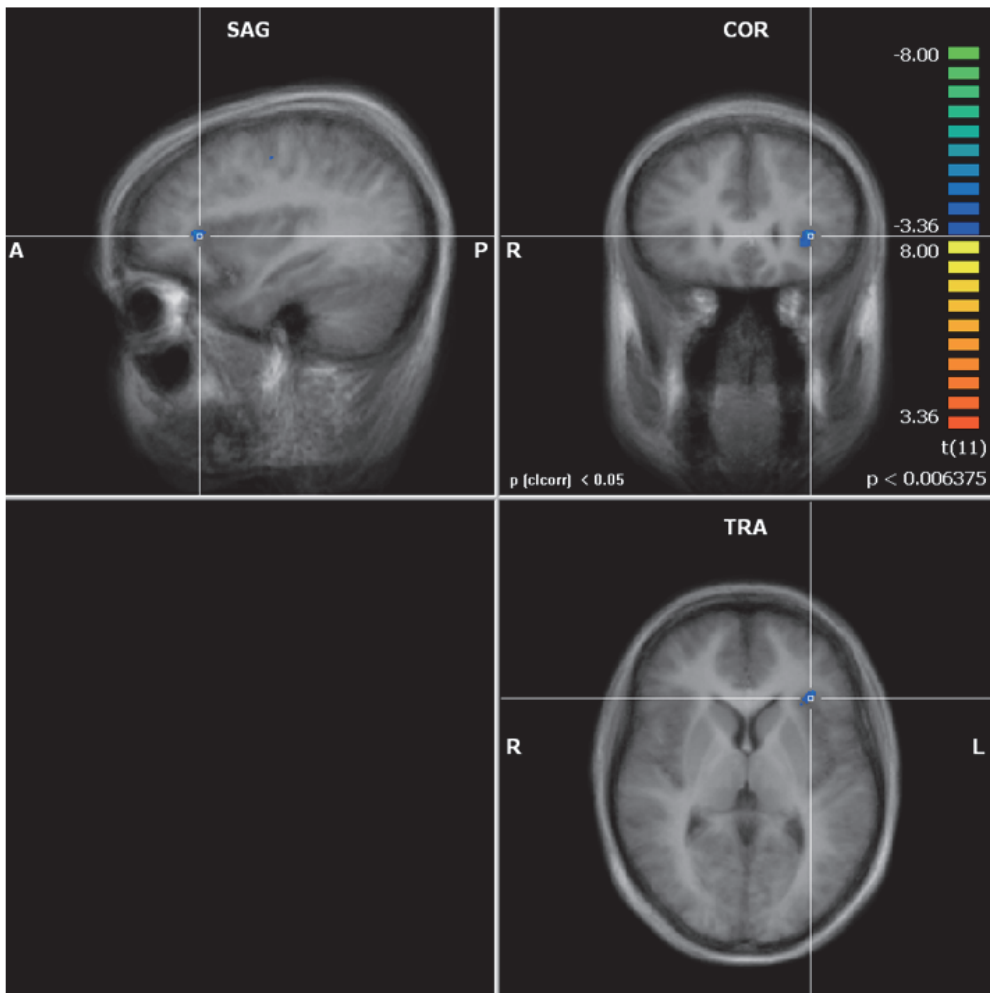

Fig. 3: A cluster in laIC showed the same interaction effect that was present in the RSME scores. The interaction of n-back difficulty*time-of-day*day activity leaves only clusters in which the effect of the n-back task was different in the afternoon of the working day compared to the morning of the working day, additionally controlled for a the occurrence of the same difference on the free day. 
Betas in lalC cluster

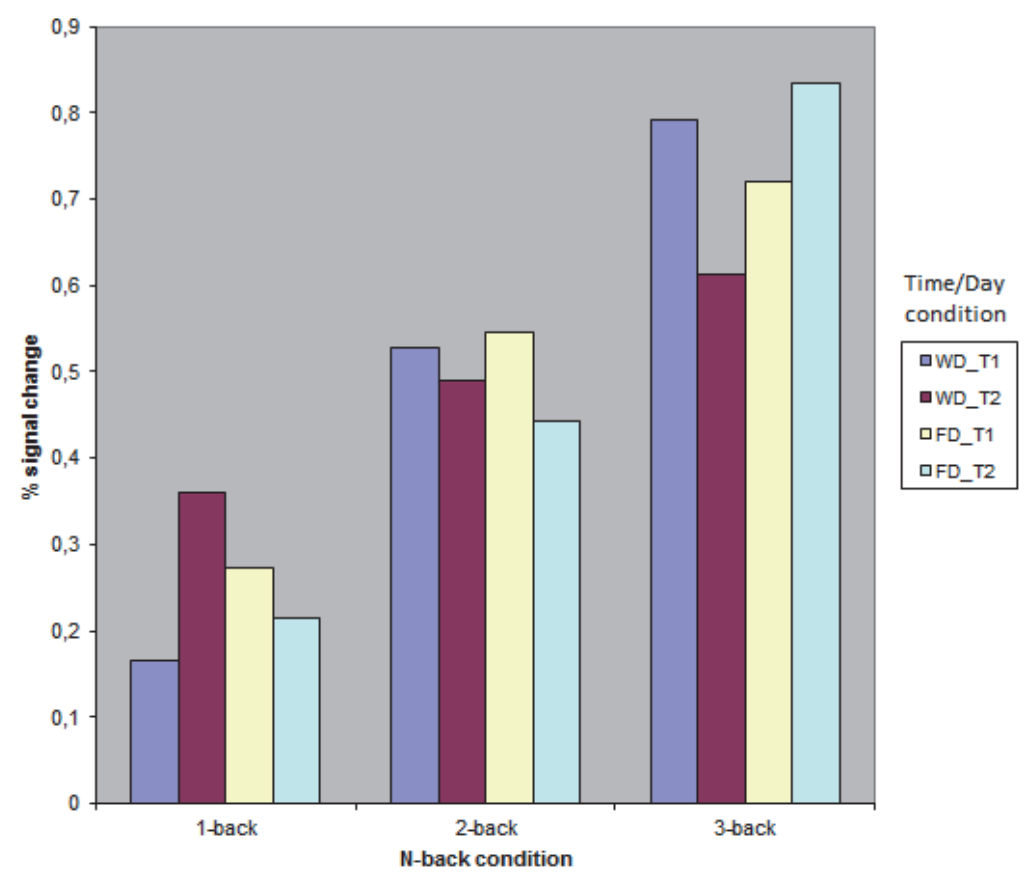

Fig. 4: Beta values of the laIC cluster. Note the reduction of the influence of changing n-back task load at the evening of the working day, WD T2.

\subsection{Discussion}

\subsubsection{Effectiveness of our experimental manipulation}

We conducted this study in order to identify brain areas which react to changes in mental workload caused by both changes in task load and state load. We were able to successfully induce said changes in experienced mental effort expenditure in our experiment. The different levels of the $n$-back task caused distinct levels of mental effort investment, as shown by the RSME ratings. Furthermore, spending several hours in an exhaustive learning task did induce state changes in our participants, as documented by increased fatigue and reduced well-being in the afternoon measurement. This state change cannot be attributed to circadian effects, as the comparison measurement on the free day did not show any reduction in well-being, and even revealed a decrease in fatigue.

The change to a suboptimal state resulted in changes to the amount of subjectively experienced mental effort expenditure in our participants. After the working day, participants reported increased mental effort expenditure compared to all 
other time points. Furthermore, the effect of the n-back conditions was reduced, as the state load consumed a more substantial portion of the available effort resources.

\subsubsection{Isolating neural correlates of mental effort}

At the same time, our analysis of the fMRI data revealed a similar effect of our experimental manipulations on $\mathrm{rCBF}$ in laIC. We first checked for differences occurring at the different levels of task load. As expected, there was indeed a stable effect of task load. This effect was not confined to the laIC, but included the right aIC as well as ACC, the DLPFC/MFG, and several parietal areas. This is in line with earlier research on the effect of different levels of difficulty in working memory tasks (Ragland et al., 2002). The main goal of this study, however, was to identify brain areas which also react to changes in the amount of subjective experienced mental effort mediated by changes in state load. When testing for the same specific interaction that was present in the behavioral data, a cluster of voxels in laIC displayed these effects. At this point we would like to re-iterate that the interaction contrast reported here was performed on the whole brain, without the use of any mask. Thus, the occurrence of this cluster is purely due to the fact that rCBF in these voxels was modulated by our experimental manipulation in the same way as our behavioral ratings. Such a relation between subjectively experienced amount of mental effort and the rCBF in a brain region is unique to our knowledge. In our interpretation, this evidence, together with the previously shown increased activation of laIC in mental effort evaluation, demonstrate the crucial role of this structure in monitoring the balance of effort output in the face of changing resources.

It is important to keep in mind that the concept of mental effort involves more than purely the perception of acute strain. Mental effort is a limited resource, and its sustainable management is vital for the functioning of an organism in a larger context (Hockey, 1997). A meaningful evaluation of effort investment must take the state of the organism into account (Mulder, 1986). Supporting this interpretation, several studies and meta-analyses provide evidence of aIC involvement in functions highly relevant to the concept of mental effort. The bilateral aIC, specifically the more anterior part, has been identified as part of a network underlying domaingeneral maintenance of task rules and strategies ((Dosenbach et al., 2008). Thus, aIC is involved in the representation of the actual task set, which defines the mental workload posed on the organism. Additionally, there is evidence that suggests that aIC also monitors ongoing task performance. Nelson et al. (2010) reported that in particular the anterior part of aIC is, additionally to top-down task control, also involved in integrating task-and performance-related bottom-up information. Performance monitoring is essential for the adjustment of effort output under changing workload conditions (Naccache et al., 2005).

In summary, these accounts converge on a picture in which the aIC is at a critical junction between the experience, evaluation and investment of mental effort. 
The left-sided lateralization of our aIC cluster does raise the question of a functional heterogeneity in aIC across hemispheres. A possible explanation is the reliance of the process of mental effort experience on certain somatic inputs. The experience of mental effort relies on integration of several sources of information about the self, and information about bodily states is thought to be crucial for this process (Naccache et al., 2005). In this context, (Gray et al., 2007) have proposed the laIC as a target site for heartbeat- evoked potentials (HEP). In their combined EEG/ECG study, Gray et al. contrasted a high-workload arithmetic task with a baseline counting task in patients with heart problems. The high-workload task proved to be more stressful than the control task. Individual stress-related changes in myocardial output were significantly correlated with changes in HEP amplitude in electrode sites close to laIC. The authors propose that laIC is the principal target site of afferent signals related to changes in myocardial function caused by changes in acute stress. Evidence from other studies supports this interpretation, as laIC activity has been found to be modulated also by other stressful stimuli such as increasing negative valence in visual stimuli (Anders et al., 2004). Another study by Wiech et al. (2010) found a modulation of bilateral aIC activation by increasing the expectancy of an imminent heat stimulus as being damaging in their participants. However, only laIC, and not its right counterpart, was found to increase functional connectivity with the midcingular gyrus, which in turn was activated more during subsequent stimulus application and predicted the perceived painfulness of the stimulus. Together, these results demonstrate that laIC serves a role in the propagation of the awareness for stressful conditions, not confined to a single modality or domain. Stress is not synonymous to high effort investment, but already Mulder (1986) noted the close relation between those concepts when proposing the use of myocardial measurements as indicators of mental effort. In our interpretation, mental effort experience is inherently the result of integrating different information sources about the state of the self, with information about myocardial functioning being a necessary, but not sufficient component.

\subsubsection{Limitations and future directions}

The slightly unbalanced order of free day and working day due to participant exclusions forms a minor concern, as a learning effect on the n-back task would not be completely independent from our day activity condition and hence might have affected our effort ratings. However, we repeated the analysis of the effort scores with a completely balanced sample of 6 participants starting with the free day $(2$ of which had previously been excluded from the fMRI analysis) and 6 randomly drawn participants starting with the work day. This analysis showed the same effects as the ones we report earlier, without the possibility of contamination due to learning effects.

Future investigations should solve two questions: First, the connectivity of laIC with other regions. Akin to the findings of Wiech et al. (2010), in the expectancy of 
painful stimuli, an increase in laIC activation might be paired with increased connectivity to other, more specific areas. The knowledge about such connections would be helpful in connecting mental effort investment to other, related processes, and thereby to understand mental effort in a more holistic context. Two specific processes of interest would be the motivational processes that precede effort investment, and the emotional consequences of effort investment in terms of reward.

Second, future studies could help to understand the relation of HEPs and laIC activation related to changes in mental workload. This would potentially lead to a more complete understanding of the systemic processes that surround mental effort investment, which might even clarify the role of other related variables such as the much-discussed $0.1 \mathrm{~Hz} \mathrm{HRV}$ component.

The present study is one of the first investigations into a concept originating in occupational psychology using neuroscientific methods. While other domains such as perception have seen great advances already, our understanding of the fundamental processes that take place during work is just starting to developed. The significance, however, is enormous: Work defines a large part of our waking adult life, and our interaction with the challenges posed by our occupation can have an influence on every other part of our lives (Paul, \& Batinic, 2009). Understanding how the brain meets these challenges and adapts to them will help to design work in such a way that this interaction is beneficial for the person carrying out the work. The current study and our earlier work are examples of this emerging "occupational neuroscience" in extending the rich knowledge of a predominantly cognitive discipline with the tools and methodological advances that are provided by neuroscience. 


\section{References}

Anders, S., Lotze, M., Erb, M., Grodd, W., Birbaumer, N., 2004. Brain activity underlying emotional valence and arousal: A response-related fMRI study. Hum. Brain Mapp. 23, 200-209.

Apenburg, E., 1986. Befindlichkeitsbeschreibung als Methode der Beanspruchungsmessung [Description of well-being as a method for measuring strain]. Psychologie und Praxis 30, 3-14.

Boksem, M.A.S., Meijman, T.F., Lorist, M.M., 2005. Effects of mental fatigue on attention: an ERP study. Cognitive Brain Res. 25, 107-116.

Brass, M., Haggard, P., 2010. The hidden side of intentional action: the role of the anterior insular cortex. Brain Struct. Funct. 214, 603-610.

Cacioppo, J.T., Tassinary, L.G., 1990. Inferring psychological significance from physiological signals. Am. Psychol. 45, 16-28.

Campagne, A., Pebayle, T., Muzet, A., 2004. Correlation between driving errors and vigilance level: influence of the driver's age. Physiol. Behav. 80, 515-524.

Chee, M.W.L., Soon, C.S., Lee, H.L., Pallier, C., 2004. Left insula activation: A marker for language attainment in bilinguals. Proceedings of the National Academy of Sciences of the United States of America 101,15265 .

Damasio, A., 1999. The feeling of what happens. Harcourt Brace \& Co, New York.

Deary, I.J., Simonotto, E., Meyer, M., Marshall, A., Marshall, I., Goddard, N., Wardlaw, J.M., 2004. The functional anatomy of inspection time: an event-related fMRI study. Neuroimage 22, 1466-1479.

Deichmann, R., Gottfried, J.A., Hutton, C., Turner, R., 2003. Optimized EPI for fMRI studies of the orbitofrontal cortex. Neuroimage 19, 430-441.

Dosenbach, N.U.F., Fair, D.A., Cohen, A.L., Schlaggar, B.L., Petersen, S.E., 2008. A dual-networks architecture of top-down control. Trends in Cog. Sci. 12, 99-105.

Drummond, S.P.A., Brown, G.G., Salamat, J.S., Gillin, J.C., others, 2004. Increasing task difficulty facilitates the cerebral compensatory response to total sleep deprivation. Sleep - New York then Westchester $27,445-452$.

Forman, S.D., Cohen, J.D., Fitzgerald, M., Eddy, W.F., Mintun, M.A., Noll, D.C., 1995. Improved Assessment of Significant Activation in Functional Magnetic Resonance Imaging (fMRI): Use of a Cluster-Size Threshold. Magn. Reson. Med. 33, 636-647.

Friston, K.J., Fletcher, P., Josephs, O., Holmes, A., Rugg, M.D., Turner, R., 1998. Event-Related fMRI: Characterizing Differential Responses. Neuroimage 7, 30-40.

Gevins, A., Cutillo, B., 1993. Spatiotemporal dynamics of component processes in human working memory. Electroencephalogr Clin Neurophysiol 87, 128-143.

Gray, M.A., Taggart, P., Sutton, P.M., Groves, D., Holdright, D.R., Bradbury, D., Brull, D., Critchley, H.D., 2007. A cortical potential reflecting cardiac function. Proc. Natl. Acad. Sci. 104, 6818.

Harrison, N.A., Brydon, L., Walker, C., Gray, M.A., Steptoe, A., Dolan, R.J., Critchley, H.D., 2009. Neural Origins of Human Sickness in Interoceptive Responses to Inflammation. Biol. Psychiat. 66, 415-422.

Hockey, G.R.J., 1997. Compensatory control in the regulation of human performance under stress and high workload: A cognitive-energetical framework. Biol. Psychol. 45, 73-93.

Mai, J.K., Voss, T., Paxinos, G., 2007. Atlas of the human brain, 3rd ed. Elsevier/ Academic Press, Amsterdam, Boston.

McNair, D.M., Lorr, M., Droppleman, L.F., 1971. Manual for the Profile of Mood States. Educational and Industrial Testing Services, San Diego, CA.

Morgan, R.M., Parry, A.M.M., Arida, R.M., Matthews, P.M., Davies, B., Castell, L.M., 2006. Effects of elevated plasma tryptophan on brain activation associated with the Stroop task. Psychopharmacology 190, 383-389.

Mulder, G., 1986. The concept and measurement of mental effort, in: Hockey, G.R.J., Gaillard, A.W.K., Coles, M.G.H. (Eds.), Energetics and Human Information Processing. Martinus Nijhoff, Dordrecht.

Naccache, L., Dehaene, S., Cohen, L., Habert, M.O., Guichart-Gomez, E., Galanaud, D., Willer, J.C., 2005. Effortless control: executive attention and conscious feeling of mental effort are dissociable. Neuropsychologia 43, 1318-1328. 
Nelson, S.M., Dosenbach, N.U.F., Cohen, A.L., Wheeler, M.E., Schlaggar, B.L., Petersen, S.E., 2010. Role of the anterior insula in task-level control and focal attention. Brain Struct. Funct. 214, 669-680.

Nickel, P., Nachreiner, F., 2003. Sensitivity and Diagnosticity of the 0.1-Hz Component of Heart Rate Variability as an Indicator of Mental Workload. Hum. Factors 45, 575-590.

Nitsch, J.R., 1976. Die Eigenzustandsskala (EZ-Skala) - Ein Verfahren zur hierarchischmehrdimensionalen Befindlichkeitsskalierung, in: Nitsch, J.R., Udris, I. (Eds.), Beanspruchung Im Sport. Limpert, Bad Homburg, pp. 81-102.

Otto, T., Zijlstra, F.R.H., Goebel, R., 2012. Neural correlates of mental effort evaluation - Involvement of structures related to self awareness. SCAN.

Ragland, J.D., Turetsky, B.I., Gur, R.C., Gunning-Dixon, F., Turner, T., Schroeder, L., Chan, R., Gur, R.E., 2002. Working memory for complex figures: An fMRI comparison of letter and fractal n-back tasks. Neuropsychology 16, 370.

Riese, H., 1999. Mental Fatigue after Very Severe Closed Head Injury: Sustained Performance, Mental Effort, and Distress at Two Levels of Workload in a Driving Simulator. Neuropsychol. Rehab. 9, 189205.

Scholey, A.B., French, S.J., Morris, P.J., Kennedy, D.O., Milne, A.L., Haskell, C.F., 2009. Consumption of cocoa flavanols results in acute improvements in mood and cognitive performance during sustained mental effort. J. of Psychopharmacology 24, 1505-1514.

Schweinsburg, A.D., Schweinsburg, B.C., Medina, K.L., McQueeny, T., Brown, S.A., Tapert, S.F., 2010. The Influence of Recency of Use on fMRI Response During Spatial Working Memory in Adolescent Marijuana Users. J. Psychoactive Drugs 42, 401-412.

Sonnentag, S., Binnewies, C., Mojza, E.J., 2008. “ Did you have a nice evening?” A day-level study on recovery experiences, sleep, and affect. J Appl Psychol 93, 674.

Sonnentag, S., Zijlstra, F.R.H., 2006. Job characteristics and off-job activities as predictors of need for recovery, well-being, and fatigue. J Appl Psychol 91, 330-350.

Talairach, J., Tournoux, P., 1988. Co-planar stereotaxic atlas of the human brain. Thieme, New York.

Veltman, J.A., Gaillard, A.W.K., 1996. Physiological indices of workload in a simulated flight task. Biol. Psychol. 42, 323-342.

Verwey, W.B., Veltman, H.A., 1996. Detecting short periods of elevated workload: A comparison of nine workload assessment techniques. J Exp Psychol Appl 2, 270-285.

Wiech, K., Lin, C., Brodersen, K.H., Bingel, U., Ploner, M., Tracey, I., 2010. Anterior insula integrates information about salience into perceptual decisions about pain. J. Neurosci. 30, 16324-16331.

Zijlstra, F.R.H., 1993. Efficiency in Work Behavior: A Design Approach for Modern Tools. Delft University Press, Delft.

Zijlstra, F.R.H., 1996. Effort as energy regulation., in: W. Battmann S. Dutke (Ed.), Processes of the Molar Regulation of Behavior. Pabst Science Publishers, Lengerich, Germany, pp. 219-235. 
Chapter 6

\section{Spatially distributed effects of mental exhaustion on Resting-State fMRI networks: An ICA-based analysis of the resting human brain after sustained cognitive performance}

Submitted as Esposito, F., Otto, T., Zijlstra, F. R. H., \& Goebel, R. (2013). Spatially distributed effects of mental exhaustion on functional MRI networks: An ICA-based analysis of the resting human brain after sustained cognitive performance. 


\section{Abstract}

Brain activity during rest is spatially coherent over functional connectivity networks called resting-state networks (RSNs). In resting-state functional magnetic resonance imaging (RS-fMRI), independent component analysis (ICA) yields spatially distributed RSN representations reflecting distinct mental processes, such as intrinsic (default) or extrinsic (executive) attention, and sensory inhibition or excitation. These aspects can be related to different treatments or subjective experiences. Among these, exhaustion is a common psychological state induced by prolonged mental performance. Using repeated RS-fMRI measurements and ICA, we explored the effect of several hours of sustained cognitive performances on the resting human brain.

RS-fMRI was repeated on healthy volunteers in two days, with and without, and before, during and after, an intensive psychological treatment (skill training and sustained practice with a flight simulator). After each scan, subjects rated their level of exhaustion and performed an N-back task. ICA maps from baseline scans were clustered within and between subjects, yielding RSN templates. Selected RSN components from all scans were then evaluated to detect any changes induced by the sustained mental performance.

The intensive treatment had a significant effect on exhaustion and effort ratings, but no effects on N-back performances. Significant changes in the most exhausted state were observed in the early visual processing and the anterior default mode networks (enhancement) and in the right and left fronto-parietal executive networks (suppression), suggesting that mental exhaustion is associated with a more idling brain state and that internal attention processes are facilitated to the detriment of more extrinsic processes. As the described application of ICA to RSfMRI has highlighted these effects before performance degradation, this technique may inspire future indicators of the level of fatigue in the neural attention system. 


\subsection{Introduction}

Functional magnetic resonance imaging (fMRI, Ogawa et al., 1990) in the absence of experimental tasks and behavioral responses, performed with the subject in a relaxed "resting state" (RS-fMRI) allows measuring the amount of spontaneous bloodoxygen-level-dependent (BOLD) signal synchronization within and between multiple regions across the entire brain (Biswal et al., 1995). In fact, RS-fMRI activity is characterized by low frequency $(0.01-0.1 \mathrm{~Hz})$ BOLD signal fluctuations, which are topologically organized as multiple spatially distributed functional connectivity networks called resting-state networks (RSNs) (van de Ven et al., 2004; Damoiseaux et al., 2006; De Luca et al., 2006; Mantini et al., 2007; van den Heuvel et al., 2009). As the RSN constituent regions partially or totally overlap with typical brain activations induced by perceptual and cognitive tasks (Gusnard and Raichle, 2001;Smith et al., 2009), the study of one or more RSNs has allowed a system-level functional description of several mental processes and the characterization of the associated brain status. More recently, by repeating RS-fMRI scans under different experimental conditions, it has been possible to relate these processes to (and manipulate by) externally modifiable factors, such as different pharmacological treatments or psychological experiences (Albert et al., 2009; Esposito et al., 2010; Khalili-Mahani et al., 2011).

Independent component analysis (ICA) (Hyvarinen et al., 2001), if applied to whole-brain fMRI time-series, allows decomposing a single data set into a series of activation images called ICA "components" with associated time-courses of activation, without the need to select a specific temporal profile or constrain the anatomical space of interest. While some of the ICA components describe artifacts and other noise sources in the data, other components describe the BOLD signal temporal correlations within and between functionally connected brain regions (McKeown et al., 1998). However, unlike "seed-based" functional connectivity analysis approaches, the spatial distribution of ICA components does not rely on targeting one or another brain region for the analysis. Moreover, in RS-fMRI, several ICA components correspond to highly reproducible RSNs (Damoiseaux et al., 2006; Mantini et al., 2007), suggesting how ICA can be used for both characterizing and mapping RSN functional connectivity in a region-independent fashion, and producing separate spatially distributed (voxel-level) representations of distinct mental processes, such as, e. g., attention and perception, in a task-independent fashion.

Exhaustion is a common psycho-physiological state after prolonged mental performance. It reflects an individual's need for substantial recovery that arises after sustained expenditure of mental effort to meet task demands (Sonnentag and Zijlstra, 2006). Exhaustion is also associated with an increased feeling of fatigue as well as an increase in perceived mental load under unchanged task conditions (Zijlstra, 1993). Earlier studies have demonstrated that control of attention, monitoring of one's actions and response planning in complex tasks are impaired in 
relation to the buildup of mental fatigue (Boksem, Meijman, \& Lorist, 2006; van der Linden, Frese, \& Meijman, 2003). These impairments have two potential consequences: either a decrease of performance or an increase of mental effort expenditure to maintain the same level of performance (Hockey, 1997).

The main goal of the present work was to establish and characterize a possible connection between mental exhaustion and the "baseline" mental activity, as represented by the unconstrained spatial distribution of the ICA-derived RSNs. To this end, we use RS-fMRI and an ICA-based methodology to investigate the effect of sustained performance over the course of several hours on the RSNs of healthy participants.

Our RS-fMRI design incorporated both a free day and a working day for all participants to control for circadian effects (Bartlett et al., 1988). In both days RS-fMRI scans were acquired at the beginning and the end of the day at the same daytimes (+/- 30 minutes), corresponding for the working day to a period before the beginning and after the ending of a number of exhausting activities. To maximize the likelihood of inducing exhaustion in all subjects, we used completely unfamiliar tasks, as these normally demand the highest regulative efforts (Hacker et al., 1978). Specifically, we chose to confront the subjects for the first time with a flight simulator, which is expected to stimulate many concurrent and complex brain activities in a real world scenario.

ICA decompositions were performed on all RS-fMRI scans. The ICA maps from reference scans (free day or beginning of the day) were hierarchically "clustered" within subjects (across days) and between subjects, yielding RSN templates which were unbiased with respect to the factor of interest. Corresponding RSN components were then evaluated in a second-level analysis to detect any "enhancement" or "suppression" effects induced by the sustained mental performance.

In addition to RS-fMRI, we also collected behavioral data at all time points to assess the degree of self-reported exhaustion and verify the possible consequences of exhaustion on cognitive performance and mental effort during a standard N-back task.

We hypothesize that the treatment will lead to an increased level of exhaustion and that the N-back task will be more challenging for the subjects after treatment. While this will not necessarily lead to a decrease in performance, we expect that subjects will need to invest more effort to control the cognitive processes that are required for task performance. As a consequence, we anticipate that, compared to the free day, a day spent with sustained performance would not only increase the (self-reported) levels of exhaustion, but also proportionally influence the RS-fMRI activity in one or more RSNs, causing significant change in the spatial distribution of the corresponding ICA components. Specifically, we expect to find networks that have been associated with maintaining the focus of attention on a task to be affected and that this effect could manifest itself in a loss of coherence in networks that play a role in actively maintaining attention, such as the fronto-parietal executive network (Corbetta and Shulman, 2002). Another possibility would be to find an 
increased coherence in networks known to be negatively correlated to attentionmaintaining networks, such as the default mode network (DMN) (Uddin et al., 2009).

\subsection{Methods}

\subsubsection{Participants}

11 healthy participants (mean age 22.7 years, 4 males) were recruited from the Maastricht University student community. In order to minimize potential artifacts in the lower regions of the frontal cortex, we excluded candidates with orthodontic retainers. Screened participants were invited for the testing session. All participants gave informed consent before the start of the experiment.

\subsubsection{Tasks and Experimental Procedure}

Participants arrived at the lab at the facilities of the Maastricht Brain Imaging Center around 09:30h, (+- 1h). Previously, they were instructed to get their normal amount of sleep, and not to exceed moderate caffeine levels in the morning (maximum of two cups of coffee for habitual users not less than one hour preexperiment). The degree of exhaustion was rated on two 0-150 Visual Analogue Scales (VAS). On one VAS participants were asked to indicate their degree of tiredness, on the other their degree of being rested. Scores of the latter scale were reversed and a mean score of the two scales was calculated.

The participants were then placed in an MRI scanner and the resting state functional and anatomical image data were recorded (see below for MRI parameters). Afterwards, participants performed a version of the n-back task (Gevins and Cutillo, 1993). The task consisted of 15 blocks of one minute each. Participants had to memorize letters appearing on a screen and indicate through a button press response if those letters were identical to the letter 1, 2, or 3 trials back. Each of the three conditions was presented five times, in a quasi-randomized order. Performance was measured as the number of correct button presses within the $2000 \mathrm{~ms}$ response window. Participants received feedback on each trial in order to be able to adjust their effort expenditure. After each one-minute block, participants rated their subjective expenditure of mental effort on the Rating Scale Mental Effort (Zijlstra, 1993). The task and the RSME were programmed in E-Prime (Psychology Software Tools, Inc., US). They were presented using E-Studio on a Windows XP PC connected to an MRI compatible optic system consisting of a projector and mirror goggles. Task and rating input was collected via an MRI compatible optical 2-button Joystick (Current Designs Inc., Philadelphia, USA). Participants trained the handling of the Joystick for a brief period before the experiment by marking values on a VAS 
analogue to the one used in the actual experiment. Also, in order to avoid learning effects or effects of being exposed to the MRI environment for the first time, all subjects performed 15 blocks of the n-back task while being scanned at a separate session around 1-2 weeks before the actual study.

After this session, participants either underwent a $4 \mathrm{~h}$ practice session in the university's helicopter cockpit mock-up or spent the same amount of time with selfchosen, low-effort activities. The practice session was organized in such a way to induce the exhaustion level of a demanding work day.

The helicopter practice session consisted of a short theoretical instruction on helicopter take-off procedures and a practical part of trying to perform a takeoff procedure according to the presented guidelines. After around $2 \mathrm{~h}$, subjects returned to the MRI lab. Here, they again rated their degree of exhaustion on the two VAS and were scanned with the same resting state instructions and scanning parameters as in the morning. This midday session only took place on the working day.

After 2 more helicopter practice hours, participants returned to the MRI lab at around 16:00h. After again indicating their level of exhaustion on the two VAS scales, they were placed back in the scanner. We recorded resting state data with the same parameters and instructions as in the previous sessions. Afterwards, participants performed the same n-back paradigm as in the morning session. All subjects underwent both the free day treatment and the work day treatment in quasirandomized order. In summary, each subject had five RS-fMRI scans, which for the sake of brevity has been labeled according to the day, free day (FD) or working day (WD) and to the time point (T1, T2 and T3). A graphical description of the study protocol is shown in figure 1. 


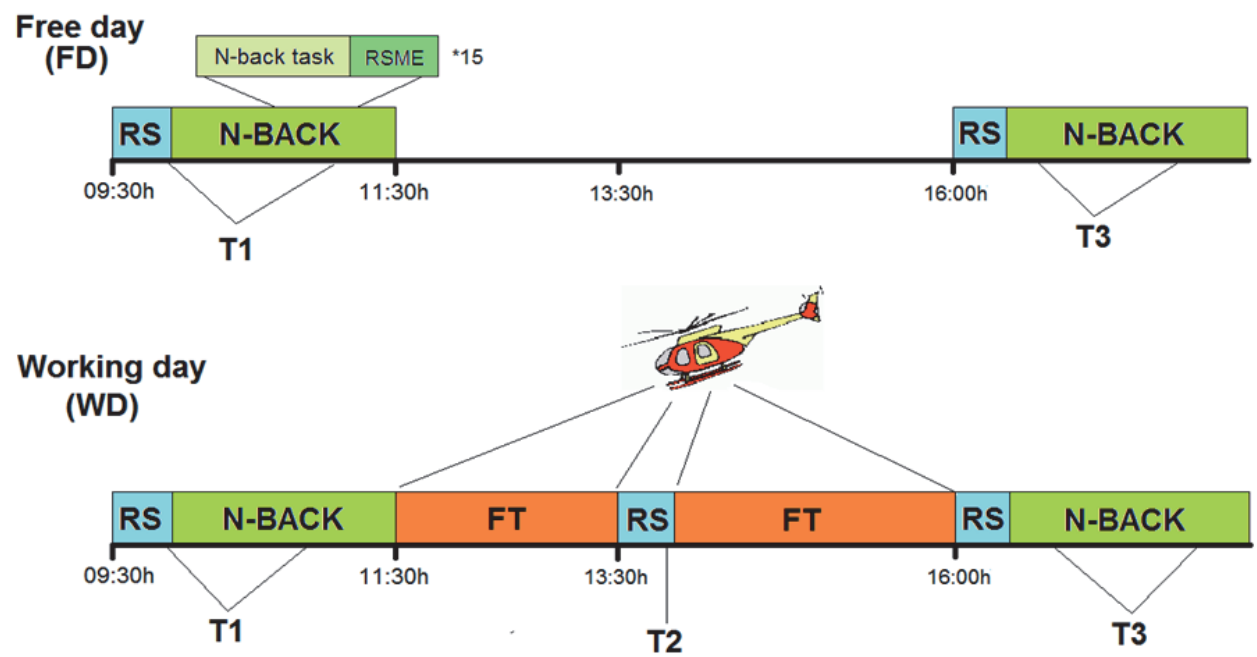

Fig. 1: The research design consisted of two days. On the free day, participants were in the lab only in the morning and in the afternoon. In between, they performed self-chosen, low-effort activities. On the working day, morning and afternoon schedules were kept identical, however, an additional resting state measurement was included in the noon. Time scale is not proportional see methods for timing information.

$\mathrm{RS}=$ resting state; FT = flight task.

\subsubsection{Behavioral data analysis}

Analysis of the behavioral data was conducted in SPSS 18. Behavioral scores of the two exhaustion scales were pooled after reversion of the "rested" scale. Exhaustion and RSME ratings and the number of errors made on the n-back task were $\mathrm{z}$ standardized per participant. For the analysis, we only used the ratings at T1 and T3. One participant reported difficulties with the exhaustion scale and therefore data from this participant were excluded from all analyses. For the analysis of the exhaustion scores, we performed a univariate analysis of variance (ANOVA) with time-of-day (morning: T1 versus evening: T3) and the day activity (work day: WD vs free day: FD) as factors. The dependent variable was the normalized exhaustion score. As the results showed a significant interaction of the time of the day and the day activity, we subsequently contrasted the scores per level of daytime and day activity.

For the effort data, an initial exploratory GLM of effort scores at T1 showed an unexpected effect of day activity at T1. We suspected that this might show learning effects for the n-back tasks. Due to exclusion of 3 participants (see above in this section and section 6.2.5), the order of the working day and the free day were not balanced, as 6 of the remaining 8 participants started with the working day. We thus included the order of test days (first versus second day) as covariate. The subsequent GLMs that we calculated separately for T1 and T3 thus included day activi- 
ties and n-back conditions as factors and the order of test days as a covariate. An influence of our treatment on performance was tested with an identical GLM, only this time using number of errors as the dependent variable.

\subsubsection{MRI and fMRI data acquisition}

The participants were placed in a Siemens Allegra 3T head scanner (Siemens AG, Erlangen, Germany). Respiratory and pulse measurement devices in the form of an expandable breath sensor and an infrared finger clip were fitted on the participants.

The resting state data were recorded with the participant's eyes closed and the instruction to stay awake, but to not engage in any specific mental activity for the time of the scan. Whole brain Echo-Planar Imaging (EPI) was performed using the following parameters: Matrix size 64x64; slice thickness $3 \mathrm{~mm}$; Slice order descending and interleaved; no gap; FOV 192x192mm; TE=30ms; TR=2000ms, 180 repetitions. Slice orientation was tilted in order to minimize susceptibility artifacts in the orbitofrontal regions (Deichmann et al., 2003). Anatomical imaging was carried out with a standard Alzheimer Disease Neuroimaging Initiative (ADNI) complaint T1 weighted sequence, voxel size 1 cubic $\mathrm{mm}$; flip angle $=9 \mathrm{deg} ; \mathrm{TR}=2250 \mathrm{~ms}$; $\mathrm{TE}=$ $2.6 \mathrm{~ms}$.

\subsubsection{MRI and fMRI data preparation}

Standard (f)MRI image data preparation, normalization and pre-processing and statistical analysis and visualization were performed with the BrainVoyager QX software (Brain Innovation B.V., The Netherlands) (Goebel et al., 2006). Functional data preprocessing included the correction for slice scan timing acquisition, the 3D rigid body motion correction and the application of a temporal high-pass filter with cut-off set to 2 cycles per time-course. One participant showed excessive ( $>4 \mathrm{~mm}$ ) movement in at least one of the scanning runs. Additionally, data from one run of another participant were lost due to a file transfer error. Data from both of these participants were excluded from all analyses. Structural and functional data of the remaining 8 subjects were co-registered and spatially normalized to the Talairach standard space. In the course of this procedure, the functional images were resampled to an isometric $3 \mathrm{~mm}$ grid covering the entire Talairach box.

Although ICA separates neural activity from physiological artefacts in most cases, it has been also reported that in some cases a component automatically identified as RSN was the same as the component identified as respiration-related (Birn et al., 2008). Therefore, to account for possible BOLD effects due to cardiac pulsation and respiratory cycle (Birn et al., 2008) physiological noise correction was performed on each functional scan using the RETROICOR technique (Glover et al., 2000). Time-courses for components of heart rate, respiration and respiration volume per time were created from the recorded physiological signals at the fMRI 
sampling rate using Matlab scripts (The Mathworks, United States) available from the AFNI suite (Cox, 1996) and used, together with the motion estimate timecourses available from the previous 3D rigid body motion correction, as predictors in single-study general linear model (GLM) analysis (Friston et al., 1995) of each functional scan. Using the residual time-courses from this GLM allowed us to regress out possible signal fluctuations time-locked with the phase of cardiac and respiratory cycles and residual movement-related signal fluctuations.

\subsubsection{Resting-state fMRI data analysis}

Single-subject and group-level ICA analyses were performed on the pre-processed functional time series and the estimated independent components using two plugin extensions of BrainVoyager QX implementing the fastICA algorithm (Hyvarinen, 1999) and the self-organizing group-level ICA (sog-ICA) algorithm (Esposito et al., 2005; Esposito and Goebel, 2011).

For each subject and each scan, 30 independent components were extracted and scaled to spatial z-scores (i.e. the number of standard deviations of their wholebrain spatial distribution). These values express the relative amount a given voxel is modulated by the activation of the component (McKeown et al., 1998) and hence reflect the amplitude of the correlated fluctuations within the corresponding functional connectivity network (effect size): within the same component, positive and negative ICA values respectively reflect in-phase or out-of-phase fluctuations of a voxel with respect to the network activation. The final number of ICA components is a free parameter, which has previously been either empirically determined or estimated (Calhoun et al., 2009) as the number of principal components retained in the multivariate data. This number typically lies between 20 and 60 depending on the data. In the present work we chose to keep a number of principal components corresponding to one sixth of the number of time points (Greicius et al., 2007) and accounting for more than $99.9 \%$ of the total variance.

The group ICA analysis was conducted in two steps. In the first step, the baseline scans from both days (FD-T1 and WD-T1) were submitted to a "hierarchical" sog-ICA analysis (van de Ven et al., 2009). The purpose of this first step was to determine the most representative RSN template maps to be used in the second step. To avoid day- and treatment bias, the baseline scans of the two days were pooled together in the first level of the hierarchical sog-ICA and the scans corresponding to the exhausted states were not included. The final result of the first step consisted of 30 clusters of 8 components (one per subject) and this was the basis of a randomeffects analysis which was conducted as a 1-factor ANOVA with 1 within-subject factor ("cluster membership") and subjects as random observations. From this ANOVA, we produced 1-sample t-test maps (one for each cluster) and identified at least seven of the most relevant RSN components as reported and illustrated in previous studies (see, e. g., Mantini et al., 2007). For each identified RSN, an RSN 
template mask was obtained by applying a voxel-level threshold of $\mathrm{P}=0.05$ (Bonferroni corrected).

In the second step, all RSN template masks were used to select one component per subject per RSN in each separate scan (Greicius et al., 2004;Greicius et al., 2008). For each RSN, we selected five best-fitting RSN components per subject, one component per condition, and ultimately submitted 32 individual components (4 components per condition: FD/WD_T1/T3, 8 subjects) to a new random effects analysis which was conducted as a 2-factor ANOVA with 2 within subject factors: "day" (F vs D) and "time point ("T1 vs T3"). In order to isolate exhaustion-induced effects, from this 2-way ANOVA, we combined three linear contrasts, respectively accounting for (i) the effect of the treatment across days at T3: [WD-T3 vs FD-T3], (ii) the effect of treatment in the working day: [WD-T3 vs WD-T1] and (iii) the effect of treatment across days and time points [WD-T3 vs FD-T1]. The third contrast was added to exclude any interaction effects implied by the contrast [FD-T3 < FDT1] and not by the contrast [WD-T3>WD-T1]. Combining all these inequalities yields: [3*WD-T3>WD-T1+FD-T1+FD-T3], which is the linear contrast that we have tested to identify the regions with statistically significant effects of "enhancement" or "suppression" of the regional RS-fMRI oscillations. Regional effects were only accepted for compact clusters surviving the joint application of a voxel- and a cluster-level threshold, which were chosen using a non-parametric randomization approach. Namely, an initial uncorrected threshold was applied $(\mathrm{p}=0.01)$ to all voxels and, then, a minimum cluster size was calculated that protected against false positive clusters at 5\% after 500 Monte Carlo simulations (Forman et al., 1995;Goebel et al., 2006). To further correct for the number of studied RSNs, a cluster-corrected level of $5 / \mathrm{N} \%$ was considered, $\mathrm{N}$ being the number of RSNs selected in the first step of the analysis (and therefore the number of actual 2-ANOVA contrasts). For regions passing the full correction criterion or, for an exploratory analysis, at least the cluster-level correction criterion, individual ICA z-scores were extracted for the scans from time points WD-T2 and WD-T3, averaged over all voxels and used for a linear correlation analysis with the degrees of exhaustion, as expressed by the mean VAS score. To compute a pooled statistical significance of these correlations, while correcting for the implicit study effect, we entered the regional ICA scores into a 2-way analysis of covariance (ANCOVA) with one categorical factor (time point), one continuous factor (VAS score) and a time point-by-exhaustion interaction term. Because these analyses were only exploratory, we report their statistical significance level without correction for multiple comparisons. 


\subsection{Results}

\subsubsection{Behavioral results}

In the analysis of the exhaustion scores, the results of the ANOVA showed a significant interaction effect of time-of-day and the day activity $(F(1,31)=10.2, p<0.05)$. Subsequent analysis per level of time-of-day showed that exhaustion was rated higher $(F(1,14)=30.19, p<0.05)$ in the afternoon of the work day (WD-T3) than on the afternoon of the free day (FD-T3). There was no significant difference ( $p>0.05$ ) at the T1 measurement points (see Figure 2).

When testing for differences in RSME ratings at T1, we initially found an unexpected effect of day activity $(F(1,236)=18.98, p<0.05)$. As we suspected a possible learning effect, we repeated the analysis with the order of test days as a covariate. Test day was indeed a significant factor $(F(1,235)=38.46,. p<0.05)$, as was n-back condition $(F(2,235$. $)=107.91, p<0.05)$, while day activity was no longer a significant factor $(F(1,235)=0.93,. p>0.05)$. At T3, order of test days $(F(1,235)=$.10.89 , $p<0.05)$ and n-back condition $(F(2,235)=26.53,. p<0.05)$ were both significant. At this point, however, also day activity was significant $(F(1,235)=5.60,. p<0.05)$ (see Figure 3). The performance of the participants, measured in number of errors, was shown to be influenced at T3 by both the order of test days $(F(1,235)=$.14.78 , $p<0.05)$ and the n-back condition $(F(2,235)=19.81,. p<0.05)$, but not by the day activity $(F(1,235)=2.45,. p>0.05)$.

\section{Exhaustion}

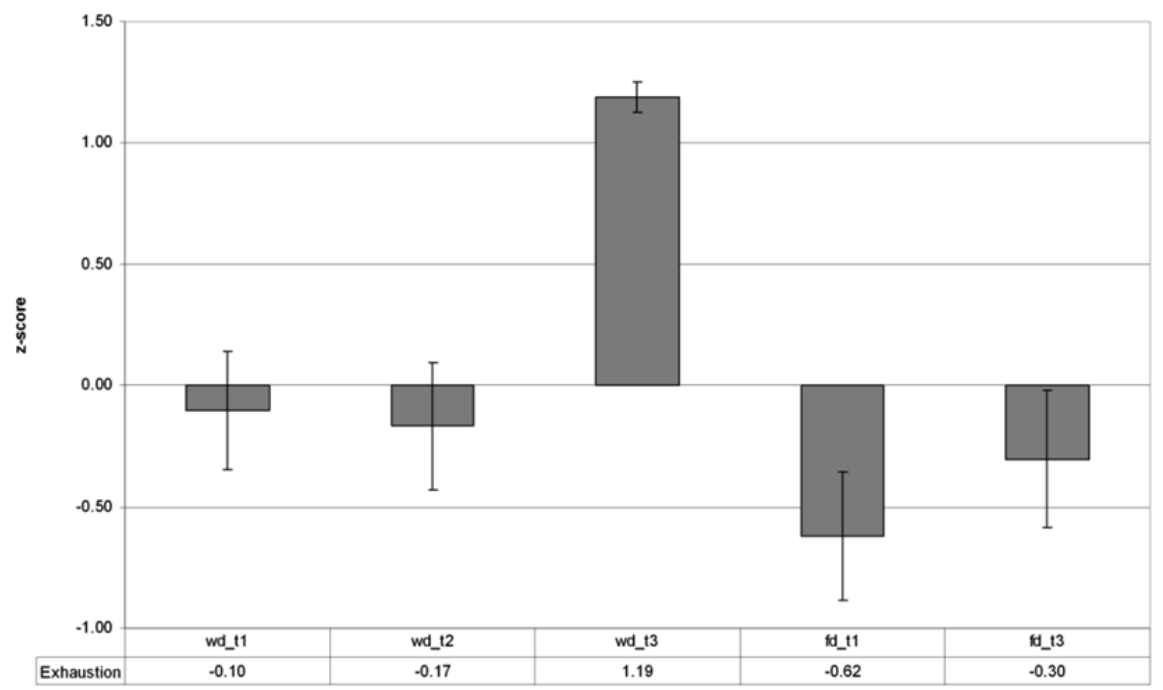

Fig. 2: Exhaustion levels over the course of the work (wd_t1/t3) and the free day (fd_t1/t3) with standard error bars. 


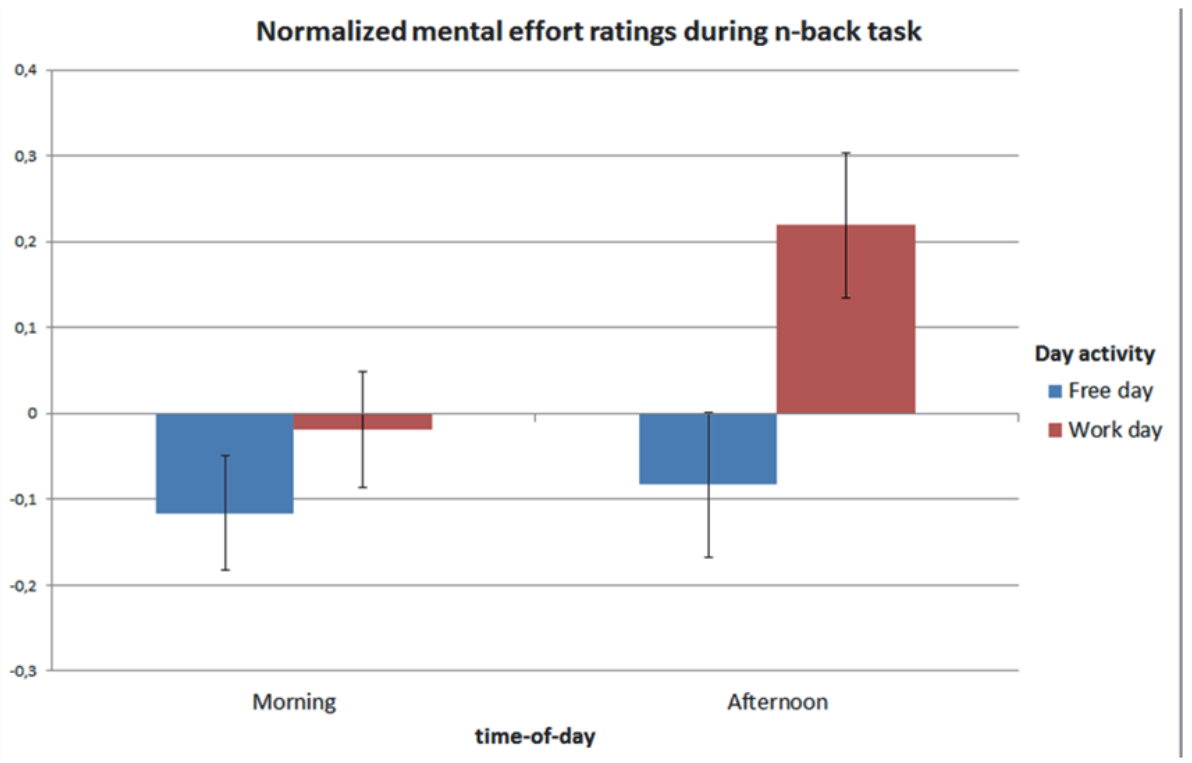

Fig. 3: Effort ratings for all N-back conditions as an effect of treatment (work vs free days): Estimated marginal means per time-of-day and day activity (value of the centered covariate day order $=0$ ). Error bars represent standard errors.

\subsubsection{Imaging results}

In the first step of the analysis including only the morning sessions, at least seven RSN components were identified that were highly similar to those reported in previous ICA-based RS-fMRI studies. These RSN components could be functionally categorized by the Talairach coordinates of the most active sub-regions (for reference, we used, e. g., the table presented by Allen et al. (Allen et al., 2011)) and were accordingly labeled as (two) default-mode networks (an anterior and a posterior default-mode network), a visual network, an auditory network, a sensori-motor network, and two (lateralized) dorso-lateral fronto-parietal networks. These components were selected as "baseline" RSN components for the second step of the analysis (fig. 4). 


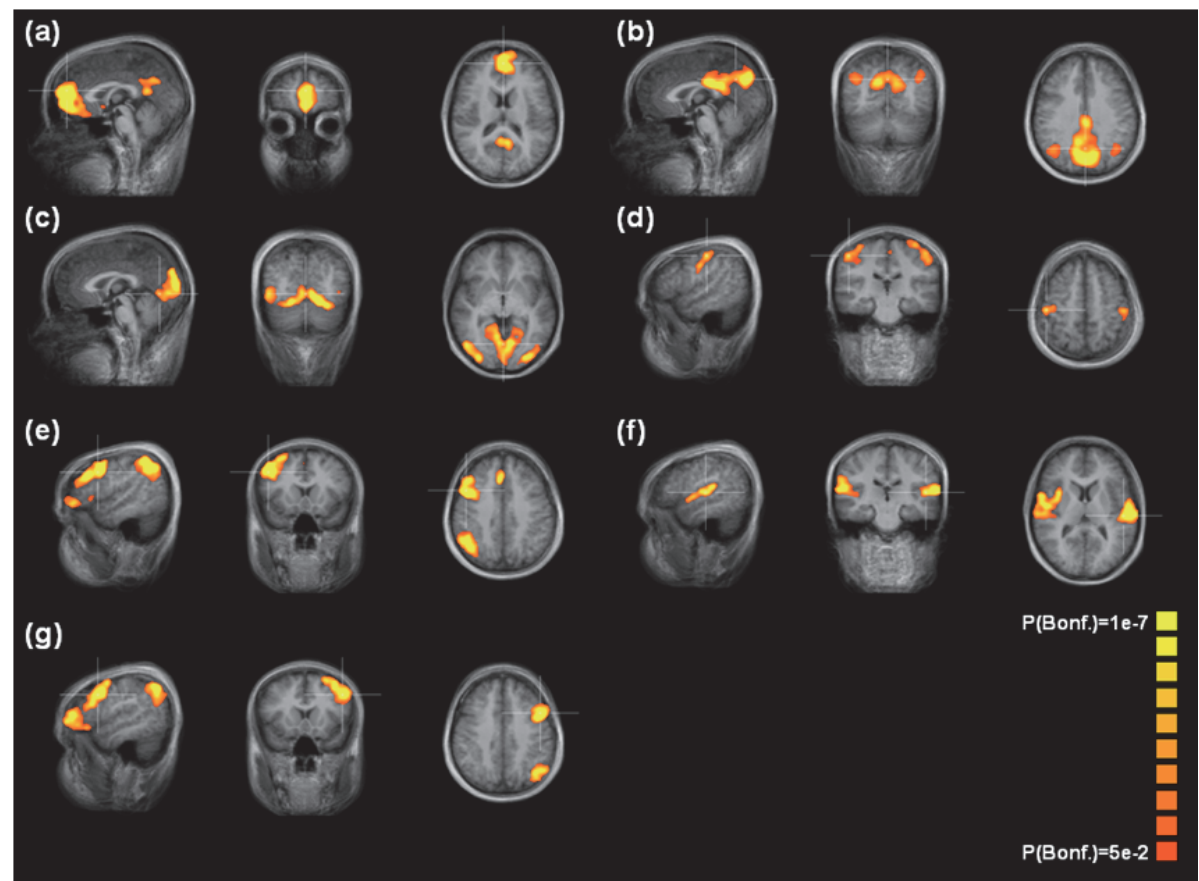

Fig. 4: Group ICA results for the analysis of the pooled baseline resting state scans (WD-T1, FD-T1): (a) anterior default mode network; (b) posterior default mode network; (c) visual network; (d) sensorymotor network; (e) right fronto-parietal network; (f) auditory network; (g) left fronto-parietal network

In the second step of the analysis, that included both free and work sessions, clusters of voxels displaying statistically significant differences in the comparison between the most exhausted condition and the mean of all other conditions were detected within four RSNs. These are reported in table 1 and will be illustrated and discussed below. 
Table 1: Regional effects of exhaustion in resting-state networks (individual clusters).

$\left.{ }^{*}\right) \mathrm{P}<0.05$ cluster-level corrected.

$(* *) \mathrm{P}<0.05$ cluster- and network-level corrected.

\begin{tabular}{|c|c|c|c|c|c|}
\hline $\begin{array}{l}\text { Resting-state } \\
\text { Network }\end{array}$ & Anatomical Region & $\begin{array}{l}\text { Center of Mass } \\
\mathrm{x}, \mathrm{y}, \mathrm{z} \text { (Talairach) }\end{array}$ & $\begin{array}{l}\text { T-stat } \\
{[\text { Avg, Max }]}\end{array}$ & $\begin{array}{l}\text { Extension } \\
{\left[\mathrm{mm}^{3}\right]}\end{array}$ & $\begin{array}{l}\text { Correlation with } \\
\text { Exhaustion } \\
\text { [F-stat, } p \text {-value] }\end{array}$ \\
\hline Early Visual & Left Lingual Gyrus & $-16,-62,0$ & $\begin{array}{l}+4.16 \\
+6.77\end{array}$ & $1796(* *)$ & $6.7,0.0237$ \\
\hline $\begin{array}{l}\text { Anterior } \\
\text { Default-mode }\end{array}$ & Medial Frontal Gyrus & $-1,+46,+33$ & $\begin{array}{l}+4.06 \\
+6.24\end{array}$ & $380(*)$ & $6.04,0.03$ \\
\hline $\begin{array}{l}\text { Left Fronto- } \\
\text { Parietal }\end{array}$ & Left Middle Frontal Gyrus & $-34,+7,+48$ & $\begin{array}{l}-4.59 \\
-9.82\end{array}$ & $2590(* *)$ & $0.01,0.93$ \\
\hline $\begin{array}{l}\text { Right Fronto- } \\
\text { Parietal }\end{array}$ & Right Angular Gyrus & $+46,-61,+36$ & $\begin{array}{l}-3.50 \\
-7.369\end{array}$ & $887(* *)$ & $0.57,0.46$ \\
\hline $\begin{array}{l}\text { Right Fronto- } \\
\text { Parietal }\end{array}$ & Right Middle Frontal Gyrus & $+33,+7,+35$ & $\begin{array}{l}-4.24 \\
-6.568\end{array}$ & $911(* *)$ & $2.52,0.13$ \\
\hline
\end{tabular}

We found statistically significant regional effects of exhaustion in the early visual processing network (fig. 5), in the anterior default mode network (fig. 6) and in both the right and the left dorso-lateral fronto-parietal networks (fig.7 ), corresponding to the two executive attentional networks.

In the visual processing network (fig. 5), a compact cluster located in left lingual gyrus showed a statistically significant regional effect, with rs-fMRI signals enhanced in the most exhausted condition ( $\mathrm{p}<0.01$, clus. size $>702 \mathrm{~mm}^{3}$, cluster- and network-level corrected). Extracting the average ICA scores in this region from each individual revealed a statistically significant positive correlation with the selfreported exhaustion scores $(\mathrm{p}<0.05)$ but no significant scan-by-exhaustion interaction $(\mathrm{p}>0.05)$. 


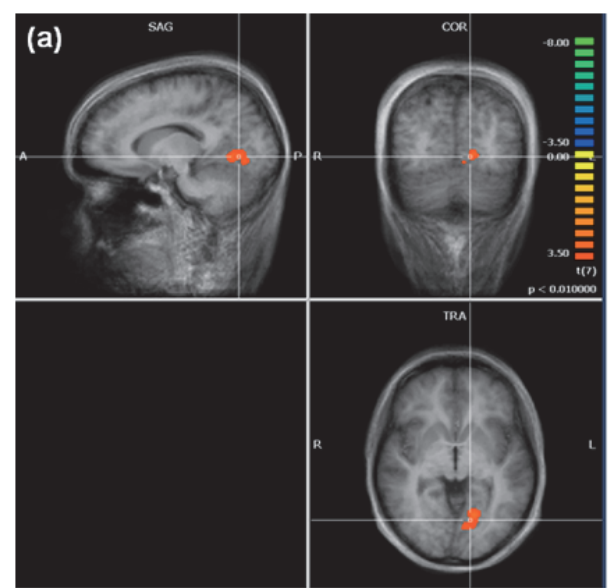

(b)

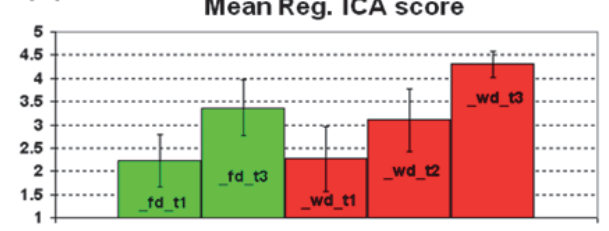

(c)

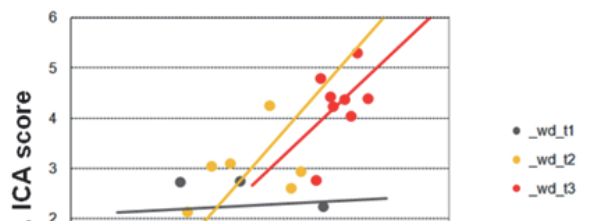

- _wd_12

Fig. 5: Early visual processing network. (a) Statistical map of the differential effects (WD_T3 > WD_T1 + FD_T1 + FD_T3). (b) Bar graph of the regional ICA scores (with standard error bars) in all conditions. (c) Correlation graph of the regional ICA scores against the normalized degree of exhaustion for the separate working day sessions (with fit lines indicating the directions of the correlations).

We found no significant effects in the anterior and posterior default mode networks. At an uncorrected level of significance, in the anterior default mode network, a compact cluster located in medial frontal gyrus showed an effect similar to that observed in the early visual processing network $(\mathrm{p}<0.01$, clus. size $>378 \mathrm{~mm} 3$, cluster-level corrected) with a positive correlation between the regional ICA scores and the exhaustion scores $(\mathrm{p}<0.05)$ (fig. 6) and no significant scan-by-exhaustion interaction $(\mathrm{p}>0.05)$. 


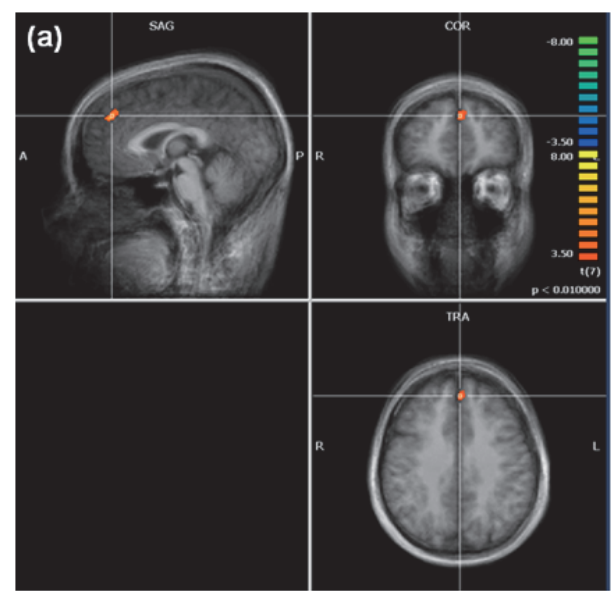

(b)

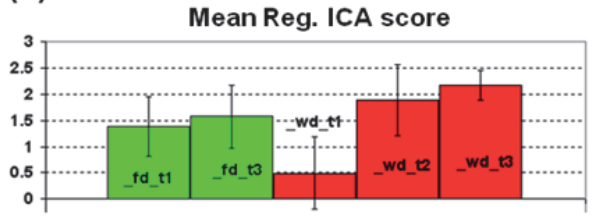

(c)

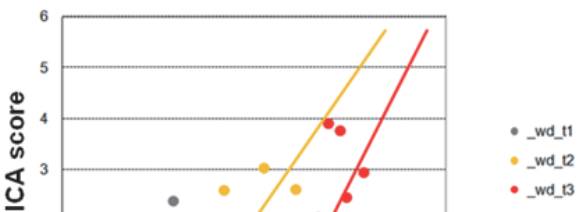

_wd_12

- _wd_13

Fig. 6: Anterior default mode network. (a) Statistical map of the differential effects (WD_T3 > WD_T1 + FD_T1 + FD_T3). (b) Bar graph of the regional ICA scores (with standard error bars) in all conditions. (c) Correlation graph of the regional ICA scores against the normalized degree of exhaustion for the separate working day sessions (with fit lines indicating the directions of the correlations).

Finally, both left and right fronto-parietal executive attentional networks exhibited reduced functional connectivity in the most exhausted state compared to all other states. More specifically, in the left fronto-parietal executive attention network, a compact cluster located in left middle frontal gyrus showed an effect opposite to that observed in the anterior default mode and the visual processing networks ( $p<0.01$, clus. size $>675 \mathrm{~mm}^{3}$, cluster- and network-level corrected). In the right fronto-parietal network, the suppression of RS-fMRI signals was distributed across the anterior and posterior nodes. In fact, two compact clusters $(\mathrm{p}<0.01$, cluster size $>540 \mathrm{~mm}^{3}$, cluster- and network-level corrected) located anteriorly in right middle frontal gyrus and posteriorly in the right angular gyrus exhibited a similar significant suppression. However, for none of the regions, the correlation with behavioral scores or the scan-by-exhaustion interaction reached statistical significance $(\mathrm{p}>0.05)$. 


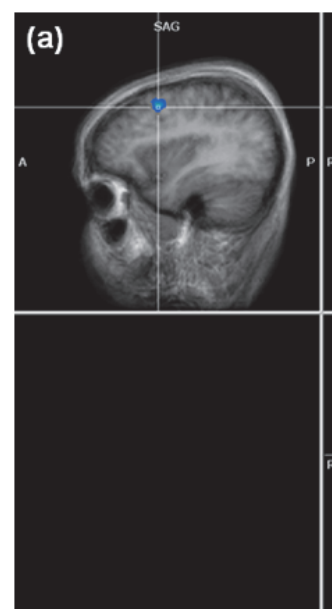

(b)

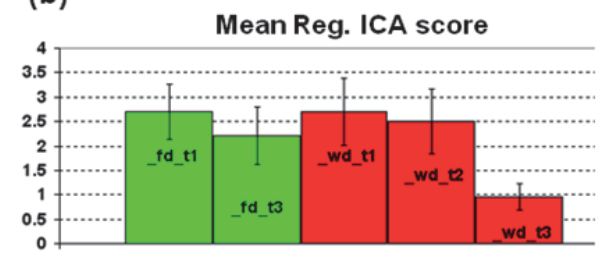

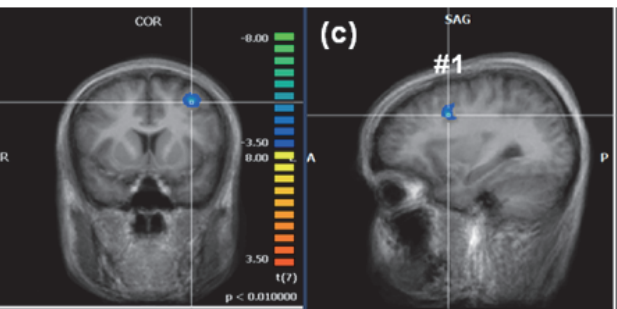
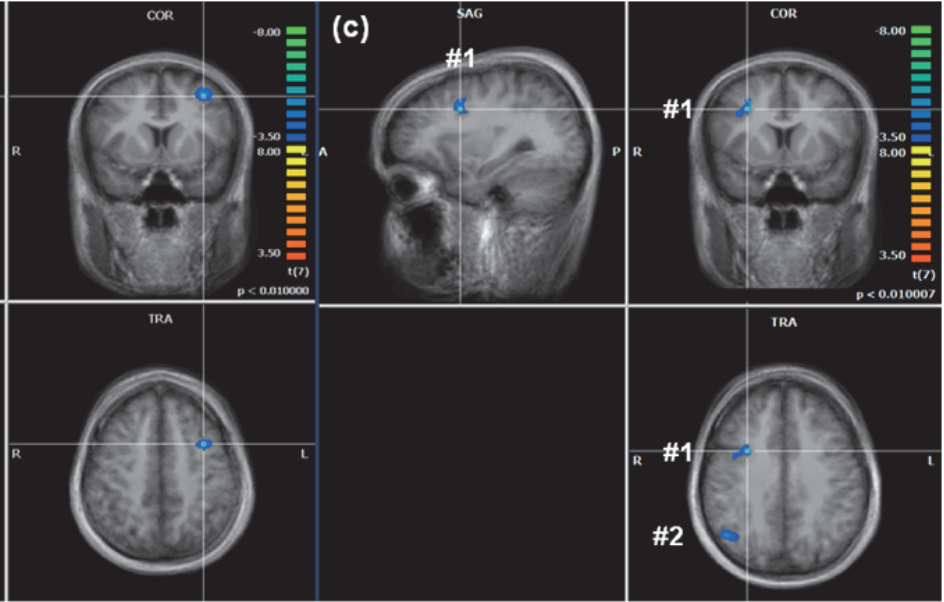

(d)

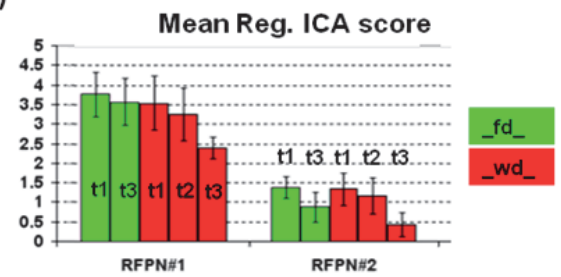

Fig. 7: Left $(a, b)$ and right $(c, d)$ fronto-parietal networks. $(a, c)$ Statistical maps of the differential effects (WD_T3 > WD_T1 + FD_T1 + FD_T3). (b,d) Bar graphs of the regional ICA scores (with standard error bars) in all conditions. 


\subsection{Discussion}

In line with previous studies, the combination of an RS-fMRI experimental design with ICA-based methodology has gathered a robust task-independent fractionation of the spontaneous brain activity into multiple domain-specific spatial patterns. In this study, we used spatially distributed ICA patterns of RS-fMRI activities, first, to establish a task-independent link between system neurophysiology and behavioral psycho-physiology, and, second, to address the possible modulation of the functional connectivity in the prominent RSNs by an intensive psychological treatment without constraining the brain regions of interest on the basis of prior anatomical or functional knowledge. More specifically, we explored the distributed topological changes of the main RSNs in healthy volunteers who spent an entire full day in completing challenging and demanding tasks, which caused abnormal levels of mental exhaustion without impairing the normal performances in a standard cognitive test. By using a rigorous multi-level ICA-based approach, we demonstrated domain-specific spatially selective effects which were in some cases correlated with the increased levels of mental exhaustion reported by the studied subjects.

\subsubsection{Subjective reports of state and effort investment}

The behavioral results were in line with our hypotheses. Namely, the sustained performance in the working day induced an increased self-reported level of exhaustion compared to the free day. Although, theoretically, using a self-report instrument for exhaustion could have lead the participants to deduce our key interest and give desired ratings, this seems unlikely in this particular case. In fact, exhaustion was just one of several self-reports that was assessed, with other variables such as well-being and emotional state being collected for an undergraduate project that was linked to this study.

In the working day, there was no significant difference between exhaustion levels at $\mathrm{T} 1$ and $\mathrm{T} 2$. We speculate that the short training periods were not enough to actually exhaust the participants strongly already at T2. This reflects our rationale to design a paradigm featuring longer task duration. In fact, a short period of activity in a stimulating environment such as a flight simulator can be assumed to actually increase arousal and thereby decrease experienced exhaustion. Also, it is possible that at $\mathrm{T} 1$, participants still experienced effects of sleep inertia and low body temperature, as their bodies just started to wake up (see, e. g., Valdez et al., 2008). Nonetheless, when correlating brain and behavior, we pooled data from both T2 and T3 as we did not want to exclude the possibility that functional brain networks could predict potential sub-threshold exhaustion effects already at T2. 
Next to an increase in exhaustion, we also found a significant increase of mental effort expenditure to maintain the same performances (i. e. same error rates) in the $\mathrm{N}$-back task at the end of the working day compared to the free day at identical day times. This means that participants were able to sustain their performance level, yet they had to invest a significantly higher amount of mental effort. This finding is consistent with the framework of Hockey (Hockey, 1997) and similar findings have been demonstrated earlier (see, e. g., van der Linden et al., 2003).

\subsubsection{Changes in functional connectivity related to exhaustion}

Another important objective of the present work was to establish whether a significantly higher than normal level of mental exhaustion would be also reflected in different amounts of functional connectivity in certain brain networks at rest, i.e. prior to (and independent of) any specific task or performance, and to what extent the amount and synchrony of RS-fMRI signals from certain regions within certain RSNs would be predictive of the subjective measure of fatigue. Therefore we decomposed whole-brain RS-fMRI into spatial independent components and compared the distributions of the most typical RSN components on a voxel by voxel basis to detect possible regional differences between an exhausted and a control resting brain state. This approach has a number of advantages over the use of seed voxel-based approaches (Esposito and Goebel, 2011) and provides a voxel-wise measure of functional connectivity that reflects the correlation between the activity of each voxel and the rest of the network being tested.

First of all, we found a remarkable effect of the work treatment on the early visual processing network, where the RS-fMRI fluctuations were significantly higher in the exhausted state and the regional scores were positively correlated with the degree of exhaustion. This RSN has been previously found to be principally associated with the electro-encephalographic (EEG) "alpha" rhythm (Mantini et al., 2007), which is typically more prominent in exhausted states, such as, e. g., after sustained visual attention (Boksem et al., 2006) or driving tasks (Kamei et al., 1998; Schrauf et al., 2011), but also proportionally more attenuated in relaxed states, such as, e. g., after a daytime nap (Tamaki et al., 1999).

Most relevant to our own findings, a recent study by Lim et al. (Lim, CatherineQuevenco, \& Kwok, 2013) has demonstrated a correlation of alpha band power as a predictor of successful recovery from a sustained mental task during a short break. Participants demonstrating a lower level of alpha power in a resting period between two blocks of task performance showed both a stronger decrease in frontally focused theta power from pre- to post- break and a higher increase in performance after the break. Upper alpha band power during the break was found to be the moderator between theta band power reduction and performance differences across the break. 
Taking these evidences together, and considering that it is generally believed that the presence of alpha oscillations signifies "idling" or inhibition of cortical processing (Pfurtscheller et al., 1996; Worden et al., 2000), the observed regional increase of the RS-fMRI signal fluctuations in the visual network induced by exhaustion clearly points to increased inhibition of the neural activity in the early visual system implied by exhaustion.

Albeit only descriptively, we also report a weak positive effect of exhaustion in the anterior DMN, characterized by the regional increase of RS-fMRI signal fluctuations in the medial frontal gyrus. In this spot, the correlation between the mean regional ICA scores and the self-rated degree of exhaustion was also positive and statistically significant in the exhausted states. A similar effect of increased resting state frontal connectivity in the anterior DMN has been recently observed in patients with mild traumatic brain injury (Zhou et al., 2012). In contrast to this study, however, no significant effects were found in the posterior DMN.

Different from what has been observed in the early visual processing and the anterior default mode networks, both the right and the left dorso-lateral frontoparietal networks were found to be suppressed in the exhausted, compared to the relaxed states, with clusters of significantly negative differences in frontal and parietal lateralized regions.

The suppression of the fronto-parietal networks could be a sign that brain fatigue is deteriorating the subjects' executive abilities. In fact, the suppressed areas in these networks have long been established as crucial in the effortful maintenance of sustained attention (Lewin et al., 1996; Cabeza and Nyberg, 2000; Corbetta and Shulman, 2002; Kim et al., 2006; Lim et al., 2010; Demeter et al., 2011), therefore the functional connectivity of these networks could be impaired after long periods of sustained activation with associated heavy mental workload. In our experimental and analytical framework, this possible RSN impairment was detected before any visible performance degradation in the subsequent N-back tasks.

\subsubsection{Evidence for a bidirectional relation between RSNs and performance}

The suppression of fronto-parietal networks observed with RS-fMRI in a significantly exhausted state could be linked to recent arterial spin labeling (ASL) perfusion based fMRI (P-fMRI) results (Lim et al., 2010; Demeter et al., 2011).

Lim et al. (Lim et al., 2010) showed that sustained periods of taxing cognitive workload represented by 20 -min executions of a psychomotor vigilance test (PVT), besides measurable performance declines, also cause significant deactivations (as reflected by regional cerebral flow ( $\mathrm{rCBF}$ ) reductions) in a right fronto-parietal network during a post-task resting interval, compared to a pre-task resting baseline. Remarkably, it is this $\mathrm{rCBF}$ decrease between post- and pre-task baselines, and not the rCBF increase during the task, that turns out to predict the PVT perfor- 
mance decline, suggesting the presence of persistent effects of cognitive fatigue in the right fronto-parietal network after a period of heavy mental work, as well as the critical role of this executive attention network in mediating the (temporary) impairment of cognitive abilities.

Even if RS-fMRI suppression occurred in both dorsal fronto-parietal networks, this effect appeared more widespread and distributed over distant regions in the right lateralized network, where both frontal and parietal nodes were suppressed, than in the left dorsal fronto-parietal network, where only one anterior region in the left middle frontal gyrus was suppressed. In other words, the suppression appears to be a more generalized phenomenon in the right fronto-parietal network, even if the correlation with the self-rated degree of exhaustion does not reach statistical significance in any of the single spots of regional differences detected. Contrariwise, the suppression is stronger in the left fronto-parietal network, suggesting a potentially worse damage in the anterior portion of this network.

In line with the idea that more sustained activity during the tasks causes more deterioration to the executive attention network after the tasks, the more widespread involvement of the right fronto-parietal network could be linked to the right lateralization reported (in both human and monkey studies) when these areas are engaged in tasks requiring attention, vigilance and continuous performance (Corbetta et al., 1993; Colby and Duhamel, 1996; Nobre et al., 1997; Coull et al., 1998; Cabeza and Nyberg, 2000; Fan et al., 2005; Ogg et al., 2008). In the working day, i. e. during intensive helicopter flying practice in the simulator, subjects were confronted with a task that required continuous attention and careful control of the cockpit interfaces to successfully operate the aircraft. Hence, the task requirements in our study can be assumed to strongly engage the right fronto-parietal network, even if we cannot be as specific as to predict which aspect of the task in particular is responsible for the reported increase of exhaustion and the suppression of the right fronto-parietal network. Contrariwise, the higher deterioration observed in the left middle frontal gyrus (compared to its contra-lateral counterpart) could be attributed to the fact that in healthy (non-depressed) subjects cognitive performance inducing stress, causes abnormally higher activations in left compared to the right middle frontal gyrus during the same working memory task (Koric et al., 2011). Thereby, it is plausible that this kind of stress could have contributed to the more regionally specific deterioration of the left fronto-parietal network.

Other groups have previously addressed possible alterations in RSN functional connectivity and their endogenous dynamic features that can be related to cognitively effortful tasks. For instance, using a similar "rest-task-rest" design, Barnes et al. (Barnes et al., 2009) found that brain endogenous dynamics tend to recover some of their pre-task dynamic features relatively slowly, suggesting that largescale neurocognitive systems can take a considerable period of time to return to a stable baseline state after demanding task. In line with this theory, Gordon et al. (Gordon et al., 2012) demonstrated that after a working memory task, the immediately following resting state functional connectivity remained persistently altered 
compared to the baseline resting state, in a fashion similar to how this was altered during the task performance. Particularly, when examining the task positive network (TPN) and the DMN, a suppression of the within-network functional connectivity in the TPN (as well as a modest non-significant enhancement of the DMN) was observed in this study which seems highly relevant to the interpretation of the present findings. In fact, following Gordon et al. (Gordon et al., 2012), the observed reduction of the functional connectivity in the fronto-parietal networks may well reflect a cognitive after-effect, such that the brain continues to ruminate with the flight simulation after the task is ceased, or, more likely, the persistence of subjective aspects from the recent effortful cognitive experience.

Some studies examining pre vs. post task resting state functional connectivity have also suggested or demonstrated the existence of learning effects in multiple domains, albeit not all in the same RSNs and regions, and not all in the same direction (i. e. manifesting as positive or negative changes). Duff et al. (Duff et al., 2008) have shown negative changes in the sensorimotor network following a simple finger tapping exercise. Using motor learning paradigms, Albert et al. (Albert et al., 2009) have reported an increased functional connectivity in the left frontotemporal network, whereas Vahdat et al. (Vahdat et al., 2011) found that the only regions showing functional connectivity changes purely associated with motor learning were located in the cerebellar cortex and in the superior parietal lobule and all exhibited negative correlations with learning. Using a visual learning paradigm, Lewis et al. (Lewis et al., 2011) were in line with Albert et al. (Albert et al., 2009) in that within-network learning effects would mainly consist of increased resting state functional connectivity that were related to highly specific synaptic mechanisms stimulated by the learning task. Finally, both Waites et al. (Waites et al., 2005) and Grigg and Grady (Grigg and Grady, 2010) used different language tasks to show a positive after-effect of the cognitive activity in which positive changes in resting connectivity were prevalent in high order areas. The common denominator of all these evidences is that learning effects generally cause positive changes in the resting state functional connectivity of high order brain regions and networks, whereas low order domains, such as the sensorimotor or visual domains, might require highly specific learning tasks to show similar learning-related effects prevailing over perceptual or fatigue-related effects. Along this line, considering that our stimulation was not specific to any of the studied low order systems (but rather engaged all brain systems in highly and purposely effortful performances), and that we observed a reduction of the resting state functional connectivity in both the left and the right FPN, we suggest that the learning factor was not as prominent in our experimental design as the exhaustion factor. 


\subsubsection{Limitations, outlook and conclusion}

This study has two important limitations. First, given the low number of subjects, the reported analyses are to be considered only exploratory and, therefore, all results are at present useful only for elaborating initial working hypotheses in the design of future studies. In fact, although we collected five scans per subject, ending up with forty individual scans in total, our approach relies on individual scan ICA components which are known to be noisier and less reproducible than aggregate scan ICA components (Zuo et al., 2010) and, thus, potentially less useful for a small group ICA. Second, it should be considered that the N-back task, while very common and popular in cognitive experiments, could be not appropriate in relation to possible learning effects induced by the practice with the flight simulator. In our experiments, it was not possible (due to technical problems) to record objective indicators regarding the performance at the simulation and therefore the possibility that the extensive practice with the simulator might also have caused that subjects gain knowledge of new skills, cannot be excluded. On the other hand, several studies have shown that apparently less efficient mental processes (resulting, e. g., in increased reaction time on task) can actually correspond to improved performances due to practice (see, e. g., Ackerman and Kanfer, 2009).

In conclusion, this study demonstrates the potential utility of RS-fMRI, in combination with ICA-based distributed modeling of the spontaneous BOLD activity synchronization, for revealing and characterizing the neural networks that present some immediately visible effects of mental exhaustion after sustained and highly demanding training and practice. In fact, our results demonstrate that some of the ICA components corresponding to the most important and reported RSNs exhibit regional changes in their spatial distribution which can be associated with persistent effects of exhaustion, as measurable in the immediate aftermath of a period of heavy cognitive workload. Therefore, characterizing the resting state functional connectivity of these RSNs with ICA may provide novel markers of cognitive fatigue and mental exhaustion useful for identifying neural "risk factors" for accidents and errors due to prolonged task performance. For instance, considering the specific paradigm used here, this framework could be potentially useful to identify risk factors in pilots in relation to, e. g., the maximum number of flight hours allowed. Moreover, a possible clinical implementation of this paradigm could help neurologists to address the effects of cognitive fatigue in neurological diseases (Langdon, 2011). 


\section{References}

Ackerman, P.L., and Kanfer, R. (2009). Test length and cognitive fatigue: an empirical examination of effects on performance and test-taker reactions. J Exp Psychol Appl 15, 163-181.

Albert, N.B., Robertson, E.M., and Miall, R.C. (2009). The resting human brain and motor learning. Curr Biol 19, 1023-1027.

Allen, E.A., Erhardt, E.B., Damaraju, E., Gruner, W., Segall, J.M., Silva, R.F., Havlicek, M., Rachakonda, S., Fries, J., Kalyanam, R., Michael, A.M., Caprihan, A., Turner, J.A., Eichele, T., Adelsheim, S., Bryan, A.D., Bustillo, J., Clark, V.P., Feldstein Ewing, S.W., Filbey, F., Ford, C.C., Hutchison, K., Jung, R.E., Kiehl, K.A., Kodituwakku, P., Komesu, Y.M., Mayer, A.R., Pearlson, G.D., Phillips, J.P., Sadek, J.R., Stevens, M., Teuscher, U., Thoma, R.J., and Calhoun, V.D. (2011). A baseline for the multivariate comparison of resting-state networks. Front Syst Neurosci 5, 2.

Barnes, A., Bullmore, E.T., and Suckling, J. (2009). Endogenous human brain dynamics recover slowly following cognitive effort. PLoS One 4, e6626.

Bartlett, E.J., Brodie, J.D., Wolf, A.P., Christman, D.R., Laska, E., and Meissner, M. (1988). Reproducibility of cerebral glucose metabolic measurements in resting human subjects. J Cereb Blood Flow Metab 8, 502-512.

Birn, R.M., Murphy, K., and Bandettini, P.A. (2008). The effect of respiration variations on independent component analysis results of resting state functional connectivity. Hum Brain Mapp 29, 740-750.

Biswal, B., Yetkin, F.Z., Haughton, V.M., and Hyde, J.S. (1995). Functional connectivity in the motor cortex of resting human brain using echo-planar MRI. Magn Reson Med 34, 537-541.

Boksem, M.a.S., Meijman, T.F., and Lorist, M.M. (2006). Mental fatigue, motivation and action monitoring. Biological Psychology 72, 123-132.

Cabeza, R., and Nyberg, L. (2000). Imaging cognition II: An empirical review of 275 PET and fMRI studies. J Cogn Neurosci 12, 1-47.

Calhoun, V.D., Liu, J., and Adali, T. (2009). A review of group ICA for fMRI data and ICA for joint inference of imaging, genetic, and ERP data. Neuroimage 45, S163-172.

Colby, C.L., and Duhamel, J.R. (1996). Spatial representations for action in parietal cortex. Brain Res Cogn Brain Res 5, 105-115.

Corbetta, M., Miezin, F.M., Shulman, G.L., and Petersen, S.E. (1993). A PET study of visuospatial attention. J Neurosci 13, 1202-1226.

Corbetta, M., and Shulman, G.L. (2002). Control of goal-directed and stimulus-driven attention in the brain. Nat Rev Neurosci 3, 201-215.

Coull, J.T., Frackowiak, R.S., and Frith, C.D. (1998). Monitoring for target objects: activation of right frontal and parietal cortices with increasing time on task. Neuropsychologia 36, 1325-1334.

Cox, R.W. (1996). AFNI: software for analysis and visualization of functional magnetic resonance neuroimages. Comput Biomed Res 29, 162-173.

Damoiseaux, J.S., Rombouts, S.A., Barkhof, F., Scheltens, P., Stam, C.J., Smith, S.M., and Beckmann, C.F. (2006). Consistent resting-state networks across healthy subjects. Proc Natl Acad Sci U S A 103, 13848-13853.

De Luca, M., Beckmann, C.F., De Stefano, N., Matthews, P.M., and Smith, S.M. (2006). fMRI resting state networks define distinct modes of long-distance interactions in the human brain. Neuroimage 29 , 1359-1367.

Deichmann, R., Gottfried, J.A., Hutton, C., and Turner, R. (2003). Optimized EPI for fMRI studies of the orbitofrontal cortex. Neurolmage 19, 430-441.

Demeter, E., Hernandez-Garcia, L., Sarter, M., and Lustig, C. (2011). Challenges to attention: a continuous arterial spin labeling (ASL) study of the effects of distraction on sustained attention. Neuroimage 54, 1518-1529.

Duff, E.P., Johnston, L.A., Xiong, J., Fox, P.T., Mareels, I., and Egan, G.F. (2008). The power of spectral density analysis for mapping endogenous BOLD signal fluctuations. Hum Brain Mapp 29, 778-790.

Esposito, F., and Goebel, R. (2011). Extracting functional networks with spatial independent component analysis: the role of dimensionality, reliability and aggregation scheme. Curr Opin Neurol 24, 378385. 
Esposito, F., Pignataro, G., Di Renzo, G., Spinali, A., Paccone, A., Tedeschi, G., and Annunziato, L. (2010). Alcohol increases spontaneous BOLD signal fluctuations in the visual network. Neuroimage 53, 534543.

Esposito, F., Scarabino, T., Hyvarinen, A., Himberg, J., Formisano, E., Comani, S., Tedeschi, G., Goebel, R., Seifritz, E., and Di Salle, F. (2005). Independent component analysis of fMRI group studies by selforganizing clustering. Neuroimage 25, 193-205.

Fan, J., Mccandliss, B.D., Fossella, J., Flombaum, J.I., and Posner, M.I. (2005). The activation of attentional networks. Neuroimage 26, 471-479.

Forman, S.D., Cohen, J.D., Fitzgerald, M., Eddy, W.F., Mintun, M.A., and Noll, D.C. (1995). Improved assessment of significant activation in functional magnetic resonance imaging (fMRI): use of a cluster-size threshold. Magn Reson Med 33, 636-647.

Friston, K.J., Holmes, A.P., Poline, J.B., Grasby, P.J., Williams, S.C., Frackowiak, R.S., and Turner, R. (1995). Analysis of fMRI time-series revisited. Neuroimage 2, 45-53.

Gevins, A., and Cutillo, B. (1993). Spatiotemporal dynamics of component processes in human working memory. Electroencephalography and clinical Neurophysiology 87, 128-143.

Glover, G.H., Li, T.Q., and Ress, D. (2000). Image-based method for retrospective correction of physiological motion effects in fMRI: RETROICOR. Magn Reson Med 44, 162-167.

Goebel, R., Esposito, F., and Formisano, E. (2006). Analysis of functional image analysis contest (FIAC) data with brainvoyager QX: From single-subject to cortically aligned group general linear model analysis and self-organizing group independent component analysis. Hum Brain Mapp 27, 392-401.

Gordon, E.M., Breeden, A.L., Bean, S.E., and Vaidya, C.J. (2012). Working memory-related changes in functional connectivity persist beyond task disengagement. Human brain mapping.

Greicius, M.D., Flores, B.H., Menon, V., Glover, G.H., Solvason, H.B., Kenna, H., Reiss, A.L., and Schatzberg, A.F. (2007). Resting-state functional connectivity in major depression: abnormally increased contributions from subgenual cingulate cortex and thalamus. Biol Psychiatry 62, 429-437.

Greicius, M.D., Kiviniemi, V., Tervonen, O., Vainionpaa, V., Alahuhta, S., Reiss, A.L., and Menon, V. (2008). Persistent default-mode network connectivity during light sedation. Hum Brain Mapp 29, 839-847.

Greicius, M.D., Srivastava, G., Reiss, A.L., and Menon, V. (2004). Default-mode network activity distinguishes Alzheimer's disease from healthy aging: evidence from functional MRI. Proc Natl Acad Sci U S A 101, 4637-4642.

Grigg, O., and Grady, C.L. (2010). Task-related effects on the temporal and spatial dynamics of restingstate functional connectivity in the default network. PLoS One 5, e13311.

Gusnard, D.A., and Raichle, M.E. (2001). Searching for a baseline: functional imaging and the resting human brain. Nat Rev Neurosci 2, 685-694.

Hacker, W., Plath, H.E., Richter, P., and Zimmer, K. (1978). Internal representation of task structure and mental load of work: approaches and methods of assessment. Ergonomics 21, 187-194.

Hockey, G.R.J. (1997). Compensatory control in the regulation of human performance under stress and high workload: A cognitive-energetical framework. Biological Psychology 45, 73-93.

Hyvarinen, A. (1999). Fast and robust fixed-point algorithms for independent component analysis. IEEE Trans Neural Netw 10, 626-634.

Hyvarinen, A., Karhunen, J., and Oja, E. (2001). Independent Component Analysis. New York: John Wiley \& Sons, Inc.

Kamei, T., Kumano, H., Iwata, K., and Yasushi, M. (1998). Influences of long- and short-distance driving on alpha waves and natural killer cell activity. Percept Mot Skills 87, 1419-1423.

Khalili-Mahani, N., Zoethout, R.M., Beckmann, C.F., Baerends, E., De Kam, M.L., Soeter, R.P., Dahan, A., Van Buchem, M.A., Van Gerven, J.M., and Rombouts, S.A. (2011). Effects of morphine and alcohol on functional brain connectivity during "resting state":A placebo-controlled crossover study in healthy young men. Hum Brain Mapp.

Kim, J., Whyte, J., Wang, J., Rao, H., Tang, K.Z., and Detre, J.A. (2006). Continuous ASL perfusion fMRI investigation of higher cognition: quantification of tonic CBF changes during sustained attention and working memory tasks. Neuroimage 31, 376-385. 
Koric, L., Volle, E., Seassau, M., Bernard, F.A., Mancini, J., Dubois, B., Pelissolo, A., and Levy, R. (2011). How cognitive performance-induced stress can influence right VLPFC activation: An fMRI study in healthy subjects and in patients with social phobia. Hum Brain Mapp.

Langdon, D.W. (2011). Cognition in multiple sclerosis. Curr Opin Neurol 24, 244-249.

Lewin, J.S., Friedman, L., Wu, D., Miller, D.A., Thompson, L.A., Klein, S.K., Wise, A.L., Hedera, P., Buckley, P., Meltzer, H., Friedland, R.P., and Duerk, J.L. (1996). Cortical localization of human sustained attention: detection with functional MR using a visual vigilance paradigm. J Comput Assist Tomogr 20, 695-701.

Lewis, J.W., Frum, C., Brefczynski-Lewis, J.A., Talkington, W.J., Walker, N.A., Rapuano, K.M., and Kovach, A.L. (2011). Cortical network differences in the sighted versus early blind for recognition of humanproduced action sounds. Human brain mapping 32, 2241-2255.

Lim, J., Wu, W.C., Wang, J., Detre, J.A., Dinges, D.F., and Rao, H. (2010). Imaging brain fatigue from sustained mental workload: an ASL perfusion study of the time-on-task effect. Neuroimage 49, 34263435.

Lim, J., Catherine-Quevenco, F., \& Kwok, K. (2013). EEG alpha activity is associated with individual differences in post-break improvement. NeuroImage. doi:10.1016/j.neuroimage.2013.03.018

Mantini, D., Perrucci, M.G., Del Gratta, C., Romani, G.L., and Corbetta, M. (2007). Electrophysiological signatures of resting state networks in the human brain. Proc Natl Acad Sci U S A 104, 13170-13175.

Mckeown, M.J., Makeig, S., Brown, G.G., Jung, T.P., Kindermann, S.S., Bell, A.J., and Sejnowski, T.J. (1998). Analysis of fMRI data by blind separation into independent spatial components. Hum Brain Mapp 6, $160-188$.

Nobre, A.C., Sebestyen, G.N., Gitelman, D.R., Mesulam, M.M., Frackowiak, R.S., and Frith, C.D. (1997). Functional localization of the system for visuospatial attention using positron emission tomography. Brain 120 ( Pt 3), 515-533.

Ogg, R.J., Zou, P., Allen, D.N., Hutchins, S.B., Dutkiewicz, R.M., and Mulhern, R.K. (2008). Neural correlates of a clinical continuous performance test. Magn Reson Imaging 26, 504-512.

Pfurtscheller, G., Stancak, A., Jr., and Neuper, C. (1996). Event-related synchronization (ERS) in the alpha band--an electrophysiological correlate of cortical idling: a review. Int J Psychophysiol 24, 39-46.

Schrauf, M., Sonnleitner, A., Simon, M., and Kincses, W.E. (2011). EEG Alpha Spindles as Indicators for Prolonged Brake Reaction Time During Auditory Secondary Tasks in a Real Road Driving Study. Proceedings of the Human Factors and Ergonomics Society Annual Meeting 55, 217-221.

Smith, S.M., Fox, P.T., Miller, K.L., Glahn, D.C., Fox, P.M., Mackay, C.E., Filippini, N., Watkins, K.E., Toro, R., Laird, A.R., and Beckmann, C.F. (2009). Correspondence of the brain's functional architecture during activation and rest. Proc Natl Acad Sci U S A 106, 13040-13045.

Sonnentag, S., and Zijlstra, F.R.H. (2006). Job characteristics and off-job activities as predictors of need for recovery, well-being, and fatigue. Journal of Applied Psychology 91, 330-350.

Tamaki, M., Shirota, A., Tanaka, H., Hayashi, M., and Hori, T. (1999). Effects of a daytime nap in the aged. Psychiatry Clin Neurosci 53, 273-275.

Uddin, L.Q., Kelly, A.M., Biswal, B.B., Xavier Castellanos, F., and Milham, M.P. (2009). Functional connectivity of default mode network components: correlation, anticorrelation, and causality. Hum Brain Mapp 30, 625-637.

Vahdat, S., Darainy, M., Milner, T.E., and Ostry, D.J. (2011). Functionally specific changes in resting-state sensorimotor networks after motor learning. The Journal of neuroscience : the official journal of the Society for Neuroscience 31, 16907-16915.

Valdez, P., Reilly, T., and Waterhouse, J. (2008). Rhythms of Mental Performance. Mind Brain and Education 2, 7-16.

Van De Ven, V., Esposito, F., and Christoffels, I.K. (2009). Neural network of speech monitoring overlaps with overt speech production and comprehension networks: a sequential spatial and temporal ICA study. Neuroimage 47, 1982-1991.

Van De Ven, V.G., Formisano, E., Prvulovic, D., Roeder, C.H., and Linden, D.E. (2004). Functional connectivity as revealed by spatial independent component analysis of fMRI measurements during rest. Hum Brain Mapp 22, 165-178. 
Van Den Heuvel, M.P., Mandl, R.C., Kahn, R.S., and Hulshoff Pol, H.E. (2009). Functionally linked restingstate networks reflect the underlying structural connectivity architecture of the human brain. Hum Brain Mapp 30, 3127-3141.

Van Der Linden, D., Frese, M., and Meijman, T.F. (2003). Mental fatigue and the control of cognitive processes: effects on perseveration and planning. Acta Psychologica 113, 45-65.

Waites, A.B., Stanislavsky, A., Abbott, D.F., and Jackson, G.D. (2005). Effect of prior cognitive state on resting state networks measured with functional connectivity. Hum Brain Mapp 24, 59-68.

Worden, M.S., Foxe, J.J., Wang, N., and Simpson, G.V. (2000). Anticipatory biasing of visuospatial attention indexed by retinotopically specific alpha-band electroencephalography increases over occipital cortex. J Neurosci 20, RC63.

Zhou, Y., Milham, M.P., Lui, Y.W., Miles, L., Reaume, J., Sodickson, D.K., Grossman, R.I., and Ge, Y. (2012). Default-mode network disruption in mild traumatic brain injury. Radiology 265, 882-892.

Zijlstra, F.R.H. (1993). Efficiency in Work Behavior: A Design Approach for Modern Tools. Delft: Delft University Press.

Zuo, X.N., Kelly, C., Adelstein, J.S., Klein, D.F., Castellanos, F.X., and Milham, M.P. (2010). Reliable intrinsic connectivity networks: test-retest evaluation using ICA and dual regression approach. Neuroimage 49, 2163-2177. 

Chapter 7

\section{Discussion}


CHAPTER 7 
In the previous chapters, I have presented three studies that are, to my knowledge, unique in their approach to investigate the neural correlates of mental effort. For the first time, it was explored how mental effort investment is experienced, evaluated and which consequences it has on functional networks in the human brain. The unique aspect of this work is that the subjective experiences of the participants in terms of mental effort were measured by a proven instrument while simultaneously measuring correlates of brain activation. Thereby, it was possible to quantify the influence of our changing experimental conditions on the participants' experience and their neural processes at the same time.

In doing so, we aimed at solving fundamental, but also practical questions about mental effort. Is experiencing mental effort handled by the same neural systems that are recruited in its later evaluation by an operator? Is it feasible to assume that post-performance self-ratings reflect the experience of an operator during performance? If mental effort is a costly, finite resource, how does this affect brain functioning?

In particular, we aimed to answer three questions:

- Which neural mechanism underlies the subjective post-performance selfevaluation of mental effort investment?

- Does this mechanism reflect the experience of mental effort investment during task performance?

- Can we find measurable differences in brain functioning that result from sustained mental effort investment?

Our understanding of mental effort is reflected in the tools that we use to measure it in laboratory and real-world situations. In using subjective self-report tools, we assume that the operator can give an accurate account of his or her experience at a later point, using an instrument such as the RSME to quantify a subjective firstperson impression. In other words, we assume that the process of remembering and evaluating one's previous effort investment brings back an accurate memory of the experience. We also assume that changes in effort correspond to a coherent construct, irrespective of the question if a task-sided or operator-sided factor caused the change in effort requirement. The first step in evaluating this assumption was to identify the neural structures which are recruited during effort evaluation. As a second step, we needed to test if these neural structures have an overlap with structures that are sensitive to changing levels of effort investment while a participant performs a task. Finding that neural structures recruited during effort evaluation are different from structures that are sensitive to effort investment during performance would rather suggest that the two processes do not rely on similar computational resources in the brain. While this would not automatically condemn the validity of self-ratings of mental effort in general, it would constitute that selfratings are a less direct measure of the subjective experience as previously thought. A similar case would be finding that the neural structure would for example be 
reactive to changes in effort due to one class of loads, but not another, i.e. being only sensitive to changes in task-, but not in state load. A finding like this would argue against an instrument such as the RSME which measures mental effort as one coherent concept irrespective of the fact that task load and state load pose different sources of alterations in mental workload.

Other domains of subjective experience have yielded exactly such separations in their neural correlates: The experience of pain, for instance, is processed in the human brain in two networks which predominantly are concerned with predominantly the sensory-discriminant or with the affective/attentional component of the pain experience (Peyron, Laurent, \& Garcia-Larrea, 2000). This prompts the conceptual question if the subjective experience of pain can be seen as a coherent construct, or if sensory and affective components should be viewed as related, but separable aspects of the pain experience.

Last, but maybe most important, our fundamental understanding of mental effort suffers from the lack of a concrete and measurable resource. This assumption of a finite resource that "fuels" effortful self-regulation is central to modern cognitive models such as the integrated model of mental effort (Mulder, 1986), or the strength model of self-control (Baumeister, Heatherton, \& Tice, 1994).

An important part of the concept is that the resource is finite, and thus an operator will not be able or willing to invest it endlessly. Sustained effort investment increases the amount of effort that is required to guarantee performance (OdleDusseau, Bradley, \& Pilcher, 2010). If the amount of required effort is larger than what the operator is willing to invest, he or she will disengage from the task (Richter \& Gendolla, 2007). As the last study of this project, we used a paradigm involving sustained effortful activity, in order to see how changing levels of mental effort "fuel" affects brain functioning.

As the next part of this chapter, I briefly summarize the three studies that we conducted and relate their findings to the overarching questions that prompted this project. Next, extending the individual discussions of these findings in the respective chapters, I relate the individual results to the understanding of self-report instruments such as the RSME and to the underlying theoretical considerations and assumptions. In the last part of this chapter, I elaborate on the implications that not only the results, but also the approach of this project have for the further advance of occupational psychology.

\subsection{Summaries of the conducted studies}

\subsubsection{Study 1}

In the first study of this project, described in chapter 4, we investigated which brain areas became differentially activated when participants rated the difficulty and 
their effort investment on a mental task using subjective self-report instruments. Self-evaluation instruments enable the individual to provide reliable estimates of his or her mental effort investment related to performing a task.

The design of this study build on results from earlier research, in which it was demonstrated that the ability to report mental effort investment is dissociable from similar self-ratings (Naccache et al., 2005). The evidence underlying this finding consisted of a case report into one patient, abbreviated RMB. Following a brain lesion, RMB was neither able to experience her own effort investment, nor to perform self-evaluations of mental effort investment. However, she could without problems deduct and evaluate the difficulty of a task from overt task characteristics.

The combination of functional loss of both of these capabilities in patient RMB constituted evidence in favor of common neural substrates for experiencing and self-evaluating mental effort. However, the case of patient RMB also demonstrated that the neural processes related to mental effort are apparently dissociable from other, similar processes, as RMB was able to deduce changes in task difficulty. This illustrated that the acts of rating the difficulty of a recently performed task and rating the amount of effort needed to perform this task are not identical in their reliance on neural information processing. A demonstration of a functional dissociation of two processes in a lesion case like this forms an important begin in relating functions and neural structures. Alas, such patient studies only represent isolated cases, as the sustained lesions sustained by single patients are mostly extensive and not defined in their location. Hence, it is in most cases not possible to establish a generalizable relation between a specific functionality and a neural structure on the basis of observations like this one. However, this case showed that apparently, two constructs as highly related as difficulty and effort do not rely on identical neural correlates. In our first study, we used this observation to isolate brain areas which become more activated during mental effort self-evaluation in healthy participants. For this, we had participants perform a working memory task on different levels of difficulty. After each short block, participants had to perform self-ratings of perceived task difficult and perceived effort investment. The results showed that in particular the left anterior insular cortex (laIC) was activated stronger during selfevaluation of effort expenditure when compared to an evaluation of task difficulty. The anterior insular cortex has been proposed to integrate cognitive, emotional and somatic information to form a coherent representation of the state of the self (Craig, 2009). Evidence from a study by Modinos, Ormel and Aleman (2009) provides further evidence for a functionality of laIC related to self-related evaluations by showing that this region becomes more activated during evaluation of statements about oneself than during evaluation of statements about peers.

Following the view of Craig (2009) we interpret the increased activation of laIC during mental effort evaluation as a sign that this type of evaluation relies on the successful integration of somatic, emotional and cognitive information related to the state of the self. It has been proposed earlier that such an integration of infor- 
mation from different domains is necessary in order to perform self-evaluations of mental effort investment: The original interpretation by Naccache et al. (2005) of their patient's inability to perform self-evaluations of effort investment focused on the effect of her lesion on the neural system mediating the propagation of somatic information. The lesion of patient RMB disturbed the generation of somatic markers in her brain. Somatic markers signal changes in physiological parameters of the body and are used to relate cognitive information to emotions (Damasio, 1999). Without somatic markers, patient RMB was not provided with information about the effects that performing a task had on her own state. As Naccache et al. have elaborated, this lack of information lead to patient RMB being unable to experience or evaluate her own effort expenditure.

Our results support this interpretation by showing that the self-evaluation of mental effort leads to increased activation in the laIC as a neural structure that is assumed to integrate somatic, emotional and cognitive information. We interpret the recruitment of this structure as evidence for the need to integrate these different categories of information in order to perform a self-evaluation of mental effort investment. Logically, if one of the information categories is missing, the integration cannot be performed.

Apart from this, our results suggested a further role of laIC during performance itself: While the participants performed the task at different levels of difficulty, the signal change in the laIC cluster varied roughly in the same pattern as the RSME scores that the participants provided directly after each block of task performance. This suggested that laIC not only serves a role in the later evaluation of effort expenditure, but also monitors it during performance.

\subsubsection{Study 2}

The observation of parametrical modulations in laIC activation had an important implication for solving the second question. This question referred to an important assumption behind self-rating measurement tools for mental effort, namely that the evaluation of one`s effort investment after task performance equals the experience of mental effort investment during task performance.

Patient RMB`s combined inability to both experience her investment of mental effort and to provide an evaluation of it at a later point already supported the assumption of a relation of experience and later evaluation. Finding now that a neural structure that is activated particularly strong during mental effort self-evaluation displays task-load sensitive changes in activity during task performance is further evidence in favor of a common mechanism for the experience and off-line evaluation of mental effort investment. The paradigm that we used in study 1, however, did not allow making a statement about the role of laIC in the acute experience of effort investment. In study 1, we only manipulated the task difficulty. Variations in difficulty on the same task did by no means only affect activation in laIC during task performance, but a lot of other frontal and parietal areas. This finding is common in 
working memory tasks as the n-back task that we used here (Owen, McMillan, Laird, \& Bullmore, 2005), but also for other mental tasks (Duncan \& Owen, 2000). It is not easily possible to separate the changes in activation due to increased processing from the changes in activation due to a change in the experienced amount of mental effort investment. Furthermore, task load only forms one factor in the total mental workload that participants face. If a neural structure is sensitive to workload as such, instead of merely reflecting the level of task load, it has to react to changes in both task and state load. Our results indicated that laIC reacts to changes in task load, but would it also react to changes in state load? To answer this question, we had to take a closer look at brain activation during task execution. From the literature and also from our first study, we knew that only varying the task demands would affect a large number of brain areas. This would include all areas that simply become more active to process the additional amount of information the "muscles" of the brain, so to say. Areas that would be modulated due to changes in effort would be difficult to separate from these wide-spread activation differences. Thus, in our second study, described in chapter 5 , we used an extension of our original design form study 1 to solve this problem. Study 2 investigated the supposed role of laIC during task execution. Again, we used short blocks of n-back tasks with varying task load. But instead of only manipulating task difficulty, also the state of the subjects was manipulated. This was achieved by having subjects spend the day with an effortful activity or leisure time before having them perform, again, an n-back working memory task while we measured their brain activation. This time, however, we were not interested in differences between different kinds of self-ratings; rather, we were interested in which brain areas activity would be influenced in the same way by changes in task and state load as the subjective ratings of effort investment. The RSME scores obtained after task performance showed an interaction of task difficulty and the state of the subjects. This means that after a day of effortful activity, the differences in task difficulty affected the subjectively experienced amount of effort investment differently: While the total amount of effort needed was increased, the difference between the most easiest and hardest n-back conditions became smaller, as even performing the simple 1-back condition required the participants to invest effort in regulating their state, adding to the effort they had to invest purely into the task as such. When analyzing the fMRI data taken during task performance, the same interaction effect was found in laIC. This shows that indeed, laIC reacts on factors which influence the amount of mental effort that a subject needs to invest to perform a task. More important, both taskand subject-sided sources of demand change where influential. This demonstrates clearly that the changes in activity observed in laIC are not simply related to changing levels of information processing load mediated by the different n-back conditions. Thus, next to being more active during the post-task evaluation of mental effort expenditure, laIC is apparently also involved in its experience during performance. This is the first time that any central variable has been shown to react to 
changing demands of mental effort that are mediated by both changes in task load and in state load.

It is known from the literature that the bilateral laIC, as well as its right counterpart are involved in the representation of task goals and strategies (Dosenbach, Fair, Cohen, Schlaggar, \& Petersen, 2008), but also in monitoring performance (Nelson et al., 2010). One factor setting laIC apart, though, is that it is the target site of heartbeat-evoked-potentials (HEP) (Gray et al., 2007). Measures of heart rate have historically been used to indicate levels of elevated mental workload (Nickel \& Nachreiner, 2003), which, given that the operator accepts this increased load, results in increased mental effort investment. As Nickel and Nachreiner (2003) note, heart rate is not a very specific indicator of mental workload changes, as it is influenced by numerous other processes in the human body. In our interpretation, information about myocardial functioning is one of several information components that must be integrated to derive an indication of the amount of invested mental effort.

In the emerging picture of the laIC's role in effort management, we thus have the prior knowledge that laIC is supplied with information that needs to be integrated in order to evaluate the impact of mental effort investment on the state of the individual, not only in cognitive, but also in emotional and somatic terms (Craig, 2009; Gray et al., 2007).

We also have at least the isolated observation from patient RMB that a failure to integrate the state-related information leads to both the inability to experience effort investment, but also to the inability to evaluate it at a later point. Taking now the results from study 2 , in which we find that a cluster in laIC is modulated by changes in workload during task performance in the same fashion that the RSME is modulated by these changes after performance, we conclude that laIC plays a crucial and common role in both the acute experience of effort investment and its later off-line evaluation. This carries important implications for the second question behind this project, the assumption of a commonality of experience and later selfevaluation of mental effort. Although we have no direct way of objectifying the subjective experience of a participant, we do have evidence which shows that the same cortical area is implied in experience and later self-evaluation of mental effort. Also, we show that this area reacts in the same way to changes in task- and state load during task performance as the closest available indication of subjective experience, the RSME, shortly thereafter. In our interpretation, this evidence supports the assumption that the RSME scores given after task performance reflect the experienced amount of acute mental effort investment during performance.

\subsubsection{Study 3}

The third and last study of this project investigated changes in the resting state networks in the brains of subjects as an effect of depleting mental resources. This study, described in chapter 6 , was conducted to solve the question if the depletion of the mental resources of a person would correspond to changes in functional 
networks of brain areas. The integrated model of mental effort assumes an abstract resource as the crucial, yet finite factor driving regulations of state and information processing. A depletion of this resource leads to a progressive increase of the amount of effort necessary to perform an otherwise unchanged task. This is mainly reflected in the impairment of controlled attention and controlled processing, usually accompanied by a feeling of exhaustion. To induce such depletion, subjects spend several hours performing an effortful task. As a contrast, subjects spent the same time with low-effort, self-chosen activity. When comparing the measurements taken in the afternoon, the effortful day lead to a number of changes both in the subjective self-reports provided by the subjects and in the functional networks of their brains. The results showed an increase in exhaustion and an increase in subjectively rated mental effort expenditure on a working memory task after the effortful day. At the same time, functional connectivity in a network associated with executive functioning was decreased, indicating reduced efficiency in controlled information processing. Also, we observed increased coherence in the default mode network and the early visual network. Increases in default mode network are associated with a tendency for unguided, intrinsic attention, while increases in coherency of the early visual network have been linked to inhibition of visual processing. Furthermore, in all three of those networks, there were also more regionally specific effects that correlated with the subjective exhaustion scores. This could be shown by correlating the regional independent component analysis (ICA) scores (see box 1) with the subjective exhaustion ratings. The ICA score of a brain area in the network related to executive functioning was negatively correlated with exhaustion, suggesting that rhythmic fluctuations of activity in this area was more out of sync with the rest of this functional network when participants were more exhausted. In the early visual network and the default mode network, several regions` ICA scores correlated positively with exhaustion. These results show that the implementation of specific areas in functional networks can be modulated by sustained effort investment. Our experimental treatment had thus not only a measurable effect on functional networks of brain regions, but this effect was correlated to the participants` self-evaluation of their own state in terms of exhaustion. Again, these changes were accompanied by a significant increase in effort expenditure to maintain performance during a separate working memory task. Although the precise cause for these changes in the functional networks is not yet known, they represent a physiological manifestation of the effect of depleting the still-abstract resource underlying mental effort. This finding will be discussed more detailed in the general discussion, as it yields implications for the conceptualization of mental effort in general. 


\section{Box 1: Understanding ICA scores}

To understand ICA scores, start by comparing the brain to a restaurant full of people. Imagine that we would know nothing about the way a restaurant is organized. To make sense of how this restaurant functions, we take a look at the staff. Pretend for the sake of simplicity that this restaurant features an open kitchen, giving us an overview of pretty much all the staff. The restaurant in our example has a very friendly atmosphere, thus the greeters guide patrons to their tables, and the cooks check up on the reception of their food, so we cannot simply deduct the function of staff members from their location. The first thing that we notice is that staff members wear different uniforms. The greeters wear a different suit than the waiters, and both are easily distinguished from the cooks in their white aprons. Imagine now that all the staff members would wear the same outfit. How would you still be able to put different staff members into a category? One way is to look at their coordinated activity. If a party of patrons arrives, the greeters work in a coordinated way to welcome the guests, take the coats, and lead the patrons to their table. When a course for this party is ready, a swarm of waiters will hurry to serve all plates simultaneously. If we thus see a waiter running with a plate, we will be able to predict that other members of the same staff category will also be running at the same time. We can thus say that our observation of the initial waiter yields information that can be used to predict the behavior of other waiters. Thereby, we have identified one component within the staff. Also, we can make a difference between members of this staff component and another staff component by observing that the behavior of a waiter does not yield predictive information about greeters. We thus conclude that these two staff components are independent. The same approach can be used on brain data; the total mass of voxels can be split in independent groups, or components, based on the fact that the behavior of voxels in one component does not yield information that can be used to predict the behavior of a voxel in another component. The ICA score, then, represents how much a voxel is in phase with the other voxels in this component. Looking back at our restaurant example, a waiter who moves slightly out of sync with his fellow waiters would have a low ICA score. In study 3, we averaged the ICA scores of the single voxels of a component that were also spatially clustered together, meaning that these voxels were in the same brain area. In that sense, voxels that are clustered together would represent one member of the staff. Thus, from one staff component, we derived several staff members, or clusters. The behavior of these clusters in terms of how much their activation changes are in phase with the other clusters is thus expressed in regional ICA scores. 


\subsection{General discussion}

\subsubsection{Neural correlates of mental effort and their implications for self-rating instruments}

The three studies that we conducted during this project represent the first step towards a more thorough understanding of the neural processes that take place during the performance of effortful mental activities.

Evidence from our first two studies has demonstrated a central role of laIC in mental effort investment. When we take evidence from earlier research into the functionality of laIC into account, a picture starts to emerge in which laIC apparently forms a neural junction fit to monitor and control effort investment. Several studies have shown that the laIC, but also its right counterpart, perform a role in the representation of the goals of a task, and also of the strategies that operators apply (Dosenbach et al., 2008); Fair et al., 2007). Also, Nelson et al. (2010) have shown that, additionally, left and right aIC integrate performance-related information during task performance. Thereby, both aICs are involved directly in the execution of mental tasks. Although the actual information processing that needs to take place has been shown to be handled in a variety of areas, the aICs take more of a managerial role. Our own evidence supports this interpretation and extends it: At least in case of one aIC, namely the laIC, the managerial function extends to monitoring effort investment and to bringing effort investment to the awareness of the operator.

Finding evidence in support of a common neural basis for experiencing and evaluating mental effort has direct implications for the fundamental assumption behind self-rating instruments such as the RSME. In our understanding of these instruments, there has always been a major gap, caused by the lack of a possibility to evaluate the construct validity of the instruments in a more objective manner. In case of the RSME, but also in general with subjective self-report instruments, the measurement scores that are collected reflect a more or less abstract indication that a participant gives about his or her first-person experience. The crucial point in the case of mental effort is that we cannot, with any other psychological tool, get closer to the first-person-experience of the participant. This experience is, so to say, the at the most inner core of the psychological construct that we want to measure. Between the experience and any measurable manifestation such as related behavior (e.g. performance in the case of mental effort), there are numerous other processes which all pose sources of interference. Thus, it is imperative to ensure that the instrument measures the construct that it is intended to measure. Meeting this criterion of construct validity can be difficult in case of instruments measuring subjective experiences. These difficulties affect design and validation of instruments alike: Even when self-report instruments are tested under laboratory conditions, the same experimental manipulations can affect several aspects of the subjective 
experience in participants. For example, manipulating the demands of a task will affect both the perceived difficulty of a task and the perceived amount of effort investment necessary to perform the task. Measures of difficulty and mental effort investment have been observed to be highly correlated in the past, and this observation has even been interpreted previously as a sign that the measurement instruments of difficulty and of effort refer to highly similar constructs (Yeo \& Neal, 2004). Without any closer indicators of mental effort than self-ratings and the even more indirect performance output of the participant, it is not trivial to evaluate if an instrument measures one or the other construct. Task load is an integral part of the construct of mental effort, as elaborated in the introduction of this volume. Thus, depending on the experimental paradigm that is used, it can be impossible to separate subjective experiences of effort and task difficulty. The observations in patient RMB demonstrated that there apparently is a difference in the neural processing structures of effort and difficulty. This initial observation enabled us to successfully identify the laIC as a neural structure that reacts different during evaluations of difficulty and effort. In order to investigate if laIC would be influenced by changes in experienced effort and not just by changes in difficulty, we used manipulations of task and state load, similar to the ones used to influence the experienced amount of invested effort in participants during the validation research for the RSME. The original validation procedure of the RSME included the manipulation of both the task load and the state load by varying not only the processing complexity of a task, but also by testing participants after a free day and a working day.

Our results show that the activation of laIC during task execution is influenced both by changes in task load and also by the interaction of task load and state load. The related ratings that participants provided using the RSME showed corresponding changes in the reports of subjectively experienced mental effort investment. We were thus able to demonstrate that the subjective ratings of participants on the RSME and laIC activation in their brains react in a similar way to changes in task load and state load. Thus, the way that the RSME reflects the subjective experience of an operator's mental effort investment is similar to the way that a neural structure implied in the management of mental effort is modulated in its activity. This allows us to propose that the RSME offers operators a quite direct way to express their experienced amount of mental effort investment, free of any systematic distortion.

Yet, the observation of a common effect of task- and state load manipulations on the same neural structure also allows to make additional statements about the RSME`s rationale of measuring one single dimension of mental effort. The fact that manipulating subjectively experienced mental effort investment relates to changes in one single brain region also provides support for the rationale of the RSME in which effort is measured as one coherent experience. In this approach, the RSME stands in contrast to other instruments such as the SWAT (Reid, Eggemeier, \& Shingledecker, 1982) or the NASA-TLX (Hart \& Staveland, 1988), which aim to capture different aspects of effort investment by including multiple rating items. As already 
noted by Zijlstra (1993), at least the studies using the 6-dimensional NASA-TLX reported high correlations between different sub-scales of the instrument. This was one of the main factors, next to considerations such as usability, which lead Zijlstra to favor a one-dimensional approach when constructing the RSME. Our findings, in which we demonstrate that changing the experienced amount of mental effort with both state- and task load manipulations manifests in the same neural structure, pose evidence in favor of this approach. Considering the neural processes underlying apparently simple self-reports of a psychological concept have the potential to change our understanding of those very psychological concepts. Research into other subjective ratings such as measurements of experienced pain have for example shown that the seemingly uniform concept of pain is, in fact, not uniform at all: Different aspects of a painful experience have been shown to be processed in different neural systems, which made it plausible to differentiate between sensorydiscriminant and affective/attentional components of the pain experience (Peyron, Laurent, \& Garcia-Larrea, 2000).

In the case of mental effort, our results constitute evidence in favor of a concept of mental effort in which effort is one coherent resource that is consumed by meeting task load and state load alike. The laIC as a brain region that is recruited selectively stronger during mental effort self-evaluation was shown to be affected by both changes in task load and in state load alike during acute effort investment. Our study thus continues the example of earlier work in underlining how cognitive models of subjective experiences and the associated measuring instruments can benefit from a more thorough investigation of their neural correlates.

\subsubsection{Rethinking the energetical resource assumption}

Arguably the most fundamental contribution that our project has made in advancing the concept of mental effort is related to the last and most challenging question that stood at the beginning of this endeavor. The concept of mental effort describes how a limited resource is invested in order to regulate processing, behavior or even the fundamental state of an individual. By assuming that this resource is necessary for all regulative processes, but at the same time finite, it is implicitly stated that spending this resource has a profound influence on the individual, or more precisely on the functioning of the brain. While the precise nature of this resource remains unknown, our results open the possibility for a potential paradigm shift in conceptualizing it. As shown, the depletion of mental resources by sustained effort investment had measurable effects on the functional connectivity of brain areas. It was shown that these changes in functional connectivity correspond to the need of investing an increased amount of mental effort in task that objectively did not change in its demands. Our results thus demonstrate a close relation of the concept of mental effort to measures of the brain`s functional organization. Even more, the measurements of functional connectivity that we report form, for the moment, the most direct and objective correlate of mental effort. While earlier attempts into finding a 
physiological variable have largely focused on identifying a correlate of acute or recent effort investment such as HRV components (Mulder, 1980) or blood glucose levels (Gailliot et al., 2007; Scholey, Harper, \& Kennedy, 2001), our approach links the effect of mental effort investment to brain functioning in a much more direct way.

The challenge posed by both the integrated model of mental effort (Mulder, 1986) and the strength model of self-control (Baumeister et al., 1994) was to use physiological measures to identify the nature of the resource that underlies effortful regulation. Our results open the way for considering a radically new view of mental effort as a resource. Traditionally, effort has been pictured as an energetical resource. Based on our results, we propose a different perspective: What if the concept of mental effort does not relate to an energetical resource, but to the normal, ideal harmony of the brain, in which functional networks of brain regions cooperate in an efficient manner?

Recent studies into the functional organization of brain areas have consistently shown two findings which are related to this interpretation: First, the functional organization of brain areas is a very delicate process. Functional networks of brain areas operate in a flexible manner to offer the required processing while minimizing operational costs in form of glucose and oxygen (Bullmore \& Sporns, 2012). The second consistent finding is that a large number of neurological diseases and injuries that are associated with a decrease of mental capabilities are accompanied by changes to functional networks (Van Den Heuvel \& Hulshoff Pol, 2010; Stam \& van Straaten, 2012). Recent evidence from a clinical study even demonstrated a significant correlation of improving connectivity in the right fronto-parietal network (FPN) after surgical treatment of invasive tumors, and cognitive abilities of the patients (van Dellen et al., 2013). The FPN forms one of the brains executive networks, and our own results showed a widespread reduction in right FPN coherence as an effect of sustained, effortful performance. It seems thus warranted to assume that any changes in the normal balance of functional connectivity can manifest in an increased need to invest regulative resources in order to guarantee performance. In the view proposed here, the very act of investing mental effort to sustain performance actually disturbs the functional harmony in which brain areas can form flexible, effective networks in the most efficient way. Thereby, homeostatic balance itself would form a resource, which can be seen as being invested. This view of mental effort as a homeostatic instead of an energetical resource is compatible with the integrated model of mental effort (Mulder, 1986) and the strength model of self-control (Baumeister et al., 1994): Although these models depict a reservoir of effort, this reservoir could equally well represent an overall indicator of the homeostatic status of the brain in terms of functional organization. Regulative action would affect this reservoir by de-balancing the delicate overall balance of this functional organization, thereby increasing the need for regulative action further on throughout the course of sustained performance. 
There are two immediate open questions which need to be solved to evaluate our proposed homeostatic concept of mental effort. One, it remains unclear by which mechanism regulative action affects the delicate balance of functional organization. Two, it is far from sure if the proposed disturbance in functional organization is the only factor that plays a role in an increased need for regulative action in the case of sustained performance. Both of these issues could very well be related to functional changes on a neuronal level as an effect of sustained performance. One plausible mechanism for such a localized reduction in function might be the depletion of local glucose resources. Evidence from animal studies has demonstrated that the local level of glucose in the extracellular fluid surrounding task- relevant neurons can decrease as an effect of cognitive load (McNay, Fries, \& Gold, 2000). A further investigation of the local mechanisms of neuronal supply in humans might thus lead to an additional, actually energy-related factor in our understanding of mental effort.

\subsubsection{Implications and outlook}

The studies conducted in this project form a step forward in the exploration of occupation-related phenomena using neuroimaging methods. While task-specific processes in the brain have been investigated to a greater extend, more general aspects of goal-directed performance ("work") are just now becoming a subject of interest.

One aspect setting this work apart is the novel approach of using neuroimaging methods next to the known instruments used in occupational psychology. This approach has opened the possibility to understand the fundamental processes underlying the interaction of a person with a task. In the case of mental effort, this regards an aspect that is universally present in every task-person interaction, professional and casual. The studies presented in this book mainly concerned three aspects of mental effort investment: The self-evaluation of invested effort, changes in the amount of experienced effort as an effect of the factors described in the integrated model of mental effort; and the changes that can be observed as a consequence of sustained effort investment. These aspects arguably form the most relevant components of the subject of mental effort when looking at the applied side of occupational psychology. Self-rating instruments as the RSME are used in the field to determine the amount of effort that is invested in task performance. Using these measurements, the interaction of persons and tasks is optimized to provide an optimal workload while ensuring safe performance. The depletion of resources forms one of the most detrimental factors leading to human error, with all consequences that may entail. While factors in the person-task-interaction have been identified which are influential for the outcome of this interaction, the underlying processes in the human brain were not well understood. By exploring the neural substrates of these three aspects of mental effort, the first step has been made to further understand how functions in the brain contribute to the interaction of task and operator. 
In the case of mental effort, the most plausible continuation of this exploration would be to study the mechanisms behind the experience of mental effort expenditure. How do single factors - in the task or in the person - influence the total experience of effort investment? Although the results in this study feature the laIC prominently, it can be speculated that this region is only a part of a larger network of brain regions that influence this experience. Studies involving an analysis of the functional connectivity of laIC with other regions could give more information about the determinants of effort experience. An exciting next step would be to find neurobiological relations between laIC and brain structures implied in motivation, or laIC's eventual connection to neural systems that are connected to reward. The investment of mental effort does not happen in a vacuum, of course, and understanding how the neural correlates of mental effort are related to the neural correlates of processes that are closely connected to it potentially yields a better holistic understanding of how effortful performance is integrated in the total (neural) functioning of an individual. One can assume that the sustained investment of mental effort, as it happens in the case of work, has a profound effect on the individual over time, as a substantial period of our waking adult life is spent on occupational activities. It has been shown that such activities carried out over a long time can change brain functioning in a profound way. This can go as far as to lead to structural changes in the brain, as for example observed in cab drivers (Maguire, Woollett, \& Spiers, 2006). London cab drivers, when compared to London bus drivers, were found to have a greater gray matter volume in parts of the hippocampus, which the authors attribute to an extensive memorization of London`s street maps. Bus drivers, who in theory have a very similar occupation, follow fixed routes. Such a seemingly minor difference in the job characteristics of a person`s occupation can thus influence brain functioning not only on a transient level, as the effect of sustained performance that we have shown in study 3 , but can even result in stable, structural changes in the brain. Such changes have the potential to influence a person`s functioning: While some parts of the hippocampus where enlarged, other parts showed less grey matter volume, and the authors suggest that this reduction might be the cause of cab drivers performing worse on a neurological learning test.

This particular observation concerned the effects of highly specialized tasks on the functionally specialized brain structures which support them. However, it can be argued that also more general characteristics of the person-task-interaction will have a profound influence on brain functioning. While the specific content of the tasks performed during our working life may change, our strategy to approach them and experience our investment of effort can remain the same. Evidence even shows that this interaction is influenced by our stable personality traits (Yeo \& Neal, 2008). The effects of a long term exposure to a certain pattern in this interaction can be supposed to have dramatic influence on several aspects of a person`s life, manifesting themselves for example in the many adverse psychological and physiological effects of work stress on mental (Juster, McEwen, \& Lupien, 2010) and physiological health (Chandola et al., 2008). By understanding the way that the 
brain interacts with the challenges, more possibilities to influence this interaction in a beneficial way are likely to emerge. To strike a comparison, the field of occupational medicine has made great advances in developing guidelines that help to shape the interaction of the body and the task challenges in an ergonomic way. This was only possible by the advances in medical knowledge and techniques, which made it for example possible to explore the etiology of musculoskeletal disorders related to work. In the same way, it has become possible to explore the functions of the brain in relation to work with the modern neuroimaging methods that are now at our disposal. These methods can extend the view of occupational psychologists in the same way that x-ray and MRI extended the view of occupational physicians. Occupational psychology is unique in its combination of theoretical, cognitive aspects on the one hand and the applied side on the other. Combining this unique expertise with neuroimaging methods, creating a so-called "occupational neuroscience", would extend its reach dramatically. In the end, the main goal is to foster the mutually beneficial interaction of a person and a challenging environment. This approach extends the existing direction of Neuroergonomics (Parasuraman, 2003) in the sense that it does not only aim at fostering the direct improvement of interfaces based on the knowledge of the implementation of cognitive processes in the brain. Occupational neuroscience adapts a rather broad scope in which the implications of the "brain at work" are considered in the larger context of the effects of work on the individual. Without dispute, the potential benefits of interfaces following the ergonomic principles of brain functioning are most promising. Yet, such measures form just a part of the possibilities that are opened up by utilizing the broad knowledge base of occupational psychology. These possibilities extend far beyond the direct work environment. They literally begin in the pre-adolescent training of the individual and follow it beyond the workplace, to recovery and to other domains of life, and by leaving a lasting effect on the brain, into retirement. Occupational neuroscience opens the possibility to educate individuals about the principles that connect the way they use their brains at work to the way that their brains, in turn, shape their conscious experience. The capability to regulate oneself, one`s affective state or motivation are just the most obvious aspects that are influenced by the one`s way to face an effortful task. Realizing the implications and consequences of one`s way to approach a mentally demanding task, in turn, opens the possibility to become aware of issues in one's interaction with work at an early time. Occupational neuroscience, in the future, might even hold a possibility for people to solve these issues far before they cause more obvious or even clinically relevant problems. A possible application could be the use of mobile devices measuring central variables related to effort investment. Utilizing methods such as functional near-infrared spectroscopy, a technique which measures the same changes as fMRI in a limited, yet mobile way, such devices could provide an on-line account of the acute effort investment, but also of the overall state of the user. Configuring these devices would probably still need to occur in stationary MRI machines, which provide qualitatively higher data for the whole brain. Yet, with the extensive data 
provided by MRI measurements, the devices could "learn" to deduce indicators for certain processes and states in their individual user from their own reduced data. Such devices could be connected to interfaces used for mental work, or for learning. Thereby, the workstation could actually help the user to pace his or her work, take breaks, eat, or to know when to engage in another type of activity. Such a setup would provide e.g. students with the chance to acquire the meta-skills necessary to utilize their full potential while safeguarding them against the pitfalls of maladaptive work patterns. Most of all, it would in our opinion be inevitable that students, but also workers who use this system, become more aware of their own state, and how the way they use their brain affects their experience beyond the immediate context of a task. In the end, such awareness could serve to empower people in any sort of mentally demanding context. With this awareness, they could meet the challenges posed on them by any occupation in an informed way, and utilize their full potential without unknowingly exceeding their limits. The research presented in this book forms merely the start of this process, yet it demonstrates that extending the reach of occupational psychology with modern neuroimaging methods potentially yields fundamental advances and applications in the future. 


\section{References}

Baumeister, R. F., Heatherton, T. F., \& Tice, D. M. (1994). Losing control. Academic Press San Diego, CA.

Bullmore, E., \& Sporns, O. (2012). The economy of brain network organization. Nature Reviews Neuroscience. doi:10.1038/nrn3214

Chandola, T., Britton, A., Brunner, E., Hemingway, H., Malik, M., Kumari, M., ... Marmot, M. (2008). Work stress and coronary heart disease: what are the mechanisms? European Heart Journal, 29(5), 640648. doi:10.1093/eurheartj/ehm584

Craig, A. D. (2009). How do you feel - now? The anterior insula and human awareness. Nature Reviews Neuroscience, 10(1), 59-70.

Damasio, A. (1999). The feeling of what happens. New York: Harcourt Brace \& Co.

Dosenbach, N. U. F., Fair, D. A., Cohen, A. L., Schlaggar, B. L., \& Petersen, S. E. (2008). A dual-networks architecture of top-down control. Trends in Cognitive Sciences, 12(3), 99-105. doi:10.1016/j.tics.2008.01.001

Duncan, J., \& Owen, A. M. (2000). Common regions of the human frontal lobe recruited by diverse cognitive demands. Trends in neurosciences.

Fair, D. A., Dosenbach, N. U. F., Church, J. A., Cohen, A. L., Brahmbhatt, S., Miezin, F. M., ... Schlaggar, B. L. (2007). Development of distinct control networks through segregation and integration. Proceedings of the National Academy of Sciences, 104(33), 13507.

Gailliot, M. T., Baumeister, R. F., DeWall, C. N., Maner, J. K., Plant, E. A., Tice, D. M., ... Schmeichel, B. J. (2007). Self-control relies on glucose as a limited energy source: Willpower is more than a metaphor. Journal of personality and social psychology, 92(2), 325.

Gray, M. A., Taggart, P., Sutton, P. M., Groves, D., Holdright, D. R., Bradbury, D., ... Critchley, H. D. (2007). A cortical potential reflecting cardiac function. Proceedings of the National Academy of Sciences, 104(16), 6818.

Hart, S. G., \& Staveland, L. E. (1988). Development of NASA-TLX (Task Load Index): Results of empirical and theoretical research. Human mental workload, 1, 139-183.

Juster, R.-P., McEwen, B. S., \& Lupien, S. J. (2010). Allostatic load biomarkers of chronic stress and impact on health and cognition. Neuroscience \& Biobehavioral Reviews, 35(1), 2-16. doi:10.1016/j.neubiorev.2009.10.002

Maguire, E. A., Woollett, K., \& Spiers, H. J. (2006). London taxi drivers and bus drivers: A structural MRI and neuropsychological analysis. Hippocampus, 16(12), 1091-1101. doi:10.1002/hipo.20233

McNay, E. C., Fries, T. M., \& Gold, P. E. (2000). Decreases in rat extracellular hippocampal glucose concentration associated with cognitive demand during a spatial task. Proceedings of the National Academy of Sciences, 97(6), 2881-2885. doi:10.1073/pnas.050583697

Modinos, G., Ormel, J., \& Aleman, A. (2009). Activation of Anterior Insula during Self-Reflection. (A. V. García, Ed.)PLoS ONE, 4(2), e4618. doi:10.1371/journal.pone.0004618

Mulder, G. (1980). The heart of mental effort. Unpublished doctoral dissertation, University of Groningen, Groningen, The Netherlands.

Mulder, G. (1986). The concept and measurement of mental effort. In G. R. J. Hockey, A. W. K. Gaillard, \& M. G. H. Coles (Eds.), Energetics and Human Information Processing. Dordrecht: Martinus Nijhoff.

Naccache, L., Dehaene, S., Cohen, L., Habert, M. O., Guichart-Gomez, E., Galanaud, D., \& Willer, J. C. (2005). Effortless control: executive attention and conscious feeling of mental effort are dissociable. Neuropsychologia, 43(9), 1318-1328.

Nelson, S. M., Dosenbach, N. U. F., Cohen, A. L., Wheeler, M. E., Schlaggar, B. L., \& Petersen, S. E. (2010). Role of the anterior insula in task-level control and focal attention. Brain Structure and Function, 214(5-6), 669-680. doi:10.1007/s00429-010-0260-2

Nickel, P., \& Nachreiner, F. (2003). Sensitivity and Diagnosticity of the 0.1-Hz Component of Heart Rate Variability as an Indicator of Mental Workload. Human Factors: The Journal of the Human Factors and Ergonomics Society, 45(4), 575-590. doi:10.1518/hfes.45.4.575.27094

Odle-Dusseau, H. N., Bradley, J. L., \& Pilcher, J. J. (2010). Subjective perceptions of the effects of sustained performance under sleep-deprivation conditions. Chronobiology International, 27(2), 318-333. doi:10.3109/07420520903502226 
Owen, A. M., McMillan, K. M., Laird, A. R., \& Bullmore, E. (2005). N-back working memory paradigm: A meta-analysis of normative functional neuroimaging studies. Human Brain Mapping, 25(1), 46-59. doi:10.1002/hbm.20131

Parasuraman, R. (2003). Neuroergonomics: Research and practice. Theoretical Issues in Ergonomics Science, 4(1-2), 5-20. doi:10.1080/14639220210199753

Peyron, R., Laurent, B., \& Garcia-Larrea, L. (2000). Functional imaging of brain responses to pain. A review and meta-analysis (2000). Neurophysiologie Clinique-Clinical Neurophysiology, 30(5), 263288.

Reid, G. B., Eggemeier, F. T., \& Shingledecker, C. A. (1982). Subjective workload assessment technique. DTIC Document.

Richter, M., \& Gendolla, G. H. E. (2007). Incentive value, unclear task difficulty, and cardiovascular reactivity in active coping. International Journal of Psychophysiology, 63(3), 294-301. doi:10.1016/j.ijpsycho.2006.12.002

Scholey, A. B., Harper, S., \& Kennedy, D. O. (2001). Cognitive demand and blood glucose. Physiology \& behavior, 73(4), 585-592.

Stam, C. J., \& van Straaten, E. C. W. (2012). The organization of physiological brain networks. Clinical Neurophysiology, 123(6), 1067-1087. doi:10.1016/j.clinph.2012.01.011

Van Dellen, E., de Witt Hamer, P. C., Douw, L., Klein, M., Heimans, J. J., Stam, C. J., ... Hillebrand, A. (2013). Connectivity in MEG resting-state networks increases after resective surgery for low-grade glioma and correlates with improved cognitive performance. NeuroImage: Clinical, 2, 1-7. doi:10.1016/j.nicl.2012.10.007

Van Den Heuvel, M. P., \& Hulshoff Pol, H. E. (2010). Exploring the brain network: a review on restingstate fMRI functional connectivity. European Neuropsychopharmacology, 20(8), 519-534.

Yeo, G. B., \& Neal, A. (2004). A Multilevel Analysis of Effort, Practice, and Performance: Effects; of Ability, Conscientiousness, and Goal Orientation. Journal of Applied Psychology, 89(2), 231-247.

Yeo, G. B., \& Neal, A. (2008). Subjective cognitive effort: A model of states, traits, and time. Journal of Applied Psychology, 93(3), 617.

Zijlstra, F. R. H. (1993). Efficiency in Work Behavior: A Design Approach for Modern Tools. Delft: Delft University Press. 


\section{Summary}

The concept of mental effort originates from the notion that humans have a limited capacity to perform mental tasks. More precisely, this concept describes the feeling of investing an abstract resource, a limited sort of "energy" into regulating oneself and one`s mental activities in order to perform a task (Zijlstra, 1993; Hockey, 1996).

The research that I performed in this project was aimed at identifying the neural correlates of mental effort investment; both in the sense of which brain regions take part in managing effort investment and in how the concept of a limited underlying resource relates to measurable parameters of neural functioning.

In the beginning, early psychological researchers focused mainly on the momentary limitations of the human information processing system. These limitations in processing capacity were readily observable in variables such as the time that a person needed to perform a given task, which increased after adding small subtasks to the main task. While observing and modeling the characteristics of the human information processing system at work, various researchers such as Kahneman (1973) or Moray (1968) noted that humans are able to regulate their processing of information in a flexible way.

This regulation, however, seems to consume a limited resource (Hockey, 1996). Sustained investment of this resource would lead to a reduction in its availability. More important, it was realized that the prolonged performance of tasks would also affect the general level of arousal of a person, and that maladjusted levels of arousal would require further investment of regulation in order to secure successful task performance. Mulder (1986) cast these two different taxing factors in his integrated model of mental effort: The need to invest regulative action to control one's information processing, as determined by the characteristics of the task and the baseline capacity of the individual, is in his model referred to as the task load. The need to invest regulative action to control one`s arousal state is conceptualized as a separate factor, the state load (Chapter 1 ).

The observation that the regulative capacity of an individual is limited has obvious implications: While humans can adapt their amount of effort investment in order to compensate for changes in task load or in state load, at some point the amount of effort that needs to be invested becomes higher than the amount of effort that the individual can or is willing to invest. Insufficient regulation eventually entails drops in performance. Such drops potentially have severe consequences, especially in occupational settings, where an individual can be in charge of machin- 
ery or vehicles. Hence, in particular occupational psychologists have sought ways to measure the amount of effort that an individual needs to invest into performing a task. Self-evaluations of effort investment with the help of rating scales have become an accepted technique in the field; with comparative research favoring scales which measure effort as one coherent concept, as opposed to multi-dimensional scales that attempt to measure different aspects of that experience of investing mental effort separately (Veltman \& Gaillard, 1996). Furthermore, different physiological variables such as parameters of the heart's electrical signals or such as blood glucose levels have been investigated as possible indicators of effort investment. More recently, also measurements of neural activity have been employed to identify indicators of mental effort investment (Chapter 2).

Currently available research into neural processes related to the investment of mental effort is frequently limited by the incomplete utilization of modern theoretical conceptualizations of mental effort. A neural correlate of mental effort investment has hence not been indicated to this day. As a result, subjective self-reports remain the most feasible measure of mental effort investment, with the limitation that it is not known to which processes in the human brain they are related. This lack of evidence precludes a conclusive validation of self-rating scales of mental effort and, to a degree, of the model that they are based on. More specific, self-rating scales rely on the assumption that acute experience and post-hoc evaluation of effort investment are identical. Furthermore, the integrated model of mental effort and as a result also one-dimensional self-report scales assume that task load and state load affect the same underlying resource, and in turn the experience of effort investment. Identifying neural correlates of mental effort investment is a necessary step to test these assumptions.

Equally important, a central assumption of the integrated model of mental effort, namely the assumption of a limited resource which fuels regulation, remains an abstract concept. Existing evidence clearly supports the concept of a limited resource. This concept is also a central element in current models in the closely related field of research into behavioral regulation, with a substantial body of convergent experimental evidence (Hagger, 2010). However, the current evidence only allows speculative interpretations of how the concept of a limited resource is related to neurophysiological processes in the human brain.

A better understanding of the neural correlates of mental effort is thus necessary for two reasons: First, to identify objective indicators of mental effort investment, second, to gain an idea of how the concept of a limited resource underlying all regulative processes is related to measurable parameters of neural functioning. Combining the conceptual expertise on the subject of mental effort that is present in occupational psychology with the methods offered by cognitive neuroscience offers a way to gain this knowledge (Chapter 3).

In chapter 4 a study (study 1 ) is described in which the neural correlates of the self-evaluation of mental effort investment were investigated. We based the experimental design on observations described by Nacchache et al. (2005) which suggest 
a dissociability of the neural correlates of evaluations of task difficulty and amount of invested effort by an individual. Participants performed several short blocks of a working memory task with three levels of difficulty. After each block, they indicated task difficulty and the amount of invested mental effort on two self-report scales. During task performance and during self-rating, brain activation was measured with fMRI. Results showed that a cluster in left anterior Insular Cortex (laIC) was selectively more active while participants performed self-ratings of effort investment. Additionally, the activity in laIC during task performance was modulated by changes in task demand in a similar way as the self-ratings of mental effort. We interpret these findings as evidence that self-evaluation of mental effort crucially relies on the integrative functions that have been argued (Craig, 2009) to take place in the anterior Insular Cortex. The observation of task-load-dependent activation changes in laIC during task performance suggests that laIC plays a role in the acute experience of effort investment, additional to its role in post-performance evaluation.

In chapter 5, I describe a study (study 2) that was designed to identify brain areas that react to both changes in task load and to changes in state load. This study builds on the design of study 1 . In study 1 , only manipulations of task load were implemented in the research design by incorporating different levels of difficult of the working memory task. In study 2 , we extended this design to also include variations of state load: Participants performed the same working memory task before and after either a low-effort free day or after an effortful work day. Again, effort investment was indicated by the participants after performing short blocks of the working memory task. The behavioral results showed an interaction between the effects of task load (the different levels of the task) and state load (induced by the two different day pre-treatments). Neuroimaging results showed that in particular activation in laIC during task performance shows the same interaction effect. This confirms the observation from study 1 that laIC plays an important in the acute experience of effort investment. Together with evidence from other studies which have demonstrated the functionality of laIC in a range of processes relevant to the investment of mental effort, this leads us to propose that laIC unifies several functions which serve to manage effortful behavior.

Chapter 6 describes the last of the three studies conducted in this research project. In this study (study 3), we employed measures of resting state functional connectivity to investigate how depleting the abstract energetical resource that is assumed in modern models of mental effort effects brain functioning. Measures of resting state brain activation were obtained with fMRI on a group of participants in the morning and in the afternoon, with either a free or a work day in between, identical to the state manipulation employed in study 2. Additionally, participants rated their level of subjective exhaustion and the amount of effort they perceived to be investing in a working memory task that they performed after each resting state measurement. In the afternoon, the day condition was shown to influence the behavioral measurement, manifested in increased reports of exhaustion and in- 
creased self-rated effort investment on an unchanged working memory task. More important, the day condition influenced the coherence in three functional networks, the executive network, the default mode network and the early visual network. In the early visual network and the default mode network, several local coherence scores correlated positively with exhaustion. We interpret these results as evidence that sustained functioning affects the functional connectivity between brain regions that cooperate in distributed networks.

In chapter 7, a brief overview of the three studies that I performed in the course of this project is given. I discuss the respective findings of each study, extending the discussions in the individual chapters. This is followed by a general discussion, in which I elaborate on the implications of my findings for the main questions of this project. The results from study 1 and 2 support the assumption that mental effort investment can be measured by a one-dimensional self-report scale, and that the acute experience and the post-performance self-rating are closely related.

The findings of study 3 give rise to a novel interpretation of the nature of the presumed resource underlying effortful regulation: I offer the view that instead of a limited energetical resource, it is rather the harmonic, flexible functional organization of different brain regions that is the key to effective volitional self-regulation of the individual. Sustained exerting of effortful regulation seems to transiently affect the ability of the brain to form some of the commonly reported functional networks in the same way as they are observed in un-exhausted participants. I discuss the implications of this view for the field of mental effort.

In the last part of chapter 7, I give an outlook of the possibilities that arise from the successful combination of occupational psychology and neuroimaging methods, and I outline a possible course for the emerging discipline of occupational neuroscience. 


\section{Samenvatting (Summary in Dutch)}

Het concept van mentale inspanning komt voort uit de observatie dat het menselijke vermogen om mentale taken uit te voeren beperkt is. Kort samengevat beschrijft dit concept het gevoel van het investeren van een beperkte, energieachtige bron ten behoeve van zelfregulatie. Deze is gericht op zelfregulatie van emotionele maar vooral ook van cognitieve mentale processen in het kader van de uitvoering van een taak (Zijlstra, 1993, Hockey; 1996).

Het door mij verrichte onderzoek had als doel de neurale correlaten van het investeren van mentale inspanning te identificeren. Dit onderzoek kende twee hoofdpunten: ten eerste, het lokaliseren van hersengebieden die het investeren van mentale inspanning reguleren en, ten tweede, hoe het concept van een onderliggende beperkte bron gerelateerd is aan meetbare parameters van hersenfunctie.

In hoofdstuk 1 beschrijf ik de historische oorsprong van het concept van mentale inspanning en de ontwikkeling van theoretische modellen. Vroege psychologische onderzoekers richtten zich vooral op acute beperkingen van het menselijke informatieverwerkingssysteem. Een voorbeeld van deze beperkingen was de toegevoegde tijd die een persoon nodig had na het uitbreiden van een taak. Later onderzoek verfijnde dit perspectief: onderzoekers zoals Kahneman (1973) of Moray (1968) lieten zien dat mensen hun informatieverwerkingssysteem op een flexibele manier kunnen reguleren. Deze regulatie lijkt dus een beperkte bron te verbruiken (Hockey, 1996). Het investeren van deze bron leidt op korte termijn tot een verminderde beschikbaarheid ervan. Bovendien bleek uit onderzoek dat langdurige mentale prestatie ook een effect had op de activatietoestand van het centrale zenuwstelsel. Indien de activatietoestand niet optimaal is, moet een persoon eerst regulatie toepassen om een activatietoestand te bereiken die prestatie mogelijk maakt voordat de taak uitgevoerd kan worden.

Mulder (1986) gebruikte deze tweedeling van factoren als basis voor zijn Integrated model of Mental Effort. Alle noodzaak voor regulatie van informatieverwerking zoals bepaald door de eisen van de taak wordt in de zgn. task load samengevat. De noodzaak om de activatietoestand te reguleren om überhaupt in staat te zijn een mentale taak uit te voeren wordt state load genoemd. Samen vormen task load en state load de zgn. mental work load (hoofdstuk 1).

De observatie dat het menselijk vermogen om informatie te verwerken beperkt is heeft een aantal implicaties. Mensen zijn in staat om door verhoging van hun investering van mentale inspanning verhogingen in task- en state load te compenseren maar de mogelijkheid om meer inspanning te leveren is beperkt. Als de com- 
binatie van task- en state load meer regulatie (en dus inspanning) vergt dan een persoon kan of wil leveren, zal de taakprestatie dalen. Zulke dalingen van prestatie kunnen dramatische gevolgen hebben. Vooral in de context van arbeid, waar mensen verantwoordelijkheid voor machines of voertuigen dragen, zijn de gevolgen van prestatiedaling niet te onderschatten. Het is daarom niet verwonderlijk dat vooral arbeidspsychologen naar mogelijkheden hebben gezocht om de benodigde mentale inspanning die een persoon op een gegeven moment moet leveren te meten. Zelfevaluaties van de hoeveelheid geïnvesteerde mentale inspanning met behulp van schalen zijn inmiddels een geaccepteerde methode. Onderzoek wijst uit dat schalen die de hoeveelheid inspanning als een enkele dimensie meten betere resultaten leveren dan schalen die verschillende aspecten van het leveren van inspanning apart te meten (Veltman \& Gaillard, 1996). Daarnaast is ook onderzoek verricht naar de bruikbaarheid van fysiologische variabelen zoals bloedsuiker of hartstromen als indicatoren van geleverde mentale inspanning. Recent werden niet alleen perifere maar ook centrale variabelen als mogelijke indicatoren onderzocht. (hoofdstuk 2).

Onderzoek naar de onderliggende neurale processen die betrokken zijn bij het leveren van mentale inspanning maakt onvoldoende gebruik van moderne theoretische concepten en modellen van mentale inspanning. Als gevolg hiervan is dus tot op heden geen echt neuraal correlaat van mentale inspanning gerapporteerd. Subjectieve zelfevaluatie met behulp van schalen vormt nog steeds de meest accurate manier om mentale inspanning in de praktijk te meten. De neurale mechanismen die leiden tot een indicatie op een dergelijke schaal zijn niet bekend. Het ontbreken van vooral deze informatie bemoeilijkt het toetsen van meetmethodes en de onderliggende theoretische assumpties over mentale inspanning,. Alle zelfevaluatieschalen hanteren bijvoorbeeld de assumptie dat de door een persoon herinnerde investering van mentale inspanning na het uitvoeren van een taak overeen komt met de daadwerkelijk ervaren hoeveelheid tijdens de uitvoering. Het integrated model of mental effort (Mulder,1986) neemt verder aan dat task- en state load dezelfde onderliggende beperkte bron gebruiken. Deze aanname is tevens cruciaal voor alle eendimensionale schalen voor mentale inspanning. De identificatie van neurale correlaten van het investeren van mentale inspanning is een nodige stap om deze assumpties te toetsen. Een andere cruciale assumptie van het integrated model of mental effort is dat regulatie door een beperkte bron aangedreven wordt. Cognitief onderzoek, ook uit het gerelateerde veld van gedragsregulatie, steunt deze aanname (Hagger, 2010). De bevindingen uit onderzoek beperken interpretaties over de relatie van het concept van een beperkte bron en het functioneren van het menselijk brein tot speculaties.

Een beter begrip van neurale correlaten van het investeren van mentale inspanning is dus om twee redenen noodzakelijk: ten eerste, om een objectieve indicator voor de hoeveelheid geïnvesteerde mentale inspanning te vinden, en ten tweede, om beter te begrijpen hoe het theoretisch concept van een beperkte bron gerelateerd is aan neurofysiologische processen. Hiervoor is het belangrijk om de 
uitgebreide theoretische en conceptuele kennis vanuit de arbeidspsychologie te verbinden met de technieken waarover het veld der cognitieve neurowetenschappen beschikt (hoofdstuk 3).

In hoofdstuk 4 beschrijf ik een onderzoek (studie 1) waarin ik de hersenactivatie van proefpersonen in kaart breng tijdens het invullen van eendimensionale schalen, om de geïnvesteerde hoeveelheid mentale inspanning te meten. Het doel van dit onderzoek was om de neurale activatie gerelateerd aan zelfevaluatie van geïnvesteerde mentale inspanning te scheiden van activatie tijdens een evaluatie van taakmoeilijkheid. Eerder onderzoek heeft uitgewezen dat deze twee op elkaar lijkende evaluaties van verschillende neurale structuren afhangen (Nacchache et al., 2005). In mijn onderzoek voerden de deelnemers enkele korte blokken van een geheugentaak uit. De blokken hadden drie verschillende niveaus van moeilijkheid. $\mathrm{Na}$ ieder blok gaven de deelnemers op een eendimensionale schaal aan hoeveel mentale inspanning ze geïnvesteerd hadden en hoe hoog ze de moeilijkheid van de taak schatten. Tegelijkertijd werd de hersenactivatie van de deelnemers met behulp van functionele magnetresonantietiomografie (fMRT) gemeten. De resultaten wezen uit dat vooral een gebied in het linker anteriore insulaire cortex (laIC) sterker geactiveerd was tijdens het beoordelen van geïnvesteerde mentale inspanning. Deze bevinding wijst volgens ons uit dat het beoordelen van eerder geleverde mentale inspanning heel sterk op het integreren van verschillende zelf-gerelateerde informatiestromen aangewezen is. Deze vindt volgens Craig (2009) vooral in het voorste gedeelte van de insulaire cortex plaats vindt. Bovendien wijst een activatieverschil in laIC overeenkomend met de verschillen in taakmoeilijkheid tijdens de uitgevoerde werkgeheugentaak op een mogelijke rol van laIC bij de ervaring van het investeren van mentale inspanning.

In hoofdstuk 5 wordt een onderzoek (studie 2) beschreven dat op de resultaten van studie 1 voortbouwt. Studie 2 had als doel om hersengebieden te identificeren die op veranderingen in zowel de task load als de state load reageren. Terwijl in studie 1 alleen manipulaties van de task load gebruikt werden, maakte in studie 2 ook een manipulatie van state load een deel uit van het experimentele paradigma. Hiervoor gebruikten we een soortgelijke werkgeheugentaak als in studie 1, maar nu lieten we de proefpersonen deze ofwel voor en na een inspannende dag, ofwel voor en na een vrije dag uitvoeren. Wederom beoordeelden de deelnemers na ieder kort blok van de taak de hoeveelheid geleverde mentale inspanning. De gedragsdata wezen uit dat er een interactie-effect tussen de manipulatie van task- en van state load aanwezig was. Het effect van de verschillende levels van taakmoeilijkheid (task load) op het ervaren niveau van mentale inspanning verschilde dus naarmate de proefpersonen een werk- of een vrije dag doorgebracht hadden (hierdoor werd een verschil in state load opgewekt). De analyse van de fMRT - data gemeten tijdens de taakuitvoering wezen op hetzelfde interactie-effect uitsluitend in de eerder gevonden laIC. Hieruit interpreteren wij dat laIC al tijdens de taakuitvoering een belangrijke rol voor het management van mentale inspanning speelt. 
In hoofdstuk 6 beschrijf ik een derde onderzoek (studie 3). De focus lag hierbij op het onderzoeken van de verandering van meetbare parameters van hersenfunctie door langdurige prestatie. In dit onderzoek heb ik dezelfde opzet gebruikt als in studie 2, om wederom een paradigma met zowel manipulaties van task- en state load te creëren. Naast de mentale inspanning werd in dit experiment ook het vermoeidheidsniveau van de proefpersonen gemeten. De fMRT - meting van dit experiment was op een periode van rust gefocust waarin de deelnemers juist niet met een taak bezig waren: het zogenoemde Resting-State-paradigma. Zoals verwacht gaven de deelnemers aan dat de werkgeheugentaak na een veeleisende werkdag meer inspanning vereiste dan na een vrije dag. Tevens waren de deelnemers na een werkdag vermoeider. De dagconditie bleek bovendien een duidelijke invloed te hebben op een aantal bekende functionele netwerken. Maten van coherentie in sommige knooppunten van enkele netwerken vertoonden zelfs een significante correlatie met de gerapporteerde maten van vermoeidheid. Hieruit concluderen wij dat langdurige investering van mentale inspanning een temporeel effect kan hebben op de functionele netwerken van het menselijk brein.

Hoofdstuk 7 begint met een overzicht van de verrichtte onderzoeken. Ik bespreek hier nogmaals de belangrijkste bevindingen uit elk apart onderzoek en breid de discussie van de verschillende hoofdstukken uit. Vervolgens bespreek ik de resultaten in het kader van de theoretische achtergronden en van de beginvragen die mij tot dit onderzoek aan hebben gezet. De resultaten van studie 1 en 2 ondersteunen de assumpties die vooral aan eendimensionale schalen ten grond liggen, namelijk dat het acute ervaren van mentale inspanning en het latere evalueren ervan nauw samenhangen.

Het hersengebied dat in studie 1 actiever was tijdens het evalueren van mentale inspanning bleek in studie 2 beïnvloed te worden door zowel variaties in task load als in state load tijdens het uitvoeren van een taak. Deze gezamenlijke invloed van task- en state load op hersenactivatie wijst uit dat eendimensionale schalen inderdaad een goed instrument zijn voor het meten van mentale inspanning. De bevindingen van studie 3 zetten aan tot een nieuwe interpretatie van de veronderstelde energieachtige aard van de onderliggende bron: Ik bespreek een zienswijze waarin ik voorstel dat de onderliggende bron, welke door zelfregulatie verbruikt wordt, niet zo zeer als letterlijke energie dient te worden beschouwd, maar als het vermogen van het menselijke brein om flexibele, doelgerichte en gebalanceerde functionele netwerken uit verschillende hersengebieden te vormen. Dit vermogen is uitermate belangrijk voor een effectieve regulatie van mentale processen. Mijn resultaten suggereren dat dit vermogen door langdurige inspanning tijdelijk beïnvloed kan worden. In het laatste stuk bespreek ik de implicaties van mijn onderzoek, ook met betrekking tot de mogelijkheden die door het succesvol combineren van arbeidspsychologie en neuro-wetenschappelijke methodes ontstaan. Ik sluit af met een visie op een mogelijke koers voor het jonge veld van occupational neuroscience. 


\section{Acknowledgements}

I have looked forward to write these lines for a number of years now. Finishing this book in the early hours of morning resembles all the times that I have been writing papers, analyzing data, programming some gadget, or doing anything else from the myriad of things in research that generally need to be done right now. The future holds more of this, for sure, and I am happy to have reached this point. I could not have done this without the help of a great number of people. I would like to thank them.

First, I would like to thank my parents. From an early age on they have nourished my curiosity, so it could outweigh my fears, and I would not have been able to make this journey without this. My parents have always supported me unconditionally with everything they had and gave me the freedom to find my own way. I am grateful to have you.

Alex, my lover, my friend. You were always there for me when I was feeling like the most stupid person to have ever walked the floors of the university. You have helped me to assume myself by never stopping to believe in me. I thank you for all the hours that I could discuss my ideas with you. By sharing your giftedness in statistics with me, you have helped me to actually enjoy the statistical way of thinking. You are the ultimate proof that nerds can be sexy.

Fred and Rainer, I cannot thank you enough. You have opened a door for me. You gave me the freedom to discover science in my own way. You even gave me the space to make mistakes, and you helped me when I did. Next to all the scientific and academic knowledge that you have shared with me, there are two things that I especially enjoyed: Fred, your respectful style of leading people is truly ingenious. The few things I have picked up from this so far have helped me tremendously in situations where I had to lead others. I am glad that I can learn this from you. Rainer, your way of focusing on positive aspects and sharing this view with a passion is contagious. This is a beautiful way of making people grow.

Kierty - you have taught me so much. About martial arts. About thinking. About myself. About the world. Thank you. 
Alice and Alicia, my paranymphs. You have shared a lot of the way with me. Thank you for always being there for me, for listening to my whining and for helping me with all the things I would forget with my airhead.

Mariella - next to being the administrative equivalent of wonder woman, thank you for all the help with getting a million things sorted out (which according to you is "just your job" - I beg to differ!)

Astrid and Nicolette - You have helped me to see the light in my research so many times! Thank you for all the pep-talks that you gave me when I thought again that I was the most stupid person to ever walk around in this university. Thanks to you, I could be as stupid as I want, but I would never be alone.

Alex (de Voogt) - thank you for showing me different perspectives. On research, on food, on life! You showed me to think in non-linear ways, to be observant and to make a choice at the right time. Although some of these choices are costly (especially the three-starred ones), I have never regret a single one!

Armin, Hester and the rest of the Brain Innovation team - I got myself into huge trouble several times by assuming that no matter how naive I plan my experiments, there would be a way to analyze it with BrainVoyager. But you actually made this happen. Every time. Even when it involved dealing with the output of outrageously programmed third-party software. Even if you had to actually sit down with me and program a fix. You people are amazing!

Sven and Lotti - You always had my back in the MRI lab. Whatever happened, I knew I could count on you.

My colleagues at Work and Social Psychology - You welcomed me in your group, and you gave me the chance to learn a completely different field of psychology then I had known before.

Michelle and Martin - Your help during the last study of this project was a crucial factor that made this such a success. Also, doing research together with you was so much more enjoyable. Seeing that you are by now conducting your own PhD research makes me very happy.

Maaike - for all your wisdom during this time and for letting me use your work as cover art. I could not have pictured a better look for the book!

Björn, Steve, Chris, Jesse, Joel, Marit and the rest of the gang - I am very, very happy to have you as my friends. Without your support, these last years would have been so much harder. Every time I look at the group picture we took before I went to Hamburg, I feel so warm! 


\section{Curriculum vitae}

Tobias Otto was born in 1981 in Germany. He obtained his Abitur at the HugoJunkers-Gymnasium in Mönchengladbach, Germany in 2000. After completing his mandatory government service and taking a short excursion into the field of business studies at Fontys Hogeschool in Venlo, the Netherlands, he began his study of Psychology at Maastricht University, the Netherlands in 2002. After completing his Bachelor of Science in Biological Psychology in 2005, Tobias was given the opportunity to be among the first to join the newly created Research Master program at the Faculty of Psychology of Maastricht University. This program gave him the chance to conduct his first own fMRI research under the supervision of Prof. Dr. Rainer Goebel and Dr Alard Roebroeck. Specializing in cognitive neuroscience, Tobias graduated as a Master of Science in 2007. Directly afterwards, he started a PhD project under the supervision of Prof. Dr. F.R.H. Zijlstra and Prof. Dr. Rainer Goebel, the results of which are described in this book. After a Post-doctoral fellowship at Universitätsklinikum Schleswig-Holstein, Klinik für Neurologie in Lübeck, Germany from mid-2012 to mid-2013, Tobias returned to Maastricht University in September 2013. He is currently employed there as Assistant Professor of Occupational Neuroscience. 